\title{
Algebraic Approach to Interacting Quantum Systems
}

\author{
C.D. Batista and G. Ortiz \\ Theoretical Division, Los Alamos National Laboratory, Los Alamos, NM 87545
}

(Dated: Received October 22, 2018)

\begin{abstract}
We present an algebraic framework for interacting extended quantum systems to study complex phenomena characterized by the coexistence and competition of different states of matter. We start by showing how to connect different (spin-particle-gauge) languages by means of exact mappings (isomorphisms) that we name dictionaries and prove a fundamental theorem establishing when two arbitrary languages can be connected. These mappings serve to unravel symmetries which are hidden in one representation but become manifest in another. In addition, we establish a formal link between seemingly unrelated physical phenomena by changing the language of our model description. This link leads to the idea of universality or equivalence. Moreover, we introduce the novel concept of emergent symmetry as another symmetry guiding principle. By introducing the notion of hierarchical languages, we determine the quantum phase diagram of lattice models (previously unsolved) and unveil hidden order parameters to explore new states of matter. Hierarchical languages also constitute an essential tool to provide a unified description of phases which compete and coexist. Overall, our framework provides a simple and systematic methodology to predict and discover new kinds of orders. Another aspect exploited by the present formalism is the relation between condensed matter and lattice gauge theories through quantum link models. We conclude discussing applications of these dictionaries to the area of quantum information and computation with emphasis in building new models of computation and quantum programming languages.
\end{abstract}

PACS numbers: 03.65.Fd, 05.70.Fh, 75.10.Jm, 71.10.-w

\section{INTRODUCTION}

Unveiling the fundamental principles behind complex behavior in matter is a problem at the frontiers of condensed matter physics and embraces cases like the high-temperature superconductors, heavy fermions, and low-dimensional electron liquids. Generically, describing the structure and behavior of matter involves studying systems of interacting quantum constituents (bosons, fermions, spins, gauge fields) whose fields satisfy the basic laws of quantum mechanics. Nevertheless, the plethora of complex phenomena exhibited by nature exceeds our ability to explain them, in part, because the whole is not necessarily the sum of its parts [1] and thus typical perturbation-like-theory arguments or standard mathematical techniques are not appropriate to disentangle its mysteries. In this paper we present a unifying algebraic framework for interacting extended quantum systems that enables one to study complex phenomena characterized by the coexistence and competition of various states of matter.

The emergence of such complex phenomena may be the result of very simple, undiscovered, principles that conspire against any straightforward explanation. Nonetheless, we expect that two pillars of modern science, symmetry and topology, are key guiding principles behind those fundamental laws of emergence. Group theory and geometry have been fundamental to the physics of the twentieth century and we count on them to continue playing such a role. Indeed, the notion of symmetry and its breakings have shaped our current conception of nature. Understanding the idea of invariance and its corresponding conservation laws turns out to be as fundamental as determining the causes that prevent such harmony and leads to more complex behavior. Other kinds of order, not described by broken symmetries, are possible. For example, quantum orders of topological nature distinguish the internal structure of the state without breaking any local symmetry, and its study is beyond the scope of the present paper [2]. Another example is provided by the notion of emergent symmetries where, as the name indicates, the number of symmetries of the state increases as the temperature is lowered, contrary to the broken symmetry case. These concepts are at the heart of the field of quantum phase transitions that studies the changes that may occur in the macroscopic properties of matter at zero temperature (i.e., $T=0$ ) due to changes in the parameters characterizing the system.

In this regard, the development of exact algebraic methods is one of the most elegant and promising tools toward the complete understanding of quantum phases of matter and their corresponding phase transitions. Typically, there are no distinct length or time scales which separates the different competing orders and often these systems are near quantum criticality which makes their study extremely complicated, if not impossible, by the traditional techniques, such as standard meanfield or perturbation theories. The reason which prevents the effective use of these theories is precisely the key for the successful application of the algebraic methods, namely, the absence of a small parameter for the various complex quantum orderings. In other words, one cannot systematically apply renormalization group ideas and easily integrate out irrelevant degrees of freedom. On the other hand, those competing orders are frequently related by symmetry principles characterizing the critical behavior caused by the competing interactions, thus increasing the symmetry group of the effective low-energy physics.

In the present manuscript we will be concerned with quantum lattice systems. A quantum lattice is a composite system identified with $\mathbb{Z}^{N_{s}}$ where $N_{s}$ is the total number of lattice sites, and associated to each lattice site (or mode) $\mathbf{i} \in \mathbb{Z}^{N_{s}}$ is a Hilbert space $\mathcal{H}_{\mathbf{i}}$ of finite dimension $D$ describing the local modes. The total Hilbert space, i.e., state space of the lat- 
tice, is the tensor product $\otimes$ of the local modes state spaces, $\mathcal{H}=\bigotimes_{\mathbf{i}} \mathcal{H}_{\mathbf{i}}$, in the case of distinguishable subsystems. (Notice that $\mathcal{H}$ may support inequivalent tensor product decompositions.) Indistinguishability places an additional constraint on the space of admissible states which manifests itself in their symmetry properties (e.g., antisymmetric states in the case of fermions) and, consequently, the physical Hilbert space $\mathcal{H}$ is a subspace of $\bigotimes_{\mathbf{i}} \mathcal{H}_{\mathbf{i}}$. A pure state of the system (at $T=0$ ) is simply a vector $|\Psi\rangle$ in $\mathcal{H}$, and an observable is a selfadjoint operator $\hat{\mathcal{O}}: \mathcal{H} \rightarrow \mathcal{H}$. The dynamical evolution of the system is determined by its Hamiltonian $H$. The topology of the lattice, determined by the connectivity of the graph induced by the interactions in $H$, is an important element in establishing complexity. Unless specified, we will always consider regular lattices of coordination $z$. In the case of quantum continuous systems we can still use the present formalism after discretizing, for example, the space coordinates. Going beyond this approach is deferred for a possible later publication.

Every given physical system is represented by a language which consists of a set of operators that acts irreducibly on the Hilbert space $\mathcal{H}$. For example, if our system consists of a collection of interacting electrons a natural language is a set of spin 1/2 fermion field operators. Defining a language amounts to establishing the state space $\mathcal{H}$ used to describe the physical system, while a Hamiltonian written in terms of that language comes to setting up the quantum dynamics expected to depict the phenomenon of interest. Can we connect the different (spin-particle-gauge) languages of nature within a single algebraic framework? The answer to this question is yes and the key outcome is a set of dictionaries relating the languages representing the different physical systems. More precisely, by dictionary we mean an isomorphic (one-to-one) mapping connecting two languages. The existence of dictionaries provides not only a tool to explore complexity but leads naturally to the fundamental concept of universality, or equivalence, meaning that different physical systems display the same behavior. The concepts of language and dictionary are introduced in section III Previous to our work there were two seemingly unrelated examples of these types of mappings: The Jordan-Wigner (1928) [3] and MatsubaraMatsuda (1956) [4] transformations. We have not only generalized these $(S U(2))$ transformations to any spin, spatial dimension and particle statistics but have proved a fundamental theorem that permits the connection of the generators of the different languages which can be used to describe a given physical problem.

Section凹starts defining the concept of a bosonic language. Then we prove our fundamental theorem and establish the necessary conditions for application of a given bosonic language to describe the physical system under consideration. The main result of this section is a proof of the existence of mappings between the generators of any pair of languages which belong to the same class, i.e., that can be used to describe the same physical system. The necessary and sufficient condition for any pair of languages to be connected is that the dimension of their local Hilbert spaces $D$ be the same. In addition we prove that for each class of languages, there is at least one that is realized by the generators of a Lie algebra. To complete this picture, we introduce the notion of hierarchical language and prove that there is at least one hierarchical language in each class. Those languages are, in general, the most convenient ones to characterize the different quantum phases of the system under consideration.

In section III we extend these conclusions to non-bosonic systems by introducing operators which transmute the modes statistics. These operators have a local and a non-local component. The non-local part is a trivial generalization of the transformation introduced by Jordan and Wigner [3] to map spins $S=1 / 2$ into spinless fermions. The local component transmutes the statistics associated to the interchange of particles which are sharing the same lattice site i. Adding the transmutation of statistics to the fundamental theorem completes the characterization of each class of equivalent languages. In other words, the classes of bosonic languages defined in section $\amalg$ are expanded in section III to include fermionic, anyonic, or hybrid (para-) languages.

The most natural choice of local Hilbert space $\mathcal{H}_{\mathbf{i}}$ is the one spanned by a single site (or mode) $\mathbf{i}$ basis of dimension $D$. The possibility to decompose the total Hilbert space of the problem $\mathcal{H}$ into subspaces or subsystems which are not necessarily single sites opens up the possibility to generate other dictionaries with unforeseen applications. (Remember that $\mathcal{H}$ may support different tensor product decompositions.) Section [V] expands on this concept and show the simple case of a dictionary that uses as local Hilbert space a bond $(\mathbf{i}, \mathbf{j})$ state space $\mathcal{H}_{\mathbf{i}} \otimes \mathcal{H}_{\mathbf{j}}=\mathcal{H}_{\mathrm{ij}}$ of dimension $D^{2}\left(\mathcal{H}=\bigotimes_{(\mathbf{i}, \mathbf{j})} \mathcal{H}_{\mathrm{ij}}\right)$, and which is mapped onto a site Hilbert space of the same dimension.

Section $\mathrm{V}$ is devoted to show explicit connections between equivalent languages. The main purpose of this section is to illustrate through examples the application of the dictionaries developed in the previous two sections. Out of the many possible transformations predicted by our fundamental theorem and the transmutation of statistics, we selected a few of them which are useful for the applications described in the later sections. However, it is important to note that the procedure described to find the mapping connecting two languages in the same class is very straightforward. In addition, we show that the fractional exclusion statistics algebras emerge naturally from the present formalism.

Given a model Hamiltonian operator representing a quantum system, we can use our dictionaries to translate it into another equivalent Hamiltonian written in a different language. In other words, we can write down the same Hamiltonian operator in many distinct ways according to the different languages that are included in the corresponding class. Since some of these languages are naturally associated to specific physical entities (like spins, particles, gauge fields, etc.), the corresponding translation provides a rigorous connection between seemingly unrelated physical systems. This is the main subject of section VI where we show examples of completely different quantum lattice systems described by the same model. Another interesting aspect of these mappings is the potential to unveil hidden symmetries of the Hamiltonian, possibly leading to exact or quasi-exact solutions [5]. Sometimes particular languages allow us to recognize invari- 
ant subspaces of our Hamiltonian. When the action of our Hamiltonian is restricted to an invariant subspace, the corresponding operator can always be written in a language which is more elementary than the original one (i.e., it realizes a reduced number of degrees of freedom). In some cases, this procedure enables one to recognize the hidden symmetries which lead to the quasi-exact solution of the model considered (i.e., the model is integrable within the invariant subspace). The quasi-exact solution of the one-dimensional $t-J_{z}[6]$ model belongs to this class of problems. It is important to remark that this quasi-exact solution leads to the exact determination of the quantum phase diagram and the charge excitations of the $t-J_{z}$ model.

A new notion beyond Landau's concept of broken symmetry [7, 8] is the complementary idea of emergent symmetry, i.e., the fact that new symmetries not realized in the Hamiltonian describing the system can emerge at low energies. There are many instances in which the high-symmetry, low-energy, effective theory can be derived in an exact way, i.e., without appealing to approximate schemes like the renormalization group. For instance, the exact effective theory (ground state plus any charge excitations) of the one-dimensional $t$ $J_{z}$ model is the $S=1 / 2 X X Z$ model [6]. It is well known that the $X X Z$ model has an infinite number of symmetries which make it exactly solvable by the Bethe ansatz. Another example is provided by the family of spin Hamiltonians for which the ground state is a product of spin singlets. This family includes the Majumdar-Ghosh [9] model and many other generalizations [9, 10, 11, 12, 13]. These and other simple examples introduced in section VII illustrate the fundamental concept of emergent symmetry which, as we will show, can be used as a guiding principle to find new states of matter. In a sense that will become clear later, this concept not only formalizes but also provides a systematics to the principle of adiabatic continuity that P.W. Anderson [8] has advocated as one of the two most important principles of condensed matter physics (together with the concept of broken symmetry). States like the Fermi liquid or the band insulator which do not correspond to any broken symmetry can be characterized by their corresponding emergent symmetries. In this way one can establish a formal connection between different phases. For instance, the emergent symmetry of the band insulator is analog to the emergent symmetry of the singlet dimer magnetic states. In most cases, the notion of emergent symmetry is approximate; however, still provides a guiding principle to identify the relevant degrees of freedom, and the nature of the ground state and low-energy excitations.

Coexistence and competition of different quantum orderings associated to broken continuous symmetries is one of the main subjects of section VIII There we show the fundamental role played by the hierarchical languages for the classification of the possible order parameters and the calculation of the quantum phase diagram of a given model. We note that the hierarchical languages are the most natural ones to provide a unified description of the order parameters characterizing each phase. To illustrate the procedure we consider the example of the Heisenberg $S U(N)$ Hamiltonians written in different languages. We then take the local $S U(N)$ order parameter and reduce its components according to the different subgroups of $S U(N)$ that can be used to generate an equivalent language (for instance $S U(2)$ ). As an illustration, we show that the local order parameter for an $S=1 S U(2)$-spin Hamiltonian can be either the usual magnetization or a spin-nematic order parameter. To conclude this section, we show how to obtain the quantum phase diagram of the bilinear-biquadratic $S=1$ Heisenberg model (for spatial dimensions $d>1$ ) just by writing the Hamiltonian in the $S U(3)$ hierarchical language. It is important to remark that this zero temperature phase diagram was only known for semi-classical spins [14]. The fact that there is no calculation involved in the elaboration of this quantum phase diagram permits the reader to appreciate the power and potential applications of this algebraic framework.

The formal connection between lattice models in condensed matter physics and lattice gauge theories of highenergy physics is described in section $\amalg$ There we take advantage of the existing quantum link models and the connection between spins and other degrees of freedom, such as gauge fields, which emerges from our algebraic approach.

In addition to the fascinating field of quantum phase transitions and statistical mechanics, our algebraic approach can be applied to the field of quantum information [15]. This new paradigm of information processing takes advantage of the fundamental laws of quantum mechanics to perform operations which can be done at least as efficiently (i.e., with polynomial complexity) as with classical devices. The device that performs the manipulation of information is named quantum computer and the basic unit of information is the qubit (i.e., a two-level system). The quantum computer (a quantum many-body system in disguise) consists of an isolated set of quantum degrees of freedom which can be controlled and manipulated at the quantum level to perform the required operations. The different languages associated with the description of these quantum degrees of freedom are possible programming languages and indeed may realize different models of quantum computation. The development of dictionaries connecting these languages is relevant not only to improve the efficiency of a quantum computer, but also to model and simulate different physical phenomena. The applications of our algebraic framework to the fields of quantum information and quantum computing are discussed in section $\mathrm{X}$ There we show how each physical realization of a quantum computer has a class of languages associated to the realization of the quantum operations. For instance, if we consider the typical case of interacting $S=1 / 2$ spins the most natural language is the one generated by the Pauli matrices. Hence we can use the dictionary provided by the Jordan-Wigner (JW) [3] transformation to simulate a fermionic system [16]. In this regard, the purpose of this paper is to generalize this idea to other possible realizations of quantum computers. We also discuss the potential applications of this algebraic framework to the description of recent experiments on bosonic systems in optical lattices.

Finally, in section XI we summarize the main concepts introduced in this paper. 


\section{GENERAL LIE ALGEBRAIC FRAMEWORK}

The notions of Hilbert space and linear maps or operators are central to the formulation of quantum mechanics [17]. Pure states (wave functions) are elements of a Hilbert space and physical observables are associated to Hermitian (selfadjoint) operators which act on that space, and whose eigenvalues are selected by a measuring apparatus. The role of linear operators in quantum mechanics is not restricted to the representation of physical observables. Non-Hermitian operators are very often used. That is the case, for instance, of the creation and annihilation operators in second quantization. Moreover, the unitary operator that describes the time evolution of the system is in general non-Hermitian. These observations simply tell us that linear operators in general (Hermitian and non-Hermitian) play a more general role in quantum mechanics since they provide the mathematical language which is required to describe the dynamical behavior of our quantum system.

What do we mean by the term mathematical language? In order to provide a rigorous answer for this question it is important to first determine what is the mathematical structure associated to the set of quantum operators. Quantum operators form a complex linear (vector) space under the sum and the multiplication by a scalar over the field of complex numbers. If we augment this vector space with a bilinear operation (product $\square$ between two operators), the set form an $a l$ gebra. Quantum mechanics also requires this operation being non-commutative and associative. This additional structure makes the set of quantum operators form an associative algebra. In principle, any operator in this algebra can play a role in the description of our quantum system. However, one can always select a subset (which forms a basis) in such a way that any quantum operator can be expressed as a function of the selected ones. For instance, we can use the Pauli matrices $\left\{\sigma^{x}, \sigma^{y}, \sigma^{z}\right\}$

$$
\sigma^{x}=\left(\begin{array}{ll}
0 & 1 \\
1 & 0
\end{array}\right), \sigma^{y}=\left(\begin{array}{cc}
0 & -i \\
i & 0
\end{array}\right), \sigma^{z}=\left(\begin{array}{cc}
1 & 0 \\
0 & -1
\end{array}\right),
$$

to express any quantum operator associated with a spin $1 / 2$. The particular subset of operators that we choose to express any quantum operator is the mathematical language that we will use for the quantum description of our system. The elements of this subset will be called generators of the language.

What are the conditions a given set of quantum operators must satisfy to become a language? How many different languages can be used to describe a quantum system? What is the connection between the different languages? What is the most appropriate language to describe a particular system? What is the relation between language and symmetry generators? How can languages help us understand the phenomenon of universality? (It turns out that the notions of language and universality are closely related whenever a common language can be used to describe seemingly unrelated physical phenomena.) A great part of this paper is dedicated to answer these questions. In particular, in this section we introduce the notion of bosonic language, we establish a formal connection or dictionary between the different bosonic languages associated with a given bosonic system, and we also establish a formal relation between bosonic languages and Lie algebras.

One of the fundamental steps toward a deeper understanding of classical systems was the recognition that dynamical variables which define the phase space are generators of continuous transformations. The set of continuous transformations forms a group and the infinitesimal generators provide a basis for a Lie algebra which is related to the group. A group is a non-empty set which is closed under an associative product $\square$, it contains an identity, $\mathbf{1}$, and all of its elements are invertible. When the transformations in the group leave the equations of motion invariant the set becomes a symmetry group for the considered system. A real (complex) Lie algebra $\mathcal{L}$ is a linear space over the field $F$ of real (complex) numbers which is closed under a non-associative Lie product $[$,$] that$ satisfies $(\mathfrak{a}, \mathfrak{b}, \mathfrak{c} \in \mathcal{L}$ and $\alpha, \beta \in F)$ :

$$
\begin{aligned}
{[\alpha \mathfrak{a}+\beta \mathfrak{b}, \mathfrak{c}] } & =\alpha[\mathfrak{a}, \mathfrak{c}]+\beta[\mathfrak{b}, \mathfrak{c}] \\
{[\mathfrak{a}, \mathfrak{b}] } & =-[\mathfrak{b}, \mathfrak{a}] \\
0 & =[\mathfrak{a},[\mathfrak{b}, \mathfrak{c}]]+[\mathfrak{b},[\mathfrak{c}, \mathfrak{a}]]+[\mathfrak{c},[\mathfrak{a}, \mathfrak{b}]]
\end{aligned}
$$

Whenever a continuous transformation is a symmetry of our physical system, the infinitesimal generator, which is an element of the corresponding Lie algebra, becomes a conserved quantity. As a consequence, it is convenient to choose that quantity as one of the coordinates for the phase space of our system.

In the same way, it may be appropriate to choose the quantum operators (generators of the language) for the description of a quantum system in such a way that they simultaneously provide a basis for a Lie algebra. In other words, the use of symmetry generators for the description of an interacting quantum system incorporates symmetry as a guiding principle to find out the possible solutions. As we demonstrate below, it is always possible to find a language whose generators form a basis of a Lie algebra.

We start by considering the bosonic languages of quantum mechanics. To introduce the definition of a bosonic language, we need to define first the concept of monoid. A monoid is a triple $(\mathfrak{M}, \square, \mathbf{1})$ in which $\mathfrak{M}$ is a non-empty set, $\square$ is an associative product in $\mathfrak{M}$, and $\mathbf{1}$ is an element of $\mathfrak{M}$ such that $\square(\mathbf{1}, \mathfrak{a})=\mathfrak{a}=\square(\mathfrak{a}, \mathbf{1})$ for all $\mathfrak{a} \in \mathfrak{M}$. In this way we see that the the concept of monoid generalizes the notion of group; a group is a monoid all of whose elements are invertible [18]. A bosonic language is a set of operators which can be grouped in subsets $S_{\mathbf{i}}$ (associated to each mode) and satisfy the following conditions:

- Each element $\mathfrak{b}_{\mathbf{i}}^{\mu}$ of $S_{\mathbf{i}}\left(\mu \in\left[1, N_{\mathfrak{g}}\right]\right)$ belongs to the algebra of endomorphisms for the vector space $\mathcal{H}_{\mathbf{i}}$ over the field of complex numbers $\mathbb{C}, \mathfrak{b}_{\mathbf{i}}^{\mu}: \mathcal{H}_{\mathbf{i}} \rightarrow \mathcal{H}_{\mathbf{i}}$, and these elements are linearly independent.

- The elements of $S_{\mathrm{i}}$ generate a monoid of linear transformations under the associative product in the algebra which acts irreducibly on $\mathcal{H}_{\mathbf{i}}$ in the sense that the only subspaces stabilized by $S_{\mathbf{i}}$ are $\mathcal{H}_{\mathbf{i}}$ and $\mathbf{0}$ (0 is the null vector). 
- If $\mathfrak{b}_{\mathfrak{i}}^{\mu}$ and $\mathfrak{b}_{\mathbf{j}}^{\nu}$ are elements of different subsets $S_{\mathbf{i}}$ and $S_{\mathbf{j}}$, then $\mathfrak{b}_{\mathfrak{i}}^{\mu} \mathfrak{b}_{\mathbf{j}}^{\nu}=\square\left(\mathfrak{b}_{\mathfrak{i}}^{\mu}, \mathfrak{b}_{\mathbf{j}}^{\nu}\right)=\square\left(\mathfrak{b}_{\mathbf{j}}^{\nu}, \mathfrak{b}_{\mathfrak{i}}^{\mu}\right)$.

The elements of the sets $S_{\mathrm{i}}$ are called generators of the bosonic language. Combining the associative product and the additive operation in the algebra of endomorphisms of $\mathcal{H}_{\mathbf{i}}$, $\operatorname{End}_{\mathbb{C}} \mathcal{H}_{\mathbf{i}}$, we can define the non-associative Lie product [, ], which is called commutator: $\left[\mathfrak{b}_{\mathbf{i}}^{\mu}, \mathfrak{b}_{\mathbf{j}}^{\nu}\right]=\mathfrak{b}_{\mathbf{i}}^{\mu} \mathfrak{b}_{\mathbf{j}}^{\nu}-\mathfrak{b}_{\mathbf{j}}^{\nu} \mathfrak{b}_{\mathbf{i}}^{\mu}[19]$. Then, the last condition can be reformulated by establishing that the commutator of elements in different subsets is zero

$$
\left[\mathfrak{b}_{\mathbf{i}}^{\mu}, \mathfrak{b}_{\mathbf{j}}^{\nu}\right]=0, \quad \text { if } \mathbf{i} \neq \mathbf{j} .
$$

It is also important to notice that the set $S_{\mathrm{i}}$ is not necessarily closed under the regular product (composition) or the Lie product (commutator). If the set $S_{\mathbf{i}}$ is closed under the Lie product, the elements of $S_{\mathrm{i}}$ generate a Lie algebra. We will denote the Lie algebra by $\mathcal{S}_{\mathrm{i}}$. In addition, since each generator is represented by an endomorphism of $\mathcal{H}_{\mathrm{i}}$ there is a particular representation $\Gamma_{\mathcal{S}}$ of $\mathcal{S}_{\mathrm{i}}$ associated to the bosonic language. The second condition for a bosonic language implies that $\Gamma_{\mathcal{S}}$ is irreducible. The third condition implies that the global Lie algebra associated to the complete set of generators is the direct sum of local algebras $\mathcal{S}_{\mathbf{i}}, \mathcal{S}=\bigoplus_{\mathbf{i}} \mathcal{S}_{\mathbf{i}}$. Therefore, if the set $S_{\mathrm{i}}$ is closed under the Lie product, we can represent the bosonic language by the conjunction of the Lie algebra $\mathcal{S}$, and the irreducible representation $\Gamma_{\mathcal{S}}: \mathcal{S} \wedge \Gamma_{\mathcal{S}}$. The dimension of $\Gamma_{\mathcal{S}}$ is equal to the dimension of the local Hilbert space $\mathcal{H}_{\mathrm{i}}$ : $\operatorname{dim} \Gamma_{\mathcal{S}}=D$. The algebra of endomorphisms for the vector space $\mathcal{H}_{\mathbf{i}}$ is the enveloping algebra of $\mathcal{S}_{\mathbf{i}}$.

The following fundamental theorem shows that two languages are equivalent if they have in common the dimension $D$ of their local Hilbert space $\mathcal{H}_{\mathbf{i}}$, and $D$ is finite. In other words, there is always a dictionary connecting both languages, and a given physical phenomena can be described with either one. A corollary of the theorem is that given a particular language one can always find an equivalent one which can be expressed as $\mathcal{S} \wedge \Gamma_{\mathcal{S}}$, i.e., the operators are generators of a Lie algebra $\mathcal{S}_{\mathrm{i}}$ in the irreducible representation $\Gamma_{\mathcal{S}}$. In general there are various complex Lie algebras which can be used to build equivalent languages. The only condition is that they have to admit an irreducible representation of dimension $D$. Clearly, the number of Lie algebras satisfying this condition increases with $D$.

\section{A. Equivalent Classes of Bosonic Languages}

The demonstration of the fundamental theorem of this section is a direct consequence of the classical theorem of Burnside [20], which plays an important role in the theory of rings. Given the above definitions we can enunciate the theorem in the following way:

Burnside's theorem. Let $G$ be a monoid of linear transformations in a finite dimensional vector space $V$ over an algebraically closed field $F$, that acts irreducibly on $V$ in the sense that the only subspaces stabilized by $G$ are $V$ and $\mathbf{0}$. Then $G$ contains a basis for $\operatorname{End}_{F} V$ over $F$.
$\operatorname{End}_{F} V$ is an abbreviated notation for the ring of endomorphisms of $V$ over the field $F$. In quantum mechanical systems, the field $F$ corresponds to the complex numbers $\mathbb{C}$. The demonstration of the Burnside's theorem can be found, for instance, in Ref. [20]. An immediate consequence of this theorem is the following one which is the basis for connecting different bosonic languages:

Fundamental theorem ("On the equivalence of languages"). Given two bosonic languages having the same finite dimension $D$ of their local Hilbert spaces, $\mathcal{H}_{\mathrm{i}}$, the generators of one of them can be written as a polynomial function of the generators of the other language and vice versa.

Proof: The proof is a trivial application of Burnside's theorem. We need to notice first that since the dimension $D$ is the same for both languages, the spaces $\mathcal{H}_{\mathbf{i}}$ are also the same (vector space of dimension $D$ over the field of complex numbers $\mathbb{C}$ ). Let us consider the monoid of transformations $G_{1}$ generated by multiplying the generators of the first language until the enlarged set becomes closed under the product. The second condition in the definition of a bosonic language states that $G_{1}$ acts irreducibly on $\mathcal{H}_{\mathbf{i}}$. Since the dimension $D$ of $\mathcal{H}_{\mathbf{i}}$ is finite, Burnside's theorem guarantees that $G_{1}$ admits a basis for $\operatorname{End}_{\mathbb{C}} \mathcal{H}_{\mathbf{i}}$. Therefore, any endomorphism in $\mathcal{H}_{\mathbf{i}}$ can be written as a linear combination of endomorphisms in $G_{1}$. In particular, the generators of the second language can be written in this way because they belong to End $\mathcal{H}_{\mathfrak{T}}$. Since each element of $G_{1}$ is a product of generators of the first language, this concludes the demonstration.

This theorem establishes an isomorphism between the algebras of endomorphisms associated to each of the two languages. Motivated by this observation we can introduce the notion of classes of equivalent bosonic languages. We will say that two bosonic languages belong to the same class if they have the same dimension $D$ of their local Hilbert spaces $\mathcal{H}_{\mathrm{i}}$. The fundamental theorem establishes the existence of dictionaries connecting languages within the same class. As a consequence, we can use any bosonic language in the class to describe a given physical phenomena. The natural question which emerges from this result is: What is the most appropriate language in a given class for describing our particular problem? There is no generic answer to this question. Nonetheless, the following two corollaries give an important hint for problems which are invariant under particular transformations, because they relate the notion of language to the generators of symmetry groups.

Corollary I: In each class of bosonic languages there is at least one which is the conjunction of a Lie algebra $\mathcal{S}$ and an irreducible representation $\Gamma_{\mathcal{S}}\left(\mathcal{S} \wedge \Gamma_{\mathcal{S}}\right)$, i.e., the generators of the bosonic language are generators of the Lie algebra $\mathcal{S}_{\mathrm{i}}$ in the representation $\Gamma_{\mathcal{S}}$.

Proof: First, we need to notice that each class is characterized by the dimension $D$ of the local Hilbert space $\mathcal{H}_{\mathbf{i}}$. Let us consider the group $U(1) \otimes S U(2)$. The Lie algebra associated to this group is $\mathcal{L}_{\mathbf{i}}=u(1) \bigoplus s u(2)$. The generators of $\mathcal{L}_{\mathbf{i}}$ are $\left\{\mathbf{1}_{\mathbf{i}}, S_{\mathbf{i}}^{x}, S_{\mathbf{i}}^{y}, S_{\mathbf{i}}^{z}\right\}$,

$$
\left[S_{\mathbf{i}}^{\mu}, S_{\mathbf{j}}^{\nu}\right]=i \delta_{\mathbf{i j}} \epsilon_{\mu \nu \lambda} S_{\mathbf{i}}^{\lambda}, \quad \mu, \nu, \lambda=x, y, z
$$

$\left(S_{\mathbf{i}}^{ \pm}=S_{\mathbf{i}}^{x} \pm i S_{\mathbf{i}}^{y}\right.$ and $\epsilon$ is the totally antisymmetric Levi- 
Civita symbol), and there is one irreducible representation $\Gamma_{\mathcal{L}}^{D}$ of dimension $D$ for each possible value of $D$ [21]. Therefore, the set $\mathcal{S}_{\mathbf{i}}=\left\{\mathbf{1}_{\mathbf{i}}, S_{\mathbf{i}}^{x}, S_{\mathbf{i}}^{y}, S_{\mathbf{i}}^{z}\right\}$ in the representation $\Gamma_{\mathcal{L}}^{D}$ fulfills the three requirements for a bosonic language and operates in a local Hilbert space of dimension $D$. Since $\mathcal{S}_{\mathbf{i}}$ is the set of generators of the Lie algebra $\mathcal{L}_{\mathbf{i}}$, making $\mathcal{S}_{\mathbf{i}}=\mathcal{L}_{\mathbf{i}}$ and $\Gamma_{\mathcal{S}}=$ $\Gamma_{\mathcal{L}}^{D}$, we have proved the corollary I.

The proof of corollary I shows that any bosonic problem with a local Hilbert space of dimension $D$ can be described with $S U(2)$-spins of magnitude (representation) $S=(D-$ 1)/2. The Matsubara-Matsuda (MM) [4] transformation is the simplest application of this corollary to $D=2$. (Indeed, one can construct generalized MM transformations for any $D$ [22].)

We introduce now another definition which is motivated by the next corollary. A given bosonic language will be called hierarchical if any local physical operator $\hat{\mathcal{O}}_{\mathbf{i}}$ can be written as a linear combination of the generators of the language, i.e.,

$$
\hat{\mathcal{O}}_{\mathbf{i}}=\sum_{\mu=1}^{N_{\mathfrak{g}}} \lambda_{\mu} \mathfrak{b}_{\mathbf{i}}^{\mu}
$$

where $\lambda_{\mu} \in \mathbb{C}$, and it is the conjunction of a Lie algebra $\mathcal{S}$ and an irreducible representation $\Gamma_{\mathcal{S}}$.

Corollary II: In each class of bosonic languages there is one which is hierarchical and its generators are the identity and the generators of $s u(N=D)$ in the fundamental representation.

Proof: For each class, with dimension $D$ of the local Hilbert space $\mathcal{H}_{\mathbf{i}}$, we consider the group $U(1) \otimes S U(N)$ with $N=D$. The generators of the Lie algebra associated to this group, $\mathcal{L}_{\mathbf{i}}=u(1) \bigoplus s u(N)$, are the identity plus the generators of $s u(N)$. Since the fundamental representation $\Gamma_{\mathcal{L}}^{F}$ of $\mathcal{L}_{\mathbf{i}}$ has dimension $D=N$, the conjunction of $\mathcal{L}=\bigoplus_{\mathbf{i}} \mathcal{L}_{\mathbf{i}}$ and this representation is one possible language for the class considered. Since the dimension of $\mathcal{L}_{\mathbf{i}}$ is $D^{2}$, which is the dimension of the vector space $\operatorname{End}_{\mathbb{C}} \mathcal{H}_{\mathbf{i}}$, then the generators of $\mathcal{L}_{\mathbf{i}}$ also form a basis for $\operatorname{End}_{\mathbb{C}} \mathcal{H}_{\mathbf{i}}$.

The first consequence of corollary II is that the generators of any language can be expressed as a linear combination of generators of a hierarchical language in the same class. Again, the most trivial example is given by the class of bosonic languages containing the spin $S=1 / 2$ lattice. The generators of any language (like hard-core bosons or any two level system) in the same class can be written as a linear combination of the identity and the Pauli matrices. We will see later that corollary II is the key to get a hierarchical classification of the possible broken symmetries of a given physical Hamiltonian.

We consider now two additional examples that illustrate in detail the contents of the fundamental theorem and the subsequent corollaries. The first example corresponds to hardcore bosons with $N_{f}$ different flavors $\alpha$. Since they are hard core only single occupancy is allowed, i.e., the eigenvalues of $\bar{n}_{\mathbf{i}}=\sum_{\alpha} \bar{n}_{\mathbf{i} \alpha}$ are either 0 or $1\left(\bar{b}_{\mathbf{i} \alpha}^{\dagger} \bar{b}_{\mathbf{i} \beta}^{\dagger}=0\right.$, and $\bar{n}_{\mathbf{i} \alpha}=\bar{b}_{\mathbf{i} \alpha}^{\dagger} \bar{b}_{\mathbf{i} \alpha}$ is the number operator for the flavor $\alpha$ at the site i). To distinguish between site and flavor indices we will adopt the following convention: Sites are denoted by latin indices while the different flavors (or orbitals within the same site) are labeled by greek indices. The minimal set $S_{\mathbf{i}}$ of operators that we can use to generate a bosonic language which is appropriate for hard-core bosons is: $S_{\mathbf{i}}=\left\{\mathbf{1}_{\mathbf{i}}, \bar{b}_{\mathbf{i} \alpha}^{\dagger}, \bar{b}_{\mathbf{i} \alpha}\right\}$ with $1 \leq \alpha \leq N_{f}$. It can be shown that this set satisfies the three requirements for a bosonic language. The dimension of the local Hilbert space for these endomorphisms is $D=N_{f}+1$. Then by varying the total number of flavors we can generate all possible values of $D$. Since each class of bosonic languages is characterized by the value of $D$, these hard-core bosons provide an example of a bosonic language in each class. It is clear that the set $S_{\mathbf{i}}$ is not closed under the Lie product. Therefore, we cannot associate a Lie algebra to this minimal bosonic language. However, if we increase the number of generators in the set $S_{\mathbf{i}}$ by including bilinear forms of the type $\bar{b}_{\mathbf{i} \alpha}^{\dagger} \bar{b}_{\mathbf{i} \beta}$, then the new set $\mathcal{S}_{\mathbf{i}}=\left\{\mathbf{1}_{\mathbf{i}}, \bar{b}_{\mathbf{i} \alpha}^{\dagger}, \bar{b}_{\mathbf{i} \alpha}, \bar{b}_{\mathbf{i} \alpha}^{\dagger} \bar{b}_{\mathbf{i} \beta}\right\}$, with $1 \leq \alpha, \beta \leq N_{f}$, becomes closed under the Lie product

$$
\left\{\begin{array}{l}
{\left[\bar{b}_{\mathbf{i} \alpha}, \bar{b}_{\mathbf{j} \beta}\right]=\left[\bar{b}_{\mathbf{i} \alpha}^{\dagger}, \bar{b}_{\mathbf{j} \beta}^{\dagger}\right]=0,} \\
{\left[\bar{b}_{\mathbf{i} \beta}, \bar{b}_{\mathbf{j} \alpha}^{\dagger}\right]=\delta_{\mathbf{i j}}\left(\delta_{\alpha \beta}-\bar{n}_{\mathbf{i}} \delta_{\alpha \beta}-\bar{b}_{\mathbf{i} \alpha}^{\dagger} \bar{b}_{\mathbf{i} \beta}\right),} \\
{\left[\bar{b}_{\mathbf{i} \alpha}^{\dagger} \bar{b}_{\mathbf{i} \beta}, \bar{b}_{\mathbf{j} \gamma}^{\dagger}\right]=\delta_{\mathbf{i j}} \delta_{\beta \gamma} \bar{b}_{\mathbf{i} \alpha}^{\dagger} .}
\end{array}\right.
$$

This means that the extended set $\mathcal{S}_{\mathbf{i}}$ is now a set of generators for a Lie algebra in a particular representation. From the commutation relations (Eq. (6) we can conclude that $\mathcal{S}_{\mathbf{i}}$ is the direct sum of an $u(1)$ algebra, generated by the identity $\mathbf{1}_{\mathbf{i}}$, and an $\operatorname{su}(N)\left(N=D=N_{f}+1\right)$ algebra generated by $\left\{\bar{b}_{\mathbf{i} \alpha}^{\dagger}, \bar{b}_{\mathbf{i} \alpha}, \bar{b}_{\mathbf{i} \alpha}^{\dagger} \bar{b}_{\mathbf{i} \beta}\right\}: \mathcal{S}_{\mathbf{i}}=u(1) \bigoplus s u(N)$. The representation $\Gamma_{\mathcal{S}}$ is the fundamental representation of $s u(N)$ $\left(\operatorname{dim} \Gamma_{\mathcal{S}}=N\right)$. Therefore, the new language is a hierarchical one. In section $\mathrm{VA}$ we give a more detailed description of this particular language. Here we only want to emphasize the practical consequences of the fundamental theorem and its corollaries. The first non-trivial observation is that for each system of interacting multiflavored hard-core bosons, there is an equivalent system of interacting $S U(N)$-spins in the fundamental representation (with the minimal non-zero magnitude). For $N_{f}=1$ we recover the well-known MM transformation [4]. We can see now that this is the generalization to $s u(N)$ of the MM transformation. With this example we can envision the broad set of applications derived from the fundamental theorem. Another consequence of the theorem is that any physical theory for a bosonic system can be formulated in terms of multiflavored hard-core bosons if the dimension of the local Hilbert space is finite. The usefulness of this formulation will depend on the particular system as it is illustrated in the next sections. Since the second language is hierarchical we can write down any local physical operator (endomorphism in $\mathcal{H}_{\mathbf{i}}$ ) as a linear combination of its generators. Therefore, each of these generators will appear in the Hamiltonian under consideration with a power not larger than one. In addition, if the Hamiltonian has a global symmetry generated by a direct sum of local transformations: $\bigoplus_{\mathbf{i}} \hat{T}_{\mathbf{i}}$, the symmetry will become explicit by writing the Hamiltonian in terms of a hierarchical language. The most basic example is the case of MM hard-core bosons $\left(N_{f}=1\right)$ in a lattice, described by a Hamiltonian with a kinetic energy term and a nearest-neighbor density-density interaction. The expression 
for the Hamiltonian in terms of the first language defined by the set $S_{\mathbf{i}}$ is

$$
H_{\mathrm{xxz}}=t \sum_{\langle\mathbf{i}, \mathbf{j}\rangle}\left(\bar{b}_{\mathbf{i}}^{\dagger} \bar{b}_{\mathbf{j}}+\bar{b}_{\mathbf{j}}^{\dagger} \bar{b}_{\mathbf{i}}\right)+V \sum_{\langle\mathbf{i}, \mathbf{j}\rangle}\left(\bar{n}_{\mathbf{i}}-\frac{1}{2}\right)\left(\bar{n}_{\mathbf{j}}-\frac{1}{2}\right) .
$$

where $\langle\mathbf{i}, \mathbf{j}\rangle$, refers to nearest-neighbors in an otherwise regular $d$-dimensional lattice. Since $\bar{b}_{\mathbf{i}}^{\dagger}$ and $\bar{b}_{\mathbf{i}}$ are not generators of a Lie algebra, the eventual global symmetries of $H_{x x z}$ remain hidden in this particular language. However, if we translate $H_{\mathrm{x} \times \mathrm{z}}$ to the second $S U(2)$-spin language using the dictionary provided by Matsubara and Matsuda [4]

$$
\begin{aligned}
S_{\mathbf{i}}^{z} & =\bar{n}_{\mathbf{i}}-\frac{1}{2}, \\
S_{\mathbf{i}}^{+} & =\bar{b}_{\mathbf{i}}^{\dagger}, \\
S_{\mathbf{i}}^{-} & =\bar{b}_{\mathbf{i}},
\end{aligned}
$$

we can immediately unveil the hidden symmetries of $H_{\mathrm{xxz}}$. The well-known expression for $H_{\mathrm{xxz}}$ in terms of the $s u(2)$ generators (i.e., the equivalent spin Hamiltonian) is

$$
H_{\mathrm{xxz}}=\sum_{\langle\mathbf{i}, \mathbf{j}\rangle} J_{z} S_{\mathbf{i}}^{z} S_{\mathbf{j}}^{z}+\frac{J_{\perp}}{2}\left(S_{\mathbf{i}}^{+} S_{\mathbf{j}}^{-}+S_{\mathbf{i}}^{-} S_{\mathbf{j}}^{+}\right) .
$$

The magnetic couplings, $J_{z}$ and $J_{\perp}$, are related to the original parameters, $t$ and $V$, by the relations: $J_{z}=V$ and $J_{\perp}=2 t$. It is clear from the last version of $H_{x \times z}$ (Eq. 9) that the original model has a global $S U(2)$ invariance if $V=2 t$, i.e., it is in the isotropic Heisenberg point. The existence of this $S U(2)$-symmetric point has a very important consequence for the phase diagram of the bosonic model of Eq. (7): If there is a charge density wave (CDW) instability at that point, the $S U(2)$ invariance implies that there is also a Bose-Einstein condensation and vice versa. The order parameters of both phases are different components of a unique order parameter in the spin language, i.e., the staggered magnetization of the antiferromagnetic phase $(t>0)$. The $z$-component of the staggered magnetization is mapped onto the CDW order parameter for the bosonic gas, while the transverse component is equivalent to the order parameter for the Bose-Einstein condensation. Only one of these two phases, which are coexisting at the $S U(2)$ invariant point, is stable when we depart from the symmetric point in any of both directions in parameter space (Bose-Einstein condensation if $V<2 t$ and CDW if $V>2 t$ ). In this very simple example we can see the advantages of using a hierarchical language (su(2) in this case). In the first place, we can immediately recognize the high symmetry points. Secondly, we can describe an eventual broken symmetry state at those points in terms of a unified order parameter [23]. If we were to use a non-hierarchical language to describe the same problem, we would find coexistence of more than one phase at the high symmetry points. The order parameters of each of those phases are different components of the unified order parameter that we simply found with the hierarchical language. These ideas are developed in more detail in section VIII Here the aim is to give a flavor of the potential applications of our fundamental theorem.
Using the concept of transmutation of statistics, to be introduced in the next section, we will extend the notion of classes of bosonic languages to more general classes containing non-bosonic languages (i.e., languages for which Eq. (3) is replaced by a different algebraic condition). The most wellknown examples of non-bosonic languages are the fermionic ones for which the commutator of Eq. 3. is replaced by an anticommutator. We will see that there are non-local transformations which map non-bosonic languages into bosonic ones. In this way, the simultaneous application of these transformations and the fundamental theorem provides the natural path toward a unification of the different languages used to describe quantum systems.

\section{TRANSMUTATION OF STATISTICS}

In the previous section we have shown that two different bosonic languages can be connected (i.e., there is a dictionary connecting the two languages) if they have the same dimension of their local Hilbert spaces $D$. However, we know that the bosonic languages do not exhaust all the possible languages of quantum mechanics. The best known example is the fermionic language, whose creation and annihilation operators obey a closed set of anticommutation relations. There are many other examples of non-bosonic languages that we address below.

Is it possible to connect these non-bosonic languages to the bosonic ones introduced in the previous section? A positive answer to this question is given in this section by introducing operators which transform commutators into anticommutators and vice versa. These operators have a local (related to the generalized Pauli exclusion principle (see section $\nabla D$ ) and a non-local (related to the exchange statistics) components. The local component is derived at the beginning of this section, while the non-local one is introduced in the second part. We will see that an Abelian anyonic statistics can also be achieved by introducing a continuous parameter in these transformations. (Non-Abelian statistics is beyond the scope of the present manuscript.) The transmutation of statistics together with the fundamental theorem establish the framework needed to classify the classes of equivalent languages. This means that to describe a given physical problem one can use the original language or any other belonging to the same class.

Before proceeding, we would like to mention that it seems as if there are two unrelated notions of anyonic statistics in the literature. One tied to quantum mechanics in first quantization in the coordinate representation, and another derived within the framework of quantum field theory. In both cases the original motivation to introduce such particles was basically as an inherent possibility in the kinematics of $(2+1)$ dimensional quantum mechanics and clearly the concepts, if correctly implemented, should be equivalent but are not indeed [25]. In this manuscript, our anyon notion is consistent with the one developed in quantum field theory where the exclusion properties are preserved under statistical transmutation. In this way, fermions can be kinematically transformed into hard-core bosons but not into canonical ones. 


\section{A. Fermionic Languages}

In addition to bosons, the other type of fundamental particles found in Nature are the fermions. We have seen that the notion of bosonic languages is closely related to the concept of Lie algebras, since for each class of bosonic languages there is at least one language whose elements are generators of a Lie algebra. However, the same cannot be done for fermions. This can be easily seen by noticing that the third condition for a bosonic language (see Eq. (3)) is not valid in general for fermions. In addition, the main consequence of the third condition for a bosonic language is that the global Lie algebra is the direct sum of the local ones associated to each subset $S_{\mathbf{i}}$. The generalization of these concepts to fermionic languages can be done by introducing the notion of Lie superalgebras (see for instance Ref. [26]). The fermionic languages are associated to Lie superalgebras in the same way the bosonic languages are associated to Lie algebras. Therefore, to give a general definition of a fermionic language we should first introduce and explain the notions of Grassman algebras, which are associative superalgebras, and Lie superalgebras. Since this is beyond the scope of the present paper, we will only consider the fermionic language generated by the canonical creation and annihilation operators

$$
\left\{\begin{array}{l}
\left\{c_{\mathbf{i} \alpha}, c_{\mathbf{j} \beta}\right\}=\left\{c_{\mathbf{i} \alpha}^{\dagger}, c_{\mathbf{j} \beta}^{\dagger}\right\}=0 \\
\left\{c_{\mathbf{i} \alpha}, c_{\mathbf{j} \beta}^{\dagger}\right\}=\delta_{\mathbf{i j}} \delta_{\alpha \beta}
\end{array}\right.
$$

and other languages obtained by imposing particular local constraints on the canonical fermions. These generators, together with the identity, generate the Heisenberg Lie superalgebra. In analogy to the bosonic languages (see Eq. (3), the Lie product (in the superalgebra) of two elements acting in different sites (different subsets $\mathcal{S}_{\mathbf{i}}, \mathcal{S}_{\mathbf{j}}$ ) is zero. Thus, instead of having a direct sum of local Lie algebras like in the bosonic case, we have a direct sum of local Lie superalgebras. In the case of canonical fermions the local Lie superalgebras are Heisenberg superalgebras.

\section{B. Local Transmutation}

We will start by considering multiflavor fermions $c_{\mathbf{i} \alpha}^{\dagger}(\alpha \in$ $\left[1, N_{f}\right]$ ) which satisfy the canonical anticommutation relations (Eq. 10). Other types of fermions, usually considered in physics, can be derived from the canonical ones by imposing particular constraints. For this reason, the transformations derived for canonical fermions can be extended to these other fermionic algebras by incorporating those constraints. This procedure is illustrated with different examples in the next section.

The canonical fermions can be transformed into bosons $\tilde{b}_{\mathbf{i} \alpha}^{\dagger}$ which are hard-core in each flavor (the eigenvalues of $\tilde{n}_{\mathbf{i} \alpha}=\tilde{b}_{\mathbf{i} \alpha}^{\dagger} \tilde{b}_{\mathbf{i} \alpha}$ are either 0 or 1 ), i.e., two or more bosons of the same flavor are not allowed on the same site, but two of different flavors are permitted. In the previous section we have shown that a physical theory for objects obeying commutation relations (Lie brackets) can be formulated in terms of a bosonic language. By the present connection we will be able to extend this statement to fermions (or anyons, in general) through a transmutation of statistics. To this end, we need to define a different type of multiflavored hard-core boson $\tilde{b}_{\mathbf{i} \alpha}^{\dagger}$ satisfying the following commutation relations

$$
\left\{\begin{array}{l}
{\left[\tilde{b}_{\mathbf{i} \alpha}, \tilde{b}_{\mathbf{j} \beta}\right]=\left[\tilde{b}_{\mathbf{i} \alpha}^{\dagger}, \tilde{b}_{\mathbf{j} \beta}^{\dagger}\right]=0,} \\
{\left[\tilde{b}_{\mathbf{i} \alpha}, \tilde{b}_{\mathbf{j} \beta}^{\dagger}\right]=\delta_{\mathbf{i j}} \delta_{\alpha \beta}\left(1-2 \tilde{n}_{\mathbf{i} \alpha}\right),\left[\tilde{n}_{\mathbf{i} \alpha}, \tilde{b}_{\mathbf{j} \beta}^{\dagger}\right]=\delta_{\mathbf{i j}} \delta_{\alpha \beta} \tilde{b}_{\mathbf{i} \alpha}^{\dagger},}
\end{array}\right.
$$

which implies $\left\{\tilde{b}_{\mathbf{i} \alpha}, \tilde{b}_{\mathbf{i} \alpha}^{\dagger}\right\}=1$. The Lie algebra generated by these bosonic operators is $\mathcal{L}=\bigoplus_{\alpha, \mathbf{i}} s u(2)$, i.e. each set $\left\{\tilde{b}_{\mathbf{i} \alpha}^{\dagger}, \tilde{b}_{\mathbf{i} \alpha}, \tilde{n}_{\mathbf{i} \alpha}-1 / 2\right\}$ generates an $s u(2)$ algebra.

To show that these bosons (hard-core in each flavor) can be expressed as a function of canonical fermions, one defines

$$
c_{\mathbf{j} \alpha}^{\dagger}=\tilde{b}_{\mathbf{j} \alpha}^{\dagger} \hat{\mathcal{T}}_{\mathbf{j} \alpha}^{\dagger},
$$

where $\hat{\mathcal{T}}_{\mathbf{j} \alpha}=\exp \left[i \pi \sum_{\beta<\alpha} \tilde{n}_{\mathbf{j} \beta}\right]$ is the local transmutator, and we are assuming a particular ordering for the flavor index $\alpha$. From the expression for $\hat{\mathcal{T}}_{\mathbf{j} \alpha}$ it is clear that

$$
\hat{\mathcal{T}}_{\mathbf{j} \alpha}^{2}=I, \quad \hat{\mathcal{T}}_{\mathbf{j} \alpha}^{\dagger}=\hat{\mathcal{T}}_{\mathbf{j} \alpha}
$$

It is straightforward to verify that the $c_{\mathrm{j}}$-operators satisfy local canonical anticommutation relations (Eq. 10 when $\mathbf{i}=\mathbf{j}$ ).

In this way we have established a mapping between fermions and bosons which are operating locally (on a given orbital or mode $\mathbf{j}$ ). In other words, we have related the subset $\mathcal{S}_{\mathbf{j}}=\left\{\tilde{b}_{\mathbf{j} \alpha}^{\dagger}, \tilde{b}_{\mathbf{j} \alpha}, \tilde{n}_{\mathbf{j} \alpha}-1 / 2\right\}$ of local generators of a bosonic language to the subset $\hat{\mathcal{S}}_{\mathbf{j}}=\left\{c_{\mathbf{j} \alpha}^{\dagger}, c_{\mathbf{j} \alpha}, \hat{n}_{\mathbf{j} \alpha}-1 / 2\right\}$ of local generators of canonical fermions $\left(\hat{n}_{\mathbf{j} \alpha}=c_{\mathbf{j} \alpha}^{\dagger} c_{\mathbf{j} \alpha}\right)$.

\section{Non-Local Transmutation}

So far, we have only transmuted the commutation relations between generators which belong to the same site or subset $\mathcal{S}_{\mathbf{i}}$. For commutation relations of two generators of different sites we need to introduce a non-local operator $K_{\mathbf{j}}$. Jordan and Wigner [3] were the first to introduce such an operator in connection to their one-dimensional $(1 d$ or $d=1)$ transformation between spins $S=1 / 2$ and spinless fermions. The so-called kink operator that they introduced is

$$
K_{\mathbf{j}}^{1 d}=\exp \left[i \pi \sum_{\mathbf{l}<\mathbf{j}} \bar{n}_{\mathbf{l}}\right],
$$

where $\bar{n}_{1}$ is the number operator for spinless fermions at the site 1. It is clear that for multiflavor canonical fermions we only need to replace $\bar{n}_{1}$ by $\hat{n}_{1}=\sum_{\alpha} \hat{n}_{1 \alpha}$, where $\alpha$ denotes the flavor. Therefore, Eq. 12 must be replaced by

$$
c_{\mathbf{j} \alpha}^{\dagger}=\tilde{b}_{\mathbf{j} \alpha}^{\dagger} \hat{\mathcal{T}}_{\mathbf{j} \alpha}^{\dagger} K_{\mathbf{j}}^{\dagger}=\tilde{b}_{\mathbf{j} \alpha}^{\dagger} \mathcal{K}_{\mathbf{j} \alpha}^{\dagger},
$$

where $K_{\mathbf{j}}=K_{\mathbf{j}}^{1 d}$ for a $1 d$ lattice. Even though this is a nonlocal operator, it does not introduce long-range interactions if 
the model has only short-range terms [27]. In other words, a given $1 d$ Hamiltonian can be written in terms of bosons, fermions or anyons, and the interactions remain short range for all the cases. This is a special characteristic of one dimension. The only consequence of changing the statistics of the particles is a change of the short-range interactions in the original basis. Therefore, the concept of particle statistics in one dimension becomes irrelevant since any physical system can be described with a bosonic language without changing the short-range character of the interactions [28].

The next step is the generalization of $K_{\mathbf{i}}$ to higher dimensions. This has been done by Fradkin [29] and Y.R. Wang [30], who considered the generalization of the traditional JW transformation for the two-dimensional $(2 d)$ case, and HuertaZanelli [31] and S. Wang [32], who did the same for higher dimensions. We will see that, in the same way we did for the $1 d$ case, these generalizations can be extended to transform canonical fermions into bosons and vice versa. The generalization given by Fradkin [29] for the $2 d$ case is

$$
K_{\mathbf{j}}^{2 d}=\exp \left[i \sum_{\mathbf{l}} a(\mathbf{l}, \mathbf{j}) \bar{n}_{\mathbf{l}}\right]
$$

Here, $a(\mathbf{l}, \mathbf{j})$ is the angle between the spatial vector $\mathbf{l}-\mathbf{j}$ and a fixed direction on the lattice, and $a(\mathbf{j}, \mathbf{j})$ is defined to be zero (see Fig. 11. Again for the case of multiflavor canonical fermions we just need to replace $\bar{n}_{1}$ by $\hat{n}_{1}$. We comment that the $1 d$ kink (or string) operators constitute a particular case of Eq. 16 with $a(\mathbf{l}, \mathbf{j})=\pi$ when $\mathbf{l}<\mathbf{j}$ and equals zero otherwise.

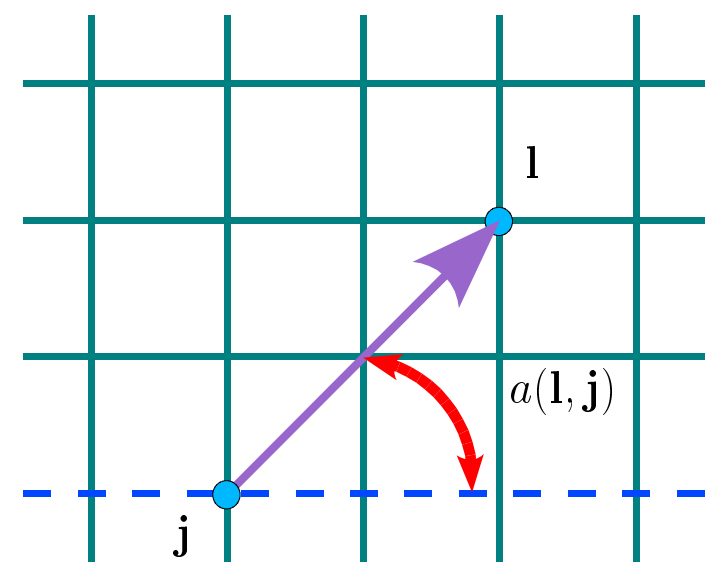

FIG. 1: Schematics of the geometry and function describing one of the possible ways of defining the statistical transmutator $K_{\mathbf{j}}^{2 d}$ in $2 d$. The dotted line represents a fixed direction on the lattice.

A general procedure for finding the string operators in $d$ dimension has been given by Wang [32] in the way we describe below. Following the $1 d$ and $2 d$ cases, the expression proposed for the operator $K_{\mathrm{j}}$ is

$$
K_{\mathbf{j}}=\exp \left[i \sum_{\mathbf{l}} \omega(\mathbf{l}, \mathbf{j}) \bar{n}_{\mathbf{l}}\right]
$$

where $\omega(\mathbf{l}, \mathbf{j})$ is a function to be determined by imposing the transmutation of statistics. It can be shown that this is equivalent to the antisymmetric condition

$$
\begin{aligned}
\exp [i \omega(\mathbf{l}, \mathbf{j})] & =-\exp [i \omega(\mathbf{j}, \mathbf{l})], \text { if } \mathbf{l} \neq \mathbf{j} \\
\omega(\mathbf{l}, \mathbf{l}) & =0
\end{aligned}
$$

The simplest solution for a $1 d$ lattice is

$$
\omega(\mathbf{l}, \mathbf{j})=\pi \Theta(\mathbf{j}-\mathbf{l})
$$

where $\Theta(x)$ is the $1 d$ step or Heaviside function. This is the solution found by Jordan and Wigner (see Eq. (14). The solution introduced in Ref. [29] for the $2 d$ case corresponds to

$$
\omega(\mathbf{l}, \mathbf{j})=a(\mathbf{l}, \mathbf{j})
$$

However, it has been pointed out by Wang [32] that this is not the only possible solution since

$$
\omega(\mathbf{l}, \mathbf{j})=\pi\left[\Theta\left(j_{1}-l_{1}\right)\left(1-\delta_{l_{1} j_{1}}\right)+\Theta\left(j_{2}-l_{2}\right) \delta_{l_{1} j_{1}}\right],
$$

with $\mathbf{l}=l_{1} \mathbf{e}_{1}+l_{2} \mathbf{e}_{2}$ and $\mathbf{j}=j_{1} \mathbf{e}_{1}+j_{2} \mathbf{e}_{2}$, also satisfies Eqs. 18. The advantage of this solution is that its generalization to higher dimensions is straightforward $\left(1=\sum_{\mu} l_{\mu} \mathbf{e}_{\mu}\right.$, and $\mu \in[1, d]$ ). For instance, in $3 d$ we have

$$
\begin{aligned}
\omega(\mathbf{l}, \mathbf{j}) & =\pi\left[\Theta\left(j_{1}-l_{1}\right)\left(1-\delta_{l_{1} j_{1}}\right)\right. \\
& +\Theta\left(j_{2}-l_{2}\right) \delta_{l_{1} j_{1}}\left(1-\delta_{l_{2} j_{2}}\right) \\
& \left.+\Theta\left(j_{3}-l_{3}\right) \delta_{l_{1} j_{1}} \delta_{l_{2} j_{2}}\right] .
\end{aligned}
$$

In the next section we will see that, for $d>1$, the operator $K_{\mathbf{j}}$ introduces non-local interactions in the new representation of the original Hamiltonian. A phase factor of the form $\exp \left[i \int_{\mathbf{x}}^{\mathbf{y}} \mathbf{A} \cdot d \mathbf{s}\right]$ appears in between the product $c_{\mathbf{x}}^{\dagger} c_{\mathbf{y}}$ of the kinetic energy-like terms. The field operator $\mathbf{A}(\mathbf{x})$ is defined by

$$
\mathbf{A}(\mathbf{x})=\sum_{\mathbf{l}} \nabla \omega(\mathbf{x}, \mathbf{l}) \bar{n}_{\mathbf{l}}
$$

where $\nabla$ represents the lattice gradient $(\mu, \nu \in[1, d])$

$$
\nabla_{\mu} \omega(\mathbf{x}, \mathbf{l})=\omega\left(\mathbf{x}+\mathbf{e}_{\mu}, \mathbf{l}\right)-\omega(\mathbf{x}, \mathbf{l}) .
$$

It was shown [29] that $\mathbf{A}(\mathbf{x})$ is the vector potential of a generalized Chern-Simons construction for a lattice. The field strength $F_{\mu \nu}$ associated to this vector potential is the lattice rotor

$$
\begin{aligned}
& F_{\mu \nu}(\mathbf{x})=\nabla_{\mu} A_{\nu}(\mathbf{x})-\nabla_{\nu} A_{\mu}(\mathbf{x}) \\
& F_{0 \mu}(\mathbf{x})=\nabla_{0} A_{\mu}(\mathbf{x})-\nabla_{\mu} A_{0}(\mathbf{x})
\end{aligned}
$$

where $\nabla_{0}$ is the time derivative and $A_{0}(\mathbf{x})$ is a scalar field. Since $A_{0}$ is a Lagrange multiplier field for the Chern-Simons Lagrangian, it can be integrated out to get the Gauss law

$$
\bar{n}_{\mathbf{l}}=\frac{1}{\pi} F_{12}(\mathbf{l}) .
$$


This relation imposes the constraint between charge and flux giving rise to the vector potential $\mathbf{A}$. The physical interpretation is that there is a flux attached to each particle. In this way the phase associated to the original particle statistics is now generated by a mechanism based on the Aharonov-Bohm effect. It can be seen that the $\omega$ function introduced by Fradkin [29] (Eq. (20) generates a vector potential A which is solution of Eq. 26. On the other hand, the $\omega$ function proposed by Wang [32] cannot be associated to any flux since the total change of $\omega$ on any closed loop vanishes (or equivalently $\oint \mathbf{A} . d \mathbf{s}=0)$. This result suggests that the vector potential can be eliminated by a gauge transformation. However, it is impossible to find a gauge transformation which does not change the commutation relations of the particles. The advantage of the solution given by Wang [32] is the straightforward generalization to higher spatial dimensions. Fradkin's approach (2d) [29] provides an alternative formulation of the original problem and has stimulated original methods to find relevant solutions in quantum Hall systems. The generalization of his approach to $3 d$ [31] is more involved since it requires an extended Hilbert space and non-Abelian gauge transformations.

Summarizing, we have shown that one can write down the generators of the canonical fermionic algebra as an operator function of hard-core bosons and then as a function of the generators of any Lie group having an irreducible representation of dimension given by the dimension $D$ of the local Hilbert space. If we are dealing with canonical fermions, the dimension $D$ is equal to $2^{N_{f}}$. However, we can obtain other values of $D$ by imposing different constraints on the fermionic occupation numbers like in the bosonic case [22] (see next Section). This is a common situation for strongly correlated problems where part of the Hilbert space is eliminated when deriving effective low-energy theories. For instance, the Hilbert space of the effective low-energy theory for the Hubbard model ( $t-J$ model) has the constraint of no double-occupancy and therefore $D$ is equal to 3 .

\section{Anyons}

Similarly, one can extend this idea of transmutation of statistics to particles satisfying general equal-time anyonic canonical commutation relations defined by an angle $\theta$. To this end we need to generalize the transmutators to any statistical angle $0 \leq \theta \leq \pi$

$$
\begin{aligned}
\hat{\mathcal{T}}_{\mathbf{j} \alpha}^{\theta} & =\exp \left[i \theta \sum_{\beta<\alpha} n_{\mathbf{j} \beta}\right], \\
K_{\mathbf{j}}^{\theta} & =\exp \left[i \frac{\theta}{\pi} \sum_{\mathbf{l}} \omega(\mathbf{l}, \mathbf{j}) n_{\mathbf{l}}\right]
\end{aligned}
$$

We have seen that there are many types of bosonic particles. For each type we will get a different type of anyon after statistical transmutation. For instance, if we start from canonical bosons $\left(n_{\mathbf{i} \alpha}=b_{\mathbf{i} \alpha}^{\dagger} b_{\mathbf{i} \alpha}\right)$

$$
\left\{\begin{array}{l}
{\left[b_{\mathbf{i} \alpha}, b_{\mathbf{j} \beta}\right]=\left[b_{\mathbf{i} \alpha}^{\dagger}, b_{\mathbf{j} \beta}^{\dagger}\right]=0,} \\
{\left[b_{\mathbf{i} \alpha}, b_{\mathbf{j} \beta}^{\dagger}\right]=\delta_{\mathbf{i j}} \delta_{\alpha \beta},\left[n_{\mathbf{i} \alpha}, b_{\mathbf{j} \beta}^{\dagger}\right]=\delta_{\mathbf{i j}} \delta_{\alpha \beta} b_{\mathbf{i} \alpha}^{\dagger},}
\end{array}\right.
$$

by transmutation of the statistics

$$
a_{\mathbf{j} \alpha}^{\dagger}=b_{\mathbf{j} \alpha}^{\dagger}\left(\hat{\mathcal{T}}_{\mathbf{j} \alpha}^{\theta}\right)^{\dagger}\left(K_{\mathbf{j}}^{\theta}\right)^{\dagger}=b_{\mathbf{j} \alpha}^{\dagger}\left(\mathcal{K}_{\mathbf{j} \alpha}^{\theta}\right)^{\dagger},
$$

one gets anyons obeying commutation relations

$$
\left\{\begin{array}{l}
{\left[a_{\mathbf{j} \alpha}, a_{\mathbf{j} \alpha}\right]=\left[a_{\mathbf{j} \alpha}^{\dagger}, a_{\mathbf{j} \alpha}^{\dagger}\right]=0,} \\
{\left[a_{\mathbf{j} \alpha}, a_{\mathbf{j} \alpha}^{\dagger}\right]=1,}
\end{array}\right.
$$

and deformed commutation relations if both anyonic operators correspond to different sites or flavors. To write down those commutation relations we need first to define a particular ordering for the combined site and flavor indices [33]. In this way, for $(\mathbf{j}, \beta)>(\mathbf{i}, \alpha)$

$$
\left\{\begin{array}{l}
{\left[a_{\mathbf{i} \alpha}, a_{\mathbf{j} \beta}\right]_{\theta}=\left[a_{\mathbf{i} \alpha}^{\dagger}, a_{\mathbf{j} \beta}^{\dagger}\right]_{\theta}=0,} \\
{\left[a_{\mathbf{j} \beta}^{\dagger}, a_{\mathbf{i} \alpha}\right]_{\theta}=0 .}
\end{array}\right.
$$

The requirement of an ordering for the indices comes from the fact that $[A, B]_{\theta}=-\exp [i \theta][B, A]_{-\theta}$. So unless $\exp [i \theta]$ is a real number (i.e., bosons $(\theta=0)$ or fermions $(\theta=\pi)$ ), we need to define a particular index ordering. It is in this ordering that the intrinsic many-body character of the these particles is encoded. In this simple example we can see that the Pauli exclusion principle and the phase associated to the exchange of two particles with different indices (flavor or site), are two distinct and independent concepts. The exclusion behavior is determined by the local commutation relations between operators associated to the same site and flavor. Since these commutation relations are not changed by the transmutation, Eq. 29], the exclusion properties are preserved when we change the statistics of the particles. In our example we can create any number of anyons in the same orbital and flavor because the local commutation relations are the same as the ones for canonical bosons. However, the deformed commutation relations of Eq. 31 indicate that the result of exchanging two anyons with different indices is the multiplication by a phase factor $\exp [i \theta]$. It is this second aspect, not related to the exclusion properties, that decides whether the particles are bosons, fermions, or anyons.

Now we will consider another example of transmutation from bosons to anyons. In this case, we will take the multiflavored hard-core bosons defined by the commutation relations Eq. 11]. The particles obtained after the transmutation of the hard-core bosons will be called type I hard-core anyons

$$
\tilde{a}_{\mathbf{j} \alpha}^{\dagger}=\tilde{b}_{\mathbf{j} \alpha}^{\dagger}\left(\hat{\mathcal{T}}_{\mathbf{j} \alpha}^{\theta}\right)^{\dagger}\left(K_{\mathbf{j}}^{\theta}\right)^{\dagger}=\tilde{b}_{\mathbf{j} \alpha}^{\dagger}\left(\mathcal{K}_{\mathbf{j} \alpha}^{\theta}\right)^{\dagger}
$$

with

$$
\begin{aligned}
\hat{\mathcal{T}}_{\mathbf{j} \alpha}^{\theta} & =\exp \left[i \theta \sum_{\beta<\alpha} \tilde{n}_{\mathbf{j} \beta}\right], \\
K_{\mathbf{j}}^{\theta} & =\exp \left[i \frac{\theta}{\pi} \sum_{\mathbf{l}} \omega(\mathbf{l}, \mathbf{j}) \tilde{n}_{\mathbf{l}}\right] .
\end{aligned}
$$

Like in the previous example, the local commutation relations are preserved $\left(\tilde{n}_{\mathbf{j} \alpha}=\tilde{a}_{\mathbf{j} \alpha}^{\dagger} \tilde{a}_{\mathbf{j} \alpha}, \tilde{n}_{\mathbf{j}}=\sum_{\alpha=1}^{N_{f}} \tilde{n}_{\mathbf{j} \alpha}\right)$

$$
\left\{\begin{array}{l}
{\left[\tilde{a}_{\mathbf{j} \alpha}, \tilde{a}_{\mathbf{j} \alpha}\right]=\left[\tilde{a}_{\mathbf{j} \alpha}^{\dagger}, \tilde{a}_{\mathbf{j} \alpha}^{\dagger}\right]=0,} \\
{\left[\tilde{a}_{\mathbf{j} \alpha}, \tilde{a}_{\mathbf{j} \alpha}^{\dagger}\right]=1-2 \tilde{n}_{\mathbf{j} \alpha} .}
\end{array}\right.
$$


In this particular case, since there is a hard-core condition $\tilde{a}_{\mathbf{j} \alpha}^{\dagger} \tilde{a}_{\mathbf{j} \alpha}^{\dagger}=0$, the operators also satisfy the following local anticommutation relations

$$
\left\{\begin{array}{l}
\left\{\tilde{a}_{\mathbf{j} \alpha}, \tilde{a}_{\mathbf{j} \alpha}\right\}=\left\{\tilde{a}_{\mathbf{j} \alpha}^{\dagger}, \tilde{a}_{\mathbf{j} \alpha}^{\dagger}\right\}=0, \\
\left\{\tilde{a}_{\mathbf{j} \alpha}, \tilde{a}_{\mathbf{j} \alpha}^{\dagger}\right\}=1 .
\end{array}\right.
$$

Thus, the local anticommutation relations are also preserved under statistical transmutation. Clearly, Eqs. 35 are the local anticommutation relations for canonical fermions. This is not surprising since the multiflavored hard-core bosons defined by Eq. 111 can be transmuted into canonical fermions (see Eq. 15). For the commutation relations involving operators with different indices, we have to define an index ordering like in the previous example. For $(\mathbf{j}, \beta)>(\mathbf{i}, \alpha)$

$$
\left\{\begin{array}{l}
{\left[\tilde{a}_{\mathbf{i} \alpha}, \tilde{a}_{\mathbf{j} \beta}\right]_{\theta}=\left[\tilde{a}_{\mathbf{i} \alpha}^{\dagger}, \tilde{a}_{\mathbf{j} \beta}^{\dagger}\right]_{\theta}=0,} \\
{\left[\tilde{a}_{\mathbf{j} \beta}^{\dagger}, \tilde{a}_{\mathbf{i} \alpha}\right]_{\theta}=0 .}
\end{array}\right.
$$

$\theta=0$ corresponds to bosons which are hard-core in each flavor (see Eq. 11), and $\theta=\pi$ to canonical fermions, i.e., $\tilde{a}_{\mathbf{j} \alpha}^{\dagger}(\theta=0)=\tilde{b}_{\mathbf{j} \alpha}^{\dagger}$ and $\tilde{a}_{\mathbf{j} \alpha}^{\dagger}(\theta=\pi)=c_{\mathbf{j} \alpha}^{\dagger}$. For any statistical angle $\theta$ one can put up to a single particle per mode and flavor (i.e, the particles are hard-core in each flavor and, therefore, satisfy the Pauli exclusion principle). It is evident in general that the transmutation of the statistics does not change the local commutation relations and therefore the exclusion rule. In a later section we will show how to generalize the exclusion principle to accommodate up to $p$ particles per single quantum state. In particular, we will see that the paraparticles, which obey this generalized exclusion principle, can be obtained from particles which are partial transmutations of canonical bosons (to obtain the parabosons) or fermions (to obtain the parafermions).

The last example of anyonic particles corresponds to the transmutation of the hard-core bosons defined by the commutation relations Eq. 6. This concept will be useful for the generalized JW transformations [22] that we introduce in the next section (these particles will be called type II hard-core anyons or simply JW particles)

$$
\bar{a}_{\mathbf{j} \alpha}^{\dagger}=\bar{b}_{\mathbf{j} \alpha}^{\dagger}\left(\hat{\mathcal{T}}_{\mathbf{j} \alpha}^{\theta}\right)^{\dagger}\left(K_{\mathbf{j}}^{\theta}\right)^{\dagger}=\bar{b}_{\mathbf{j} \alpha}^{\dagger}\left(\mathcal{K}_{\mathbf{j} \alpha}^{\theta}\right)^{\dagger},
$$

with

$$
\begin{aligned}
\hat{\mathcal{T}}_{\mathbf{j} \alpha}^{\theta} & =\exp \left[i \theta \sum_{\beta<\alpha} \bar{n}_{\mathbf{j} \beta}\right] \\
K_{\mathbf{j}}^{\theta} & =\exp \left[i \frac{\theta}{\pi} \sum_{\mathbf{l}} \omega(\mathbf{l}, \mathbf{j}) \bar{n}_{\mathbf{l}}\right] .
\end{aligned}
$$

Since the local commutation relations are preserved, we have $\left(\bar{n}_{\mathbf{j} \alpha}=\bar{a}_{\mathbf{j} \alpha}^{\dagger} \bar{a}_{\mathbf{j} \alpha}, \bar{n}_{\mathbf{j}}=\sum_{\alpha=1}^{N_{f}} \bar{n}_{\mathbf{j} \alpha}\right)$

$$
\left\{\begin{array}{l}
{\left[\bar{a}_{\mathbf{j} \alpha}, \bar{a}_{\mathbf{j} \alpha}\right]=\left[\bar{a}_{\mathbf{j} \alpha}^{\dagger}, \bar{a}_{\mathbf{j} \alpha}^{\dagger}\right]=0,} \\
{\left[\bar{a}_{\mathbf{j} \alpha}, \bar{a}_{\mathbf{j} \alpha}^{\dagger}\right]=1-\bar{n}_{\mathbf{j} \alpha}-\bar{n}_{\mathbf{j}} .}
\end{array}\right.
$$

Again, we need to define an index ordering for the deformed commutation relations involving operators with different indices. For $(\mathbf{j}, \beta)>(\mathbf{i}, \alpha)$

$$
\left\{\begin{array}{l}
{\left[\bar{a}_{\mathbf{i} \alpha}, \bar{a}_{\mathbf{j} \beta}\right]_{\theta}=\left[\bar{a}_{\mathbf{i} \alpha}^{\dagger}, \bar{a}_{\mathbf{j} \beta}^{\dagger}\right]_{\theta}=0,} \\
{\left[\bar{a}_{\mathbf{j} \beta}^{\dagger}, \bar{a}_{\mathbf{i} \alpha}\right]_{\theta}=0 .}
\end{array}\right.
$$

Notice that, formally, in all cases we could have considered different angles $\theta$ instead of a single one in the expressions for the local, $\hat{\mathcal{T}}_{\mathbf{j} \alpha}^{\theta}$ (e.g., $\theta_{1}$ ), and non-local, $K_{\mathbf{j}}^{\theta}$ (e.g., $\theta_{2}$ ), transmutators, although we do not see how physically relevant this general situation could be.

It is important to stress at this point when the exchange statistics property of the particles (i.e., the property attached to the non-local part of the transmutator) becomes relevant in the description of a particular physical system. It is clear that whenever the system Hamiltonian does not permute particles on different sites $\mathbf{i}$ and $\mathbf{j}$, then $K_{\mathbf{j}}^{\theta}$ becomes a symmetry and the Hamiltonian is invariant under particle exchange statistics. This is the case in many $1 d$ problems (e.g., the $X X Z$ model of Eq. (7) or the Hubbard model) and in special $2 d$ problems such as the $U(1)$ gauge magnet that we expand on in section IXA

Figure 2 summarizes the fundamental notions of languages and dictionaries connecting them, concepts developed in sections $\amalg$ and

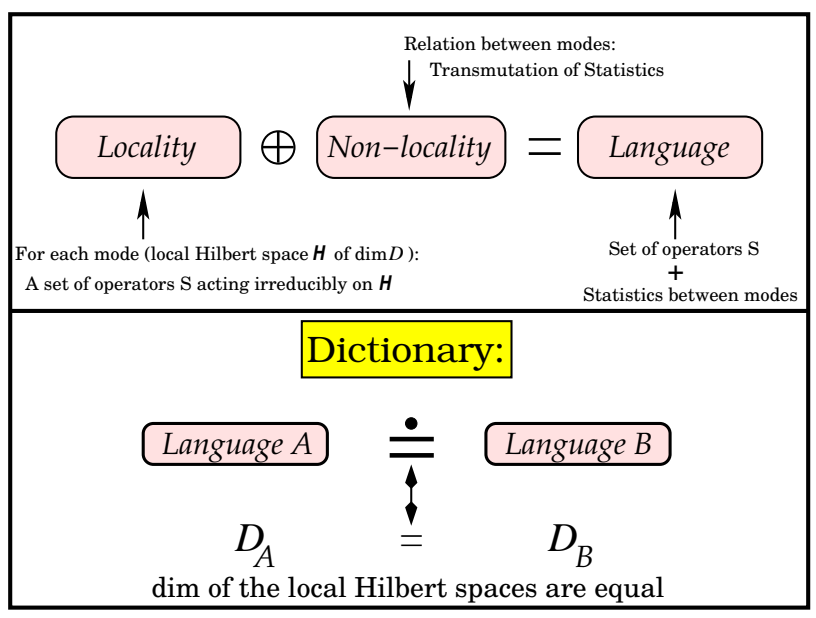

FIG. 2: What is a language, and when can two languages be connected? Summary of the main content of our fundamental theorem in conjunction with the concept of transmutation of statistics.

\section{HILBERT SPACE DECOMPOSITION}

We have seen in the previous section that the only condition to establish an isomorphism between two different languages is that the dimension of their local Hilbert spaces be equal (see Fig. 2). Notice, however, that the choice of local Hilbert space $\mathcal{H}_{\mathbf{i}}$ depends upon the particular decomposition of the global Hilbert space $\mathcal{H}$. When we work on a lattice, the most natural 
decomposition is the one where $\mathcal{H}_{\mathbf{i}}$ is the Hilbert space associated to each site (or mode). This is the decomposition that we have adopted in our previous examples. Another possible decomposition could be the one for which $\mathcal{H}_{\mathbf{i}}$ corresponds to the Hilbert space of a bond (i.e., two sites instead of one). In general, there is no restriction in the way one can partition $\mathcal{H}$.

To be more concrete let us consider a $S=1 / 2$ ladder system (see Fig. 3). The global Hilbert space of the system can be decompose into the direct product of the local Hilbert spaces on each rung. By doing so, the dimension of $\mathcal{H}_{\mathbf{i}}$ is enlarged from $D=2$, for the sites decomposition, to $D=4$. According to our previous results, the change of $D$ opens up the possibility of new mappings between languages. For instance, $D=4$ is the dimension of the on site Hilbert space for canonical $S=1 / 2$ fermions on a lattice. Therefore, the $S=1 / 2$ spin ladder can be mapped onto a system of electrons on a linear chain (see Fig. 3. To be more explicit, a possible isomorphic mapping is (see Eq. 90 for a different mapping)

$$
\begin{aligned}
& c_{\mathbf{j} \uparrow}^{\dagger}=\sqrt{2} S_{\mathbf{j} 2}^{z}\left(S_{\mathbf{j} 1}^{+}+S_{\mathbf{j} 2}^{+}\right) K_{\mathbf{j}}^{\dagger}, \\
& c_{\mathbf{j} \downarrow}^{\dagger}=\sqrt{2}\left(S_{\mathbf{j} 2}^{-}-S_{\mathbf{j} 1}^{-}\right) S_{\mathbf{j} 2}^{z} K_{\mathbf{j}}^{\dagger} .
\end{aligned}
$$

Eq. 41 can be inverted to obtain the expression for the spin operators as a function of the fermionic creation and annihilation operators

$$
\begin{gathered}
\left\{\begin{array}{l}
S_{\mathbf{j} 1}^{+}=\frac{1}{\sqrt{2}}\left[\left(1-2 \hat{n}_{\mathbf{j} \downarrow}\right) c_{\mathbf{j} \uparrow}^{\dagger} K_{\mathbf{j}}+\left(1-2 \hat{n}_{\mathbf{j} \uparrow}\right) K_{\mathbf{j}}^{\dagger} c_{\mathbf{j} \downarrow}\right], \\
S_{\mathbf{j} 1}^{-}=\frac{1}{\sqrt{2}}\left[K_{\mathbf{j}}^{\dagger} c_{\mathbf{j} \uparrow}\left(1-2 \hat{n}_{\mathbf{j} \downarrow}\right)+c_{\mathbf{j} \downarrow}^{\dagger} K_{\mathbf{j}}\left(1-2 \hat{n}_{\mathbf{j} \uparrow}\right)\right], \\
S_{\mathbf{j} 1}^{z}=\frac{1}{2}\left[\hat{n}_{\mathbf{j} \uparrow}-\hat{n}_{\mathbf{j} \downarrow}+c_{\mathbf{j} \downarrow} c_{\mathbf{j} \uparrow}+c_{\mathbf{j} \uparrow}^{\dagger} c_{\mathbf{j} \downarrow}^{\dagger}\right],
\end{array}\right. \\
\left\{\begin{array}{l}
S_{\mathbf{j} 2}^{+}=\frac{1}{\sqrt{2}}\left[c_{\mathbf{j} \uparrow}^{\dagger} K_{\mathbf{j}}+K_{\mathbf{j}}^{\dagger} c_{\mathbf{j} \downarrow}\right], \\
S_{\mathbf{j} 2}^{-}=\frac{1}{\sqrt{2}}\left[K_{\mathbf{j}}^{\dagger} c_{\mathbf{j} \uparrow}+c_{\mathbf{j} \downarrow}^{\dagger} K_{\mathbf{j}}\right], \\
S_{\mathbf{j} 2}^{z}=\frac{1}{2}\left[\hat{n}_{\mathbf{j} \uparrow}-\hat{n}_{\mathbf{j} \downarrow}-c_{\mathbf{j} \downarrow} c_{\mathbf{j} \uparrow}-c_{\mathbf{j} \uparrow}^{\dagger} c_{\mathbf{j} \downarrow}^{\dagger}\right] .
\end{array}\right.
\end{gathered}
$$

In this way we have mapped spin operators acting on a given bond onto fermionic operators acting on the corresponding site (see Fig. 3). This dictionary will be exploited in section VI to map ladder $S=1 / 2$ magnets onto $1 d$ Hubbard-like models.

\section{BRIDGING THE LANGUAGES OF QUANTUM MECHANICS}

The purpose of this section is to illustrate with examples the algebraic framework developed in the previous three sections. In the first part we describe different $S U(N)$ and $S O(N)$ spin-particle mappings which are a direct consequence of our fundamental theorem. In these cases we are connecting bosonic languages and, therefore, it is not necessary to transmute the statistics. In contrast, in the second part we describe the generalization of the JW transformation where the transmutation of the statistics plays a fundamental role. In this case, we connect the different representations of $S U(2)$-spins to constrained fermions [22]. We devote the third part of this section to show how the fractional exclusion statistics algebras emerge from the present formalism in a natural way. We

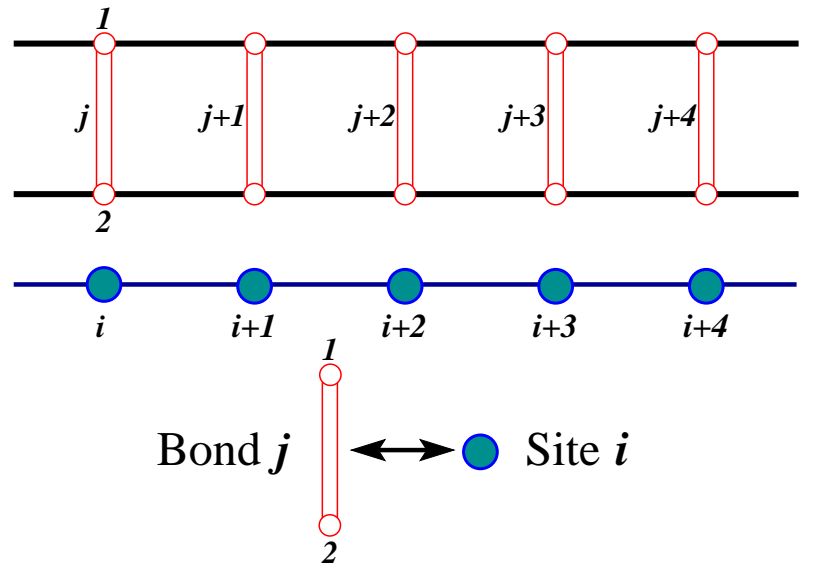

FIG. 3: Mapping between an $S=1 / 2$ ladder and a fermionic linear chain. In this case, bonds (rungs) are mapped onto sites.

close the section showing that the notion of parastatistics introduced by Green [34] is associated to partial transmutations of the canonical bosonic and fermionic languages. In section IX we connect the gauge-field to spin languages through the quantum link model relation. In this way one envisions formal relations between all the different languages used to describe the properties of quantum systems. In the course of this section we will see that many well-known transformations are particular examples of applications of both the fundamental theorem and the transmutation of statistics.

\section{A. $S U(N)$ spin-particle mappings}

\section{Schwinger-Wigner bosons}

The connection between the Schwinger-Wigner bosons and the generators of $s u(N)$ in different irreducible representations results from a simple application of the fundamental theorem. We will consider a system of Schwinger-Wigner bosons with $N_{f}=N$ different flavors $(\alpha \in[1, N]$ ). These bosons obey canonical commutation relations

$$
\left\{\begin{array}{l}
{\left[\hat{b}_{\mathbf{i} \alpha}, \hat{b}_{\mathbf{j} \beta}\right]=\left[\hat{b}_{\mathbf{i} \alpha}^{\dagger}, \hat{b}_{\mathbf{j} \beta}^{\dagger}\right]=0,} \\
{\left[\hat{b}_{\mathbf{i} \alpha}, \hat{b}_{\mathbf{j} \beta}^{\dagger}\right]=\delta_{\mathbf{i j}} \delta_{\alpha \beta}}
\end{array}\right.
$$

and are characterized by the constraint

$$
\sum_{\alpha=1}^{N} \hat{b}_{\mathbf{j} \alpha}^{\dagger} \hat{b}_{\mathbf{j} \alpha}=M
$$

which sets the dimension of the local Hilbert space $\mathcal{H}_{\mathbf{j}}, D=$ $\left(\begin{array}{c}N+M-1 \\ M\end{array}\right)$. For $N=2$ we get $D=M+1$, which means that we can get any value of $D$ by varying $M$. We also know that the Lie algebra $s u(2)$ has one irreducible representation for each value of $D$. In this case $D=2 S+1$, where $S$ is the spin of the representation. According to the fundamental theorem, 
if $2 S+1=M+1$ we can write down the generators of $s u(2)$ $\left(S^{x}, S^{y}, S^{z}\right.$ ) in the $S=M / 2$ representation as a polynomial function of the Schwinger-Wigner bosons and vice versa. The explicit form of these relations is the well-known connection between $S U(2)$-spins and the two-flavor Schwinger-Wigner bosons $(\alpha=1,2)$

$$
\begin{aligned}
S_{\mathbf{j}}^{z} & =\frac{1}{2}\left(\hat{b}_{\mathbf{j} 1}^{\dagger} \hat{b}_{\mathbf{j} 1}-\hat{b}_{\mathbf{j} 2}^{\dagger} \hat{b}_{\mathbf{j} 2}\right), \\
S_{\mathbf{j}}^{+} & =\hat{b}_{\mathbf{j} 1}^{\dagger} \hat{b}_{\mathbf{j} 2}, \\
S_{\mathbf{j}}^{-} & =\hat{b}_{\mathbf{j} 2}^{\dagger} \hat{b}_{\mathbf{j} 1} .
\end{aligned}
$$

According to the fundamental theorem the inverse transformation also exists. The creation and annihilation operators for Schwinger-Wigner bosons $\hat{b}_{\mathbf{i} \alpha}^{\dagger}, \hat{b}_{\mathbf{i} \alpha}$ can be written as a polynomial function of $S_{\mathbf{j}}^{x}, S_{\mathbf{j}}^{y}, S_{\mathbf{j}}^{z}$. However, since the SchwingerWigner bosons are not directly associated to the description of any physical system the inverse transformation is, apparently, not very useful. For this reason, the only application of the Schwinger-Wigner bosons is to provide a different framework for solving spin Hamiltonians. For instance, it is possible to approximately solve the isotropic Heisenberg model by a mean-field approximation which preserves the $S U(2)$ invariance of the model. This symmetry is violated in the usual spin-wave approximation where the spins are described by Holstein-Primakoff bosons. Through this simple example we see yet another important advantage of having different languages to describe the same problem: Different languages provide different frameworks to obtain approximate or exact solutions. Physics provides innumerable examples of applications of this statement. It is not our intention to enumerate all of them in this paper, but to show the operative way to build the dictionaries connecting all of those different languages.

To find the connection between the generators of the $s u(N)$ algebra and the Schwinger-Wigner bosons we just need to consider the general case of arbitrary number of flavors. To simplify the analysis we can start by considering the minimum value of $M$, i.e., $M=1$ (i.e., the fundamental representation). In this case the dimension of the local Hilbert space is $D=\left(\begin{array}{c}N \\ 1\end{array}\right)=N$. Since $N$ is also the dimension of the fundamental representation of $s u(N)$, the fundamental theorem states that the generators of $s u(N)$ can be written as a function of the Schwinger-Wigner bosons. Again, this connection is very well-known and can be explicitly written as

$$
\mathcal{S}^{\alpha \beta}(\mathbf{j})=\hat{b}_{\mathbf{j} \alpha}^{\dagger} \hat{b}_{\mathbf{j} \beta}
$$

Using Eqs. (43) and 44 we can verify that the $\mathcal{S}^{\alpha \beta}$ 's are the generators of the $s u(N)$ algebra in the fundamental representation, i.e., they satisfy the following commutation relations

$$
\left[\mathcal{S}^{\alpha \alpha^{\prime}}(\mathbf{i}), \mathcal{S}^{\beta \beta^{\prime}}(\mathbf{j})\right]=\delta_{\mathbf{i j}}\left[\delta_{\alpha^{\prime} \beta} \mathcal{S}^{\alpha \beta^{\prime}}(\mathbf{j})-\delta_{\alpha \beta^{\prime}} \mathcal{S}^{\beta \alpha^{\prime}}(\mathbf{j})\right],
$$

and operate in an $N$-dimensional vector space. As in the $N=2$ case, by considering larger values of $M$ we obtain higher-order representations of $s u(N)$. The relation between the $s u(N)$ generators and the Schwinger-Wigner bosons is the one given by Eq. (46) independently of the representation.

\section{Fundamental irrep of su(N) and Jordan-Wigner particles}

The fundamental (quark) representation of $s u(N)$ can be mapped onto an algebra of constrained fermions $\left(\bar{c}_{\mathbf{j} \alpha}^{\dagger}=\right.$ $\left.\bar{a}_{\mathbf{j} \alpha}^{\dagger}(\theta=\pi)\right)$ or hard-core bosons $\left(\bar{b}_{\mathbf{j} \alpha}^{\dagger}=\bar{a}_{\mathbf{j} \alpha}^{\dagger}(\theta=0)\right)$ with $N_{f}=N-1$ flavors

$$
\begin{aligned}
\mathcal{S}^{\alpha \beta}(\mathbf{j}) & =\bar{a}_{\mathbf{j} \alpha}^{\dagger} \bar{a}_{\mathbf{j} \beta}-\frac{\delta_{\alpha \beta}}{N} \\
\mathcal{S}^{\alpha 0}(\mathbf{j}) & =\bar{a}_{\mathbf{j} \alpha}^{\dagger} K_{\mathbf{j}}^{\theta}, \mathcal{S}^{0 \beta}(\mathbf{j})=\left(K_{\mathbf{j}}^{\theta}\right)^{\dagger} \bar{a}_{\mathbf{j} \beta} \\
\mathcal{S}^{00}(\mathbf{j}) & =\frac{N_{f}}{N}-\sum_{\alpha=1}^{N_{f}} \bar{n}_{\mathbf{j} \alpha}=-\sum_{\alpha=1}^{N_{f}} \mathcal{S}^{\alpha \alpha}(\mathbf{j}),
\end{aligned}
$$

where $1 \leq \alpha, \beta \leq N_{f}$ runs over the set of particle flavors, and $\bar{a}_{\mathbf{j} \alpha}^{\dagger}=\tilde{a}_{\mathbf{j} \alpha}^{\dagger} \prod_{\beta=1}^{N_{f}}\left(1-\tilde{n}_{\mathbf{j} \beta}\right)$. $\mathcal{S}^{\mu \nu}(\mathbf{j})$ (with $0 \leq \mu, \nu \leq$ $N_{f}$ ) are the components of the $S U(N)$-spin (i.e., there are $N^{2}-1$ linear independent components). It is easy to verify that these are generators of an $s u(n)$ Lie algebra satisfying the commutation relations

$$
\left[\mathcal{S}^{\mu \mu^{\prime}}(\mathbf{j}), \mathcal{S}^{\nu \nu^{\prime}}(\mathbf{j})\right]=\delta_{\mu^{\prime} \nu} \mathcal{S}^{\mu \nu^{\prime}}(\mathbf{j})-\delta_{\mu \nu^{\prime}} \mathcal{S}^{\nu \mu^{\prime}}(\mathbf{j})
$$

For instance, for $N=3$ we have [35] $(\alpha=1,2)$

$$
\mathcal{S}(\mathbf{j})=\left(\begin{array}{ccc}
\frac{2}{3}-\bar{n}_{\mathbf{j}} & \left(K_{\mathbf{j}}^{\theta}\right)^{\dagger} \bar{a}_{\mathbf{j} 1} & \left(K_{\mathbf{j}}^{\theta}\right)^{\dagger} \bar{a}_{\mathbf{j} 2} \\
\bar{a}_{\mathbf{j} 1}^{\dagger} K_{\mathbf{j}}^{\theta} & \bar{n}_{\mathbf{j} 1}-\frac{1}{3} & \bar{a}_{\mathbf{j} 1}^{\dagger} \bar{a}_{\mathbf{j} 2} \\
\bar{a}_{\mathbf{j} 2}^{\dagger} K_{\mathbf{j}}^{\theta} & \bar{a}_{\mathbf{j} 2}^{\dagger} \bar{a}_{\mathbf{j} 1} & \bar{n}_{\mathbf{j} 2}-\frac{1}{3}
\end{array}\right) .
$$

We can immediately see that the $2 \times 2$ block matrix $\mathcal{S}^{\alpha \beta}(\mathbf{j})$ $(1 \leq \alpha, \beta \leq 2)$ contains the generators of $s u(2)$. In general, from the commutation relations Eq. (49), we can verify that if $\mathcal{S}^{\mu \nu}(\mathbf{j})$ are the generators of $s u(N)$, then $\mathcal{S}^{\alpha \beta}(\mathbf{j})$ are the generators of the subalgebra $s u(N-1)$. This will be useful in section VI

\section{Generalization to other irreps of $s u(N)$}

Here we show that the hard-core bosons $\bar{b}_{\mathbf{j} \alpha}$ can be connected to other irreducible representations of $s u(N)$. The dimension of an $s u(N)$ representation of spin $S=M / N$ is $\left(\begin{array}{c}N+M-1 \\ M\end{array}\right)$. The number of flavors is then $N_{f}=\left(\begin{array}{c}N+M-1 \\ M\end{array}\right)-1$. In this case, it is more convenient to adopt the following notation for the flavors: Each flavor $\alpha$ will be denoted by an array of $N$ integer numbers $\left\{m_{1}^{\alpha}, m_{2}^{\alpha}, \cdots, m_{N}^{\alpha}\right\}$ satisfying $\sum_{\sigma=1}^{N} m_{\sigma}^{\alpha}=M$. There are $\left(\begin{array}{c}N+M-1 \\ M\end{array}\right)$ arrays satisfying this condition. However, there is a particular one, $\left\{m_{1}^{0}, m_{2}^{0}, \cdots, m_{N}^{0}\right\}$, associated to the vacuum state.

$$
\begin{aligned}
\mathcal{S}^{\mu \nu}(\mathbf{j}) & =-\delta_{\mu \nu} S+\sum_{\alpha=1}^{N_{f}}\left(g_{\mu \nu}^{\alpha 0} \bar{b}_{\mathbf{j} \alpha}^{\dagger}+g_{\mu \nu}^{0 \alpha} \bar{b}_{\mathbf{j} \alpha}\right) \\
& +\sum_{\alpha, \beta=1}^{N_{f}} g_{\mu \nu}^{\alpha \beta} \bar{b}_{\mathbf{j} \alpha}^{\dagger} \bar{b}_{\mathbf{j} \beta},
\end{aligned}
$$


with

$$
\begin{aligned}
g_{\mu \nu}^{\alpha \beta}= & \delta_{\mu \nu} \delta_{\alpha \beta} m_{\mu}^{\alpha}+\delta_{\left(m_{\mu}^{\alpha}-1\right) m_{\mu}^{\beta}} \delta_{\left(m_{\nu}^{\alpha}+1\right) m_{\nu}^{\beta}} \\
& \times \prod_{\sigma=1, \sigma \neq \alpha, \beta}^{N} \delta_{m_{\mu}^{\sigma} m_{\nu}^{\sigma}}
\end{aligned}
$$

Again, it is easy to verify that these are generators of an $s$ Lie algebra satisfying the commutation relations Eq. 49

This is just an example to show that the generators Lie algebra in a particular and arbitrary representation c mension $D$ can be used to describe a system where $D$ i dimension of the local Hilbert space. For each particular there will be representations which will be more approp (easier to handle) than others. For instance, this set of $s \imath$ representations can be performed in a more natural way $\iota$ Schwinger-Wigner bosons.

\section{B. $S O(N)$ spin-particle mappings}

Another possible language related to the JW particles $\mathrm{i}$ $S O(N)$-spin language. By this name we mean the lang whose generators are the identity and the generators o $s o(N)$ Lie algebra (whose number is $N(N-1) / 2$ ). It is to verify that the following set of operators

$$
\begin{aligned}
\mathcal{M}^{\alpha \beta}(\mathbf{j}) & =\bar{a}_{\mathbf{j} \alpha}^{\dagger} \bar{a}_{\mathbf{j} \beta}-\bar{a}_{\mathbf{j} \beta}^{\dagger} \bar{a}_{\mathbf{j} \alpha}, \\
\mathcal{M}^{0 \beta}(\mathbf{j}) & =\left(K_{\mathbf{j}}^{\theta}\right)^{\dagger} \bar{a}_{\mathbf{j} \beta}-\bar{a}_{\mathbf{j} \beta}^{\dagger} K_{\mathbf{j}}^{\theta}, \\
\mathcal{M}^{00}(\mathbf{j}) & =0
\end{aligned}
$$

obey the $S O(N)$-spin commutation relations

$$
\begin{aligned}
{\left[\mathcal{M}^{\mu \mu^{\prime}}(\mathbf{i}), \mathcal{M}^{\nu \nu^{\prime}}(\mathbf{j})\right] } & =\delta_{\mathbf{i j}}\left[\delta_{\mu^{\prime} \nu} \mathcal{M}^{\mu \nu^{\prime}}(\mathbf{j})-\delta_{\mu^{\prime} \nu^{\prime}} \mathcal{M}^{\mu \nu}(\mathbf{j})\right. \\
& \left.-\delta_{\mu \nu} \mathcal{M}^{\mu^{\prime} \nu^{\prime}}(\mathbf{j})+\delta_{\mu \nu^{\prime}} \mathcal{M}^{\mu^{\prime} \nu}(\mathbf{j})\right],
\end{aligned}
$$

with $0 \leq \mu, \nu \leq N$ and $1 \leq \alpha, \beta \leq N_{f}=N-1$ The antisymmetric relation $\mathcal{M}^{\mu \nu}=-\mathcal{M}^{\nu \mu}$ is immediately derived from the definition of $\mathcal{M}^{\mu \nu}$, Eq. [53). In this way, we have constructed another possible language connected to hard-core particles. Like in the previous case, the subset of operators $\mathcal{M}^{\alpha \beta}$ is a set of generators for an $s o(N-1)$ subalgebra of so $(N)$.

In the following, for simplicity, we will only consider the connection between $S O(N)$-spins and hard-core bosons $\bar{b}_{\mathbf{j} \alpha}=\bar{a}_{\mathbf{j} \alpha}(\theta=0)$ with non-local transmutator $K_{\mathbf{j}}^{\theta}=1$. For instance, for $N=3$, the generators are $(\alpha=1,2)$

$$
\mathcal{M}(\mathbf{j})=\left(\begin{array}{ccc}
0 & \bar{b}_{\mathbf{j} 1}-\bar{b}_{\mathbf{j} 1}^{\dagger} & \bar{b}_{\mathbf{j} 2}-\bar{b}_{\mathbf{j} 2}^{\dagger} \\
\bar{b}_{\mathbf{j} 1}^{\dagger}-\bar{b}_{\mathbf{j} 1} & 0 & \bar{b}_{\mathbf{j} 1}^{\dagger} \bar{b}_{\mathbf{j} 2}-\bar{b}_{\mathbf{j} 2}^{\dagger} \bar{b}_{\mathbf{j} 1} \\
\bar{b}_{\mathbf{j} 2}^{\dagger}-\bar{b}_{\mathbf{j} 2} & \bar{b}_{\mathbf{j} 2}^{\dagger} \bar{b}_{\mathbf{j} 1}-\bar{b}_{\mathbf{j} 1}^{\dagger} \bar{b}_{\mathbf{j} 2} & 0
\end{array}\right)
$$

\section{Generalized Jordan-Wigner Particles}

In 1928 Jordan and Wigner related the spin quantum mechanical degree of freedom to spinless particles with fermion

\section{Classical spins to lattice gas or binary alloy mappings}

Ising Model: (magnetism)
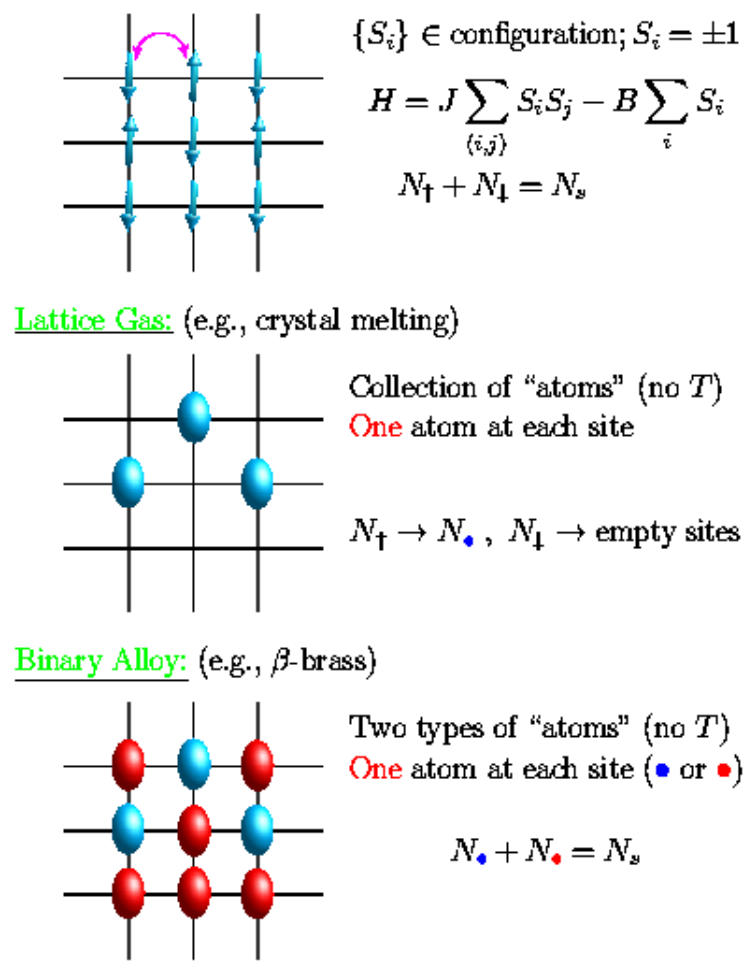

FIG. 4: Classical version of the Jordan-Wigner particles defined in [22]. Here the mapping is performed between the simple lattice of Ising spins $S_{i}= \pm 1$ and a lattice gas of one type of atom or a binary alloy. $N_{\sigma}$, with $\sigma=\uparrow, \downarrow$, is the number of Ising spins of type $\sigma$ while $N_{\bullet}$ is the number of hard-core atoms of flavor $\bullet$ with no kinetic energy $T$.

statistics. This transformation involves the $S=1 / 2$ irreducible representation of the Lie algebra $s u(2)$. We have recently [22] generalized this one-to-one mapping to any irreducible spin representation of $s u(2)$, spatial dimension of the lattice and particle exchange statistics. From the physical viewpoint the JW particles are essentially hard-core independently of their flavor index (i.e., satisfy the Pauli exclusion principle with $p=2$ (see below)).

The generalized JW mappings constitute a kind of quantum version of the well-known classical spin-lattice-gas transformations [36] (see Fig. 4). In a classical lattice gas (or binary alloy) each site can be occupied by at most one atom, a hardcore condition equivalent to its quantum counterpart. On the other hand, since the spin system is classical there is no intrinsic dynamics and the kinetic energy of the gas $(T)$ must be included a posteriori in an ad-hoc fashion. This contrasts the quantum case where the dynamics of the JW particles is intrinsic.

We have seen in section 1 that the canonical fermions can be transformed into bosons which are hard-core in each flavor. We will consider now another type of fermions which 
naturally emerge from the strong coupling limit of models for interacting electrons. If the short range component of the Coulomb repulsion is much larger than the kinetic energy, the repulsion can be effectively replaced by a constraint of no double occupancy. This perturbative approach is usually implemented by a canonical transformation, which leads to an effective Hamiltonian acting on the subspace of states with no double occupancy. The fermionic subalgebra used to describe this effective model is generated by the so-called constrained fermions. Therefore, the constrained fermions are obtained by imposing to the canonical fermions a local constraint of no more than one particle per orbital (or site). This constraint may be incorporated into the fermionic algebra by defining the following creation and annihilation operators for the constrained fields

$$
\bar{c}_{\mathbf{j} \sigma}^{\dagger}=c_{\mathbf{j} \sigma}^{\dagger} \prod_{\tau \in \mathcal{F}_{\eta}}\left(1-\hat{n}_{\mathbf{j} \tau}\right), \bar{c}_{\mathbf{j} \sigma}=\prod_{\tau \in \mathcal{F}_{\eta}}\left(1-\hat{n}_{\mathbf{j} \tau}\right) c_{\mathbf{j} \sigma},
$$

where $\mathcal{F}_{\eta}$ is the set of flavors, with $\eta=\frac{1}{2}, 1$ depending upon the spin character of the irreducible representation. It is easy to check that the particles generated by this fermionic algebra satisfy the constraint of single occupancy, i.e., the eigenvalues of $\bar{n}_{\mathbf{j}}=\sum_{\sigma=1}^{N_{f}} \bar{n}_{\mathbf{j} \sigma}$ are either 0 or 1 . The most well-known context where these fermions appear in condensed matter physics is the strong coupling limit of the Hubbard model, which leads to the $t-J$ Hamiltonian. While the Hubbard model is described in terms of spin- $1 / 2$ canonical fermions (electrons), the fermionic language for the $t-J$ model is generated by creation and annihilation operators of spin- $1 / 2$ constrained fermions $\left(\sigma, \sigma^{\prime}=\uparrow, \downarrow\right)$

$$
\begin{aligned}
& \left\{\bar{c}_{\mathbf{i} \sigma}, \bar{c}_{\mathbf{j} \sigma^{\prime}}\right\}=\left\{\bar{c}_{\mathbf{i} \sigma}^{\dagger}, \bar{c}_{\mathbf{j} \sigma^{\prime}}^{\dagger}\right\}=0, \\
& \left\{\bar{c}_{\mathbf{i} \sigma}, \bar{c}_{\mathbf{j} \sigma^{\prime}}^{\dagger}\right\}=\delta_{\mathbf{i} \mathbf{j}}\left[\bar{c}_{\mathbf{j} \sigma^{\prime}}^{\dagger} \bar{c}_{\mathbf{j} \sigma}+\delta_{\sigma \sigma^{\prime}}\left(1-\bar{n}_{\mathbf{j}}\right)\right] .
\end{aligned}
$$

In this section we will consider the general case of constrained fermions with $N_{f}=2 S$ different flavors. In the context of our previous example, this generalization can be interpreted as the natural language for a $t-J$ model with more than one orbital per site $\mathbf{j}$ or larger-spin fermions. The set of commutation relations for only two different flavors, Eq. 57, is generalized in the following way

$$
\begin{aligned}
& \left\{\bar{c}_{\mathbf{i} \sigma}, \bar{c}_{\mathbf{j} \sigma^{\prime}}\right\}=\left\{\bar{c}_{\mathbf{i} \sigma}^{\dagger}, \bar{c}_{\mathbf{j} \sigma^{\prime}}^{\dagger}\right\}=0, \\
& \left\{\bar{c}_{\mathbf{i} \sigma}, \bar{c}_{\mathbf{j} \sigma^{\prime}}^{\dagger}\right\}=\delta_{\mathbf{i} \mathbf{j}} \begin{cases}1+\bar{n}_{\mathbf{j} \sigma}-\bar{n}_{\mathbf{j}} & \text { if } \sigma=\sigma^{\prime}, \\
\bar{c}_{\mathbf{j} \sigma^{\prime}}^{\dagger} \bar{c}_{\mathbf{j} \sigma} & \text { if } \sigma \neq \sigma^{\prime}\end{cases}
\end{aligned}
$$

Notice that $\prod_{\tau \in \mathcal{F}_{\eta}}^{\tau \neq \sigma}\left(1-\bar{n}_{\mathbf{j} \tau}\right)=1+\bar{n}_{\mathbf{j} \sigma}-\bar{n}_{\mathbf{j}}$ with number operators satisfying $\bar{n}_{\mathbf{j} \sigma} \bar{n}_{\mathbf{j} \sigma^{\prime}}=\delta_{\sigma \sigma^{\prime}} \bar{n}_{\mathbf{j} \sigma}$.

We may now ask what is the bosonic language obtained from the constrained fermions when the statistics is transmuted, i.e.,

$$
\bar{b}_{\mathbf{j} \alpha}^{\dagger}=\bar{c}_{\mathbf{j} \alpha}^{\dagger} K_{\mathbf{j}}^{\pi} .
$$

(We do not need to include the local transmutator since the constraint does not allow to have more than one particle per site (or orbital).) The answer to the question becomes clear if we compare the set of commutation relations for the hard-core bosons, Eq. [6, to the set of anticommutation relations for the constrained fermions, Eq. 58. All anticommutators which are not of the form $\left\{\bar{c}_{\mathbf{i} \sigma}, \bar{c}_{\mathbf{j} \sigma^{\prime}}^{\dagger}\right\}$ are mapped onto the corresponding commutators for hard-core bosons, Eq. (6). This results from the transmutator $K_{\mathbf{j}}^{\pi}$. What is the effect of the transmutation on commutators containing bilinear forms in the creation and annihilation operators? First, it is easy to check that in this case the commutator $\left[\bar{b}_{\mathbf{i} \alpha}^{\dagger} \bar{b}_{\mathbf{i} \beta}, \bar{b}_{\mathbf{j} \gamma}^{\dagger}\right]=\delta_{\mathbf{i j}} \delta_{\beta \gamma} \bar{b}_{\mathbf{i} \alpha}^{\dagger}$ of bosonic operators is mapped onto the corresponding commutator of fermionic operators

$$
\left[\bar{c}_{\mathbf{i} \alpha}^{\dagger} \bar{c}_{\mathbf{i} \beta}, \bar{c}_{\mathbf{j} \gamma}^{\dagger}\right]=\delta_{\mathbf{i j}} \delta_{\beta \gamma} \bar{c}_{\mathbf{i} \alpha}^{\dagger} .
$$

Finally, to complete this correspondence between commutation and anticommutation relations we have to consider the effect of the transmutation on the commutators $\left[\bar{b}_{\mathbf{j} \sigma}, \bar{b}_{\mathbf{j} \sigma}^{\dagger}\right]$ and the anticommutators $\left\{\bar{c}_{\mathbf{j} \sigma}, \bar{c}_{\mathbf{j} \sigma \sigma}^{\dagger}\right\}$. It is easy to check that the transmutation does not change these particular products, i.e.,

$$
\left\{\bar{b}_{\mathbf{i} \beta}, \bar{b}_{\mathbf{i} \alpha}^{\dagger}\right\}=\delta_{\alpha \beta}\left(1-\bar{n}_{\mathbf{i}}\right)+\bar{b}_{\mathbf{i} \alpha}^{\dagger} \bar{b}_{\mathbf{i} \beta} .
$$

We can see that the anticommutator $\left\{\bar{b}_{\mathbf{i} \beta}, \bar{b}_{\mathbf{i} \alpha}^{\dagger}\right\}$ is a linear combination of generators of the Lie algebra defined by the set of commutation relations, Eq. (6). This is so since the set of generators $\left\{I, \bar{b}_{\mathbf{i} \alpha}^{\dagger}, \bar{b}_{\mathbf{i} \alpha}, \bar{b}_{\mathbf{i} \alpha}^{\dagger} \bar{b}_{\mathbf{i} \beta}\right\}$ belongs to the fundamental representation of the Lie algebra.

Therefore, in this case, the generators of the Lie algebra associated to the bosonic language are transmuted into generators of a Lie superalgebra associated to the constrained fermions. The Lie product in the Lie algebra, Eq. (6), is turned into the graded Lie product in the superalgebra. The generators of the bosonic Lie algebra can be separated into two subsets which are mapped onto the odd and the even generators of the fermionic Lie superalgebra. In this particular case, the Lie algebra associated to the commutation relations, Eq. (6), is $u(1) \bigoplus s u(N)$. The creation and annihilation operators for the hard core bosons, $\bar{b}_{\mathbf{i} \alpha}^{\dagger}$ and $\bar{b}_{\mathbf{i} \alpha}$ are mapped onto the odd generators of the Lie superalgebra, $\bar{c}_{\mathbf{i} \alpha}^{\dagger}$ and $\bar{c}_{\mathbf{i} \alpha}$, while the identity and the bilinear forms, $I$ and $\bar{b}_{\mathbf{i} \beta}^{\dagger} \bar{b}_{\mathbf{i} \alpha}$ are mapped onto the even generators $I$ and $\bar{c}_{\mathbf{i} \beta}^{\dagger} \bar{c}_{\mathbf{i} \alpha}$. The bosons which are hard-core in each flavor, $\tilde{b}_{\mathbf{i} \alpha}$, and the canonical fermions, $c_{\mathbf{i} \alpha}$, provide another example of transmutation of a Lie algebra into a Lie superalgebra. In this case, the generators of the Lie algebra $u(1) \bigoplus_{\alpha=1}^{N_{f}} s u(2)$ are transmuted into the generators of a Heisenberg Lie superalgebra.

In this way, through the transmutation of statistics we have established a direct connection between the multiflavored constrained fermions and the multiflavored hard-core bosons. Using this connection we can see that the MM transformation [4] can be obtained from the JW transformation [3] by a simple transmutation of the statistics.

In the course of demonstrating the Corollary I, we have shown that for each class of languages characterized by the dimension $D$ of the local Hilbert space, there is one whose generators are also generators of the Lie algebra $u(1) \bigoplus s u(2)$ 
$(D=2 S+1)$. In addition, we have seen that there is another language in the class whose generators are the identity and the creation and annihilation operators for multiflavored hardcore bosons $\left(N_{f}=D-1\right)$. Then, according to the fundamental theorem, the three components $\left\{S_{\mathbf{i}}^{x}, S_{\mathbf{i}}^{y}, S_{\mathbf{i}}^{z}\right\}$ of a spin $S$ can be written as a polynomial function of $\left\{I_{\mathbf{i}}, \bar{b}_{\mathbf{i} \alpha}^{\dagger}, \bar{b}_{\mathbf{i} \alpha}\right\}$ with $1 \leq \alpha \leq N_{f}$ and vice versa. This is the generalization of the MM [4] transformation to any irreducible spin- $S$ representation of $s u(2)$. Adding now this result to the mapping already established between the multiflavored hard-core bosons and the constrained fermions, we can conclude that it is also possible to write $\left\{S_{\mathbf{i}}^{x}, S_{\mathbf{i}}^{y}, S_{\mathbf{i}}^{z}\right\}$ as a polynomial function of the identity and the creation and annihilation operators of multiflavored constrained fermions and vice versa. The later is the generalization of the JW transformation to any spin $S$ [22]. On the other hand, the generalized JW spin-fermion mapping can be easily extended to include a spin-anyon mapping simply by using the anyonic particles generated by $\bar{a}_{\mathbf{j} \sigma}^{\dagger}$ and $\bar{a}_{\mathbf{j} \sigma}$ (see Eq. [37) [22]. The explicit form of this generalization is given by [22, 37]:

$\underline{\text { Half-odd integer spin } S}\left(\sigma \in \mathcal{F}_{\frac{1}{2}}=\{-S+1, \ldots, S\}\right)$ :

$$
\begin{aligned}
S_{\mathbf{j}}^{+} & =\eta_{\bar{S}} \bar{a}_{\mathbf{j} \bar{S}+1}^{\dagger} K_{\mathbf{j}}^{\theta}+\sum_{\substack{\sigma \in \mathcal{F}_{\frac{1}{2}} \\
\sigma \neq S}} \eta_{\sigma} \bar{a}_{\mathbf{j} \sigma+1}^{\dagger} \bar{a}_{\mathbf{j} \sigma}, \\
S_{\mathbf{j}}^{-} & =\eta_{\bar{S}}\left(K_{\mathbf{j}}^{\theta}\right)^{\dagger} \bar{a}_{\mathbf{j} \bar{S}+1}+\sum_{\substack{\sigma \in \mathcal{F}_{\frac{1}{2}} \\
\sigma \neq S^{-}}} \eta_{\sigma} \bar{a}_{\mathbf{j} \sigma}^{\dagger} \bar{a}_{\mathbf{j} \sigma+1}, \\
S_{\mathbf{j}}^{z}= & -S+\sum_{\sigma \in \mathcal{F}_{\frac{1}{2}}}(S+\sigma) \bar{n}_{\mathbf{j} \sigma}, \\
\bar{a}_{\mathbf{j} \sigma}^{\dagger}= & \left(K_{\mathbf{j}}^{\theta}\right)^{\dagger} L_{\sigma}^{\frac{1}{2}}\left(S_{\mathbf{j}}^{+}\right)^{\sigma+S} \mathcal{P}_{\mathbf{j}}^{\frac{1}{2}}, \\
\text { where } & \mathcal{P}_{\mathbf{j}}^{\frac{1}{2}}=\prod_{\tau \in \mathcal{F}_{\frac{1}{2}}} \frac{\tau-S_{\mathbf{j}}^{z}}{\tau+S}, L_{\sigma}^{\frac{1}{2}}=\prod_{\tau=-S}^{\sigma-1} \eta_{\tau}^{-1} .
\end{aligned}
$$

$\underline{\text { Integer spin } S}\left(\sigma \in \mathcal{F}_{1}=\{-S, \ldots,-1,1, \ldots, S\}\right)$ :

$$
\begin{aligned}
& S_{\mathbf{j}}^{+}=\eta_{0}\left(\bar{a}_{\mathbf{j} 1}^{\dagger} K_{\mathbf{j}}^{\theta}+\left(K_{\mathbf{j}}^{\theta}\right)^{\dagger} \bar{a}_{\mathbf{j} \overline{1}}\right)+\sum_{\substack{\sigma \in \mathcal{F}_{1} \\
\sigma \neq-1, S}} \eta_{\sigma} \bar{a}_{\mathbf{j} \sigma+1}^{\dagger} \bar{a}_{\mathbf{j} \sigma}, \\
& S_{\mathbf{j}}^{-}=\eta_{0}\left(\left(K_{\mathbf{j}}^{\theta}\right)^{\dagger} \bar{a}_{\mathbf{j} 1}+\bar{a}_{\mathbf{j} \overline{1}}^{\dagger} K_{\mathbf{j}}^{\theta}\right)+\sum_{\substack{\sigma \in \mathcal{F}_{1} \\
\sigma \neq-1, S}} \eta_{\sigma} \bar{a}_{\mathbf{j} \sigma}^{\dagger} \bar{a}_{\mathbf{j} \sigma+1}, \\
& S_{\mathbf{j}}^{z}=\sum_{\sigma \in \mathcal{F}_{1}} \sigma \bar{n}_{\mathbf{j} \sigma}, \\
& \bar{a}_{\mathbf{j} \sigma}^{\dagger}=\left(K_{\mathbf{j}}^{\theta}\right)^{\dagger} L_{\sigma}^{1} \begin{cases}\left(S_{\mathbf{j}}^{+}\right)^{\sigma} \mathcal{P}_{\mathbf{j}}^{1} & \text { if } \sigma>0, \\
\left(S_{\mathbf{j}}^{-}\right)^{\sigma} \mathcal{P}_{\mathbf{j}}^{1} & \text { if } \sigma<0\end{cases} \\
& \text { where } \quad \mathcal{P}_{\mathbf{j}}^{1}=\prod_{\tau \in \mathcal{F}_{1}} \frac{\tau-S_{\mathbf{j}}^{z}}{\tau}, L_{\sigma}^{1}=\prod_{\tau=0}^{|\sigma|-1} \eta_{\tau}^{-1},
\end{aligned}
$$

and $\eta_{\sigma}=\sqrt{(S-\sigma)(S+\sigma+1)}$ (see Fig. 5]. [A bar in a subindex means the negative of that number (e.g., $\bar{\sigma}=-\sigma)$.]

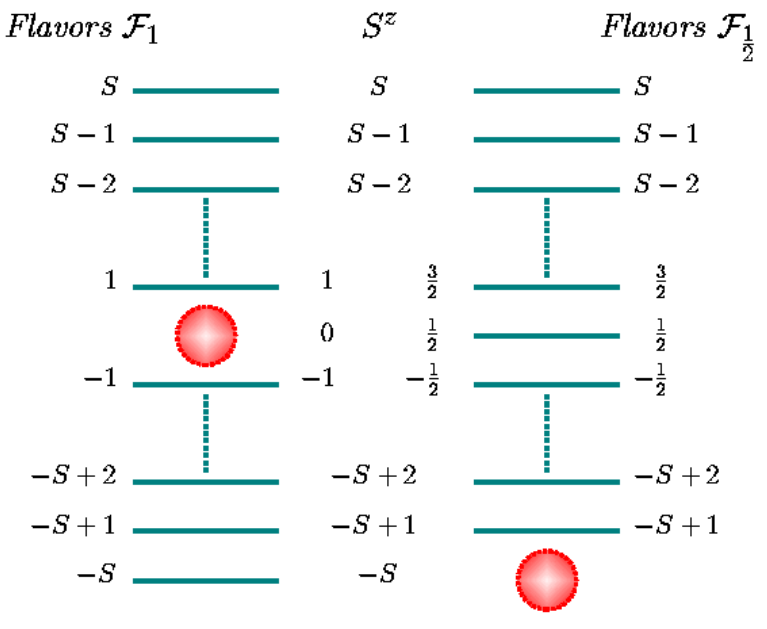

Integer $S \quad$ Half-odd Integer $S$

FIG. 5: Constrained anyon states per site for integer and half-odd integer spin $S$. In both cases there are $2 S$ flavors and the corresponding $2 S+1$ values of $S^{z}$ are shown in the middle column. One degree of freedom is assigned to the anyon vacuum (circle) whose relative position depends upon the spin being integer or half-odd integer.

The total number of flavors is $N_{f}=2 S$, and the $S=1 / 2$ case simply reduces to the traditional JW transformation. These mappings enforce the condition on the Casimir operator $\mathbf{S}_{j}^{2}=$ $S(S+1)$.

\section{Fractional Exclusion Statistics Algebras}

What microscopic properties of the fundamental particle constituents determine the thermodynamic behavior of matter? It has been known since the early days of quantum mechanics that particles in nature are either bosons or fermions in the three-dimensional space. It was immediately realized that for a given interaction among particles the properties of matter were completely different depending upon the constituents being bosons or fermions (for example, normal Coulomb matter is stable thanks to the fermionic nature of its particles). Moreover, it has been known for a long time [38] that there are other theoretical possibilities that interpolate between fermions and bosons, i.e., cases where $p>1$ identical particles occupy one and the same state. Quantum statistics was the name given to this phenomenon clearly nonexistent in the classical physics description of matter.

In the seventies it was realized that two space dimensions allowed for other exotic possibilities with particles having fractional spin and statistics [39]. Later on, this concept proved to be not merely an intellectual exercise since particles obeying such quantum statistics were experimentally realized in the context of the quantum Hall effect (QHE). It was Haldane [40], to our knowledge, the first to realize that the Pauli exclusion principle and exchange statistics ideas were in principle independent concepts. In this way, motivated by 
the properties of quasiparticles in the fractional QHE, he generalized the Pauli exclusion principle introducing the concept of fractional exclusion statistics.

Basically, two physical features characterize the quantum statistics of particles: One is related to their indistinguishability and is characterized by the property that when two identical particles are exchanged the total wave function acquires a phase factor (exchange or permutation statistics). Another refers to the ability to accommodate $p$ particles in the same single-particle quantum state (exclusion statistics) [41]. The first concept depends upon the space dimensionality of the system while the second one is independent and, therefore, unrelated to the notion of anyonic fractional statistics which is applicable exclusively in two space dimensions. For instance, from the generalized Pauli principle viewpoint there is no distinction between particles obeying the algebra of Eqs. (39) and 40. for any statistical angle $\theta$, i.e., all of them correspond to $p=1$. However, the exchange of two particles $\bar{a}$ does depend upon $\theta$.

In this section we consider the problem of formulating the algebra satisfied by these generalized Pauli-exclusion particles and its connection to our fundamental theorem. Our definition is a possible second-quantized version of Haldane's definition introduced at the first quantization level.

To simplify matters we consider as an example a single flavor (single site) fractional exclusion statistics algebra

$$
\left\{\begin{array}{l}
{[g, g]=\left[g^{\dagger}, g^{\dagger}\right]=0} \\
{\left[g, g^{\dagger}\right]=1-F,\left[g^{\dagger} g, g^{\dagger}\right]=g^{\dagger}}
\end{array}\right.
$$

where the operator $F\left(F^{\dagger}=F\right)$ is a polynomial function of $g^{\dagger}$ and $g$, such that $g^{\dagger} F=0$. The $F$ term represents a sort of deformation of the canonical boson algebra (deformed Heisenberg algebras play an important role in the theory of representations of quantum groups). For example, $F$ can be $\frac{p !}{p+1} F=\left(g^{\dagger}\right)^{p}(g)^{p}=\prod_{j=0}^{p-1}\left(g^{\dagger} g-j\right)$ and $p$ is an arbitrary integer with the condition $p>0$. For $g$-particles with this $F$ operator it is very easy to prove that they satisfy the nilpotency condition

$$
\left(g^{\dagger}\right)^{p+1}=0
$$

which means that one can put up to $p$ particles on each mode. $\mathrm{A}(p+1)$-dimensional matrix representation of this algebra is

$$
g=\left(\begin{array}{cccccc}
0 & 1 & 0 & 0 & \cdots & 0 \\
0 & 0 & \sqrt{2} & 0 & \cdots & 0 \\
0 & 0 & 0 & \sqrt{3} & \cdots & 0 \\
\vdots & \vdots & \vdots & \vdots & \vdots & \vdots \\
0 & 0 & 0 & 0 & \cdots & \sqrt{p} \\
0 & 0 & 0 & 0 & \cdots & 0
\end{array}\right)
$$

Certainly, one can build infinitely many different algebras leading to different types of fractional exclusion statistics particles with the same value of $p$. For example, consider the $s u(2)$ algebra generated by the operators $\left\{\bar{g}_{\mathbf{i}}^{\dagger}, \bar{g}_{\mathbf{i}}, n_{\mathbf{i}}^{g}\right\}$ (with $\left.\left(n_{\mathbf{i}}^{g}\right)^{\dagger}=n_{\mathbf{i}}^{g}\right)$ in the $S=1$ (triplet) representation $\left(\left(\bar{g}_{\mathbf{i}}^{\dagger}\right)^{3}=0\right.$, i.e., $p=2$ )

$$
\left\{\begin{array}{l}
{\left[\bar{g}_{\mathbf{i}}, \bar{g}_{\mathbf{j}}\right]=\left[\bar{g}_{\mathbf{i}}^{\dagger}, \bar{g}_{\mathbf{j}}^{\dagger}\right]=0,} \\
{\left[\bar{g}_{\mathbf{i}}, \bar{g}_{\mathbf{j}}^{\dagger}\right]=\delta_{\mathbf{i} \mathbf{j}}\left(1-n_{\mathbf{i}}^{g}\right),\left[n_{\mathbf{i}}^{g}, \bar{g}_{\mathbf{j}}^{\dagger}\right]=\delta_{\mathbf{i j}} \bar{g}_{\mathbf{j}}^{\dagger}}
\end{array}\right.
$$

It is straightforward to realize how these particles relate to the usual spin generators of $s u(2)$

$$
\begin{aligned}
S_{\mathbf{j}}^{+} & =\sqrt{2} \bar{g}_{\mathbf{j}}^{\dagger}, \\
S_{\mathbf{j}}^{-} & =\sqrt{2} \bar{g}_{\mathbf{j}}, \\
S_{\mathbf{j}}^{z} & =n_{\mathbf{j}}^{g}-1,
\end{aligned}
$$

which can be seen as a possible generalization of the MM [4] transformation for $S=1$.

For example, if we consider the $1 d X Y$ model in the $S=1$ spin representation

$$
H_{\mathrm{xy}}=J \sum_{\mathbf{j}}\left(S_{\mathbf{j}}^{x} S_{\mathbf{j}+1}^{x}+S_{\mathbf{j}}^{y} S_{\mathbf{j}+1}^{y}\right),
$$

it is simply related to the $g$-particle Hamiltonian

$$
\begin{aligned}
H_{\mathrm{xy}} & =J \sum_{\mathbf{j}}\left(\bar{g}_{\mathbf{j}}^{\dagger} \bar{g}_{\mathbf{j}+1}+\bar{g}_{\mathbf{j}+1}^{\dagger} \bar{g}_{\mathbf{j}}\right) \\
& =2 J \sum_{\mathbf{k}} \cos \mathbf{k} \bar{g}_{\mathbf{k}}^{\dagger} \bar{g}_{\mathbf{k}}
\end{aligned}
$$

where $\bar{g}_{\mathbf{k}}^{\dagger}=\left(1 / \sqrt{N_{s}}\right) \sum_{\mathbf{j}} \exp (i \mathbf{k} \cdot \mathbf{j}) \bar{g}_{\mathbf{j}}^{\dagger}$ is the Fouriertransformed operator.

\section{E. Green's Parastatistics}

Since the very beginnings of quantum mechanics people tried to understand the depth and consequences of the symmetrization postulate which asserts that physical states of identical particles must be either symmetric (Bose statistics) or antisymmetric (Fermi statistics) under permutations. In 1953 H.S. Green [34] considered the possibility of having non-identical but dynamically similar particles satisfying what is now known as parastatistics. The introduction of parastatistics allowed some hope to represent all particle fields in terms of a fundamental spinor field and, of course, canonical fermions and bosons represented particular cases (parastatistics of order $p=1$ ).

Soon, Greenberg [42] suggested that quarks satisfy parastatistics of order 3 and Green [43] reformulated the neutrino theory of light identifying the neutrinos with parafermions of order 2. It is not our intention to discuss the physical aspects of these assertions but to present parastatistics as another operator language which allows representation of Lie algebras and to relate these particles to the ones already introduced in previous Sections.

Following Green [34, 43], let us introduce the auxiliary modes (upper (lower) sign will be used to define the 
parafermions (parabosons))

$$
\left\{\begin{array}{l}
{\left[d_{\mathbf{i}}^{\alpha}, d_{\mathbf{j}}^{\alpha}\right]_{ \pm}=\left[\check{d}_{\mathbf{i}}^{\alpha}, \check{d}_{\mathbf{j}}^{\alpha}\right]_{ \pm}=0} \\
{\left[d_{\mathbf{i}}^{\alpha}, \check{d}_{\mathbf{j}}^{\alpha}\right]_{ \pm}=\delta_{\mathbf{i j}}}
\end{array}\right.
$$

labeled by the Green indices $\alpha=1, \cdots, p$ and $\mathbf{j}=$ $1, \cdots, N$ and where $\check{d}_{\mathbf{j}}^{\alpha}=\left(d_{\mathbf{j}}^{\alpha}\right)^{\dagger}$, together with the condition $d_{\mathbf{j}}^{\alpha} \mid$ vacuum $\rangle=0, \forall(\mathbf{j}, \alpha)$. For $\alpha \neq \beta$ the auxiliary modes satisfy non-standard relations, i.e.,

$$
\left\{\begin{array}{l}
{\left[d_{\mathbf{i}}^{\alpha}, d_{\mathbf{j}}^{\beta}\right]_{\mp}=\left[\check{d}_{\mathbf{i}}^{\alpha}, \check{d}_{\mathbf{j}}^{\beta}\right]_{\mp}=0} \\
{\left[d_{\mathbf{i}}^{\alpha}, \check{d}_{\mathbf{j}}^{\beta}\right]_{\mp}=0}
\end{array}\right.
$$

Parafermion and paraboson creation and annihilation operators are defined in terms of the auxiliary modes by

$$
d_{\mathbf{j}}^{\dagger}=\sum_{\alpha=1}^{p} \check{d}_{\mathbf{j}}^{\alpha}, d_{\mathbf{j}}=\sum_{\alpha=1}^{p} d_{\mathbf{j}}^{\alpha}
$$

and consequently satisfy the commutation relations

$$
\left\{\begin{array}{l}
{\left[\left[d_{\mathbf{i}}^{\dagger}, d_{\mathbf{j}}\right]_{\mp}, d_{\mathbf{l}}\right]_{-}=-2 \delta_{\mathbf{i l}} d_{\mathbf{j}}} \\
{\left[\left[d_{\mathbf{i}}, d_{\mathbf{j}}\right]_{\mp}, d_{\mathbf{l}}\right]_{-}=0}
\end{array}\right.
$$

It easy to verify that one can define a set of commutative number operators $n_{\mathbf{j}}^{d}\left(n_{\mathbf{j}}^{\alpha}=\check{d}_{\mathbf{j}}^{\alpha} d_{\mathbf{j}}^{\alpha}\right)$ by

$$
n_{\mathbf{j}}^{d}=\frac{1}{2}\left(\left[d_{\mathbf{j}}^{\dagger}, d_{\mathbf{j}}\right]_{\mp} \pm p\right)=\sum_{\alpha=1}^{p} n_{\mathbf{j}}^{\alpha},
$$

which satisfy the commutation rules

$$
\left[n_{\mathbf{i}}^{d}, d_{\mathbf{j}}^{\dagger}\right]_{-}=\delta_{\mathbf{i j}} d_{\mathbf{j}}^{\dagger}
$$

Note that in the parafermionic case $\left(n_{\mathbf{j}}^{\alpha}\right)^{2}=n_{\mathbf{j}}^{\alpha}$, which implies that $n_{\mathbf{j}}^{d}$ has eigenvalues ranging from 0 to $p$. Moreover,

$$
\left(d_{\mathbf{j}}^{\dagger}\right)^{p}=p ! \prod_{\alpha=1}^{p} \check{d}_{\mathbf{j}}^{\alpha}
$$

such that $\left(d_{\mathbf{j}}^{\dagger}\right)^{p+1}=0$, which in a sense also generalizes the Pauli exclusion principle. It is clear from these definitions that canonical fermions (bosons) are parafermions (parabosons) of order $p=1$.

To connect these para-particles to the ones already described in previous sections, one has to realize that (total or partial) local and non-local transmutations are necessary. It is easy to see that the parafermionic auxiliary modes are

$$
\check{d}_{\mathbf{i}}^{\alpha}=c_{\mathbf{i} \alpha}^{\dagger} \exp \left[i \pi\left(\sum_{\mathbf{j}<\mathbf{i}} \sum_{\beta \neq \alpha} \hat{n}_{\mathbf{j} \beta}+\sum_{\beta<\alpha} \hat{n}_{\mathbf{i} \beta}\right)\right],
$$

where a partial non-local transmutation connects $\check{d}_{\mathbf{j}}^{\alpha}$ to the canonical multiflavored fermions $c_{\mathbf{j} \alpha}^{\dagger}$, Eq. 10. Similarly, the parabosonic auxiliary fields are obtained as

$$
\check{d}_{\mathbf{i}}^{\alpha}=b_{\mathbf{i} \alpha}^{\dagger} \exp \left[i \pi\left(\sum_{\mathbf{j}<\mathbf{i}} \sum_{\beta \neq \alpha} n_{\mathbf{j} \beta}+\sum_{\beta<\alpha} n_{\mathbf{i} \beta}\right)\right],
$$

in terms of canonical multiflavored bosons $b_{\mathbf{j} \alpha}^{\dagger}$, Eq. (28).

One can represent all Lie algebras in terms of parafermion or paraboson creation and annihilation operators. Several authors contributed to this observation in the early sixties and seventies [44]. In the following we just present as an example the isomorphism between the algebra of $N$ parafermions and the proper orthogonal complex Lie algebra $B_{N}$ (i.e., the complexification of $s o(2 N+1))$ of dimension $N(2 N+1)$. The set of operators antisymmetric in their indices $(\mathbf{m}, \mathbf{n} \in[1,2 N])$

$$
\left\{\begin{array}{l}
L_{\mathbf{m n}}=-L_{\mathbf{n m}}=i\left(\ell_{\mathbf{n}} \ell_{\mathbf{m}}-\ell_{\mathbf{m}} \ell_{\mathbf{n}}\right),(\mathbf{m}<\mathbf{n}) \\
L_{\mathbf{m} 0}=-L_{0 \mathbf{m}}=\ell_{\mathbf{m}}, \\
L_{00}=0
\end{array}\right.
$$

where

$$
\ell_{2 \mathbf{j}-1}=\frac{d_{\mathbf{j}}^{\dagger}+d_{\mathbf{j}}}{2}, \ell_{2 \mathbf{j}}=\frac{d_{\mathbf{j}}^{\dagger}-d_{\mathbf{j}}}{2 i},
$$

represents a basis which span the Lie algebra isomorphic to $B_{N}\left(\mu, \mu^{\prime}, \nu, \nu^{\prime} \in[0,2 N]\right)$

$$
\begin{aligned}
{\left[L_{\mu \mu^{\prime}}, L_{\nu \nu^{\prime}}\right] } & =i\left(-\delta_{\mu^{\prime} \nu} L_{\mu \nu^{\prime}}+\delta_{\mu^{\prime} \nu^{\prime}} L_{\mu \nu}\right. \\
& \left.+\delta_{\mu \nu} L_{\mu^{\prime} \nu^{\prime}}-\delta_{\mu \nu^{\prime}} L_{\mu^{\prime} \nu}\right)
\end{aligned}
$$

\section{EQUIVALENT CLASSES OF MODELS}

In the previous section we have shown how the different languages of quantum mechanics are connected. How can we exploit these dictionaries to get a better understanding of different physical phenomena? There are many different answers to this question which we will develop in the next sections. In particular, the present section is devoted to show the equivalence between models which, in principle, describe completely different systems [45]. In other words, we show that models describing different physical problems are associated to the same Hamiltonian written in different languages. In this way, these dictionaries appear as an essential tool to connect distinct fields of physics. Preliminary applications of these concepts are given in Refs. [22, 46]. In Ref. [22], we showed explicit connections between spin $S=1$ and $t$ - $J$-like models. In Ref. [46] we demonstrated the equivalence between $S U(3)$ ferromagnetism, coexistence of magnetism and Bose-Einstein condensation in an interacting bosonic gas, and coexistence of a spin-nematic phase and ferromagnetism for a spin one system. The choice of examples in the present section $\nabla 1$ is by no means exhaustive, it is simply illustrative. Table \ summarizes some of the most celebrated mappings known today.

Another interesting aspect of these mappings is the possibility of unveiling hidden symmetries which lead to exact or quasi-exact solutions. By exact solvability we mean that the full spectral problem is reduced to an algebraic procedure, while problems which are quasi-exactly solvable are those which admit only a partial determination of the spectrum by an algebraic procedure (see Fig. 6). Sometimes there are particular languages which allow us to recognize invariant subspaces of our Hamiltonian. The restricted action the 


\begin{tabular}{|c|c|c|c|}
\hline$d$ & Model A & Model B & $M-S$ \\
\hline 1 & Isotropic $X Y(S=1 / 2)$ & $t$ & C - E \\
\hline 1 & Anisotropic $X Y(S=1 / 2)$ & $t-\Delta$ & $C-E$ \\
\hline 1 & $X X Z(S=1 / 2)$ & $t-V$ & $\mathrm{C}-\mathrm{E}(\mathrm{BA})$ \\
\hline 1 & $X X Z(S=1 / 2)$ & $t-J_{z}$ & $\mathrm{P}-\mathrm{E}(\mathrm{BA})$ \\
\hline 1 & $X Y Z(S=1 / 2)$ & $t-\Delta-V$ & $\mathrm{C}-\mathrm{E}(\mathrm{BA})$ \\
\hline 1 & Majumdar-Ghosh $(S=1 / 2)$ & $t-t^{\prime}-V-V^{\prime}$ & C - GS \\
\hline 1 & BB $S=1(\phi=\pi / 4, \mathrm{LS})$ & $t-\bar{J}-V-\mu$ & $\mathrm{C}-\mathrm{E}(\mathrm{BA})$ \\
\hline 1 & BB $S=1(\tan \phi=1 / 3$, AKLT $)$ & $t-\Delta-\bar{J}-V-\mu$ & C - GS \\
\hline 1 & BB $S=1(\phi=7 \pi / 4, \mathrm{~TB})$ & $t-\Delta-\bar{J}-V-\mu$ & $\mathrm{C}-\mathrm{E}(\mathrm{BA})$ \\
\hline 1 & $\mathrm{BB} S=1(\phi=3 \pi / 2, \mathrm{~K})$ & $\Delta-\bar{J}-V-\mu$ & C - QE \\
\hline 1 & $S=3 / 2$ & F $(S=1 / 2)$ Hubbard & C - E (BA) \\
\hline 2 & $U(1)$ gauge magnet & F strings & $C-E$ \\
\hline 2 & Shastry-Sutherland $(S=1 / 2)$ & $t-t^{\prime}-V-V^{\prime}$ & C - GS \\
\hline Any & BB $S=1(\phi=5 \pi / 4)$ & $t-\bar{J}-V-\mu$ & $C-Q E$ \\
\hline Any & Anisotropic $S=1$ Heisenberg & B $(S=1 / 2)$ Hubbard & $\mathrm{P}-\mathrm{U}$ \\
\hline
\end{tabular}

TABLE I: Equivalence between spin models (model A) and particle models (model B), equivalence that can be simply established by using our fundamental theorem. The meaning of the different terms is clarified in the text (BB: bilinear-biquadratic, F: fermionic, $\mathrm{B}$ : bosonic). Last column describes the type of equivalence (C: complete Hilbert space mapping, P: partial Hilbert space mapping) and type of solutions (E: exact, E (BA): exact Bethe-ansatz, GS: only ground state, QE: quasi-exact, U: unsolvable).

Hamiltonian in an invariant subspace can always be described by a class of languages which are more basic than the original one. By more basic we mean a lower dimension of the local Hilbert space, or in other words, languages describing systems with less degrees of freedom. There are particular cases where the simplification is even deeper, since by using a more basic language we also recognize hidden symmetries for the restricted action of the Hamiltonian in the invariant subspace. The quasi-exact solution of the $1 d t-J_{z}[6]$ model, is an example of successful application of this concept. This quasi-exact solution led to the exact quantum phase diagram and charge excitations of the $t-J_{z}$ model [6].

\section{A. $S U(2)$ Heisenberg Magnets}

The $X Y Z S=1 / 2$ spin model $(D=2)$ is the most popular example of a family of Hamiltonians which is Bethe-ansatz solvable in $d=1$ [47]

$$
H_{\mathrm{xyz}}=\sum_{\langle\mathbf{i}, \mathbf{j}\rangle} J_{x} S_{\mathbf{i}}^{x} S_{\mathbf{j}}^{x}+J_{y} S_{\mathbf{i}}^{y} S_{\mathbf{j}}^{y}+J_{z} S_{\mathbf{i}}^{z} S_{\mathbf{j}}^{z}
$$

Using the MM [4] transformation of Eq. [8, the $X Y Z$ model is mapped onto a gas of interacting hard-core bosons with density-density interactions and particle non-conserving

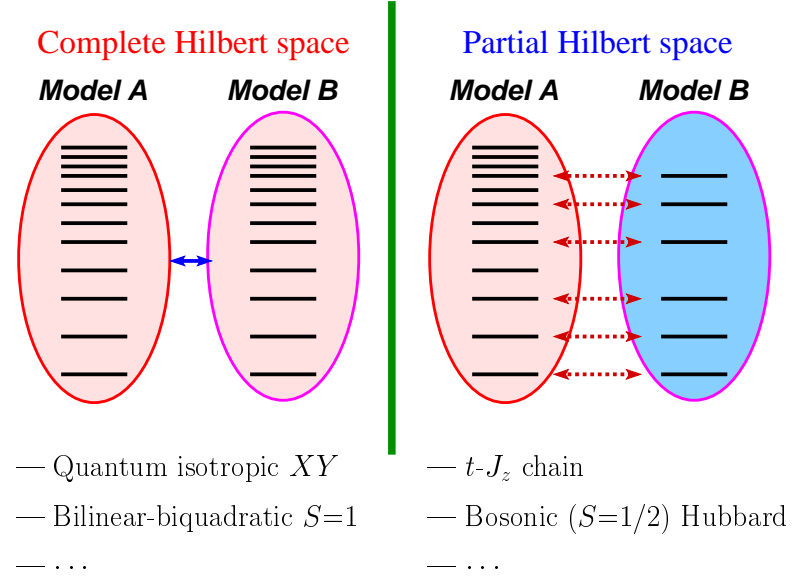

FIG. 6: Schematics of the two types of mappings that one can perform: Complete and partial Hilbert space mappings. The figure also shows examples illustrating each class.

terms

$$
\begin{aligned}
H_{\mathrm{xyz}} & =t \sum_{\langle\mathbf{i}, \mathbf{j}\rangle}\left(\bar{b}_{\mathbf{i}}^{\dagger} \bar{b}_{\mathbf{j}}+\bar{b}_{\mathbf{j}}^{\dagger} \bar{b}_{\mathbf{i}}\right)+\Delta \sum_{\langle\mathbf{i}, \mathbf{j}\rangle}\left(\bar{b}_{\mathbf{i}}^{\dagger} \bar{b}_{\mathbf{j}}^{\dagger}+\bar{b}_{\mathbf{j}} \bar{b}_{\mathbf{i}}\right) \\
& +V \sum_{\langle\mathbf{i}, \mathbf{j}\rangle}\left(\bar{n}_{\mathbf{i}}-\frac{1}{2}\right)\left(\bar{n}_{\mathbf{j}}-\frac{1}{2}\right)
\end{aligned}
$$

where $t=\left(J_{x}+J_{y}\right) / 4, \Delta=\left(J_{x}-J_{y}\right) / 4$, and $V=J_{z}$. Note that in $d=1$ the non-local transmutator is a symmetry and, therefore, we could have used spinless fermions instead of hard-core bosons in Eq. 82.

Another family of spin Hamiltonians $(D=3)$ which has been extensively studied is the bilinear-biquadratic (BB) $S=1$ Heisenberg model $(J>0)$

$$
H_{\phi}=J \sqrt{2} \sum_{\langle\mathbf{i}, \mathbf{j}\rangle}\left[\cos \phi \mathbf{S}_{\mathbf{i}} \cdot \mathbf{S}_{\mathbf{j}}+\sin \phi\left(\mathbf{S}_{\mathbf{i}} \cdot \mathbf{S}_{\mathbf{j}}\right)^{2}\right] .
$$

Using the $S=1$ generalization of the JW transformation for hard-core bosons [22],

$$
\begin{aligned}
S_{\mathbf{j}}^{+} & =\sqrt{2}\left(\bar{b}_{\mathbf{j} \uparrow}^{\dagger}+\bar{b}_{\mathbf{j} \downarrow}\right) \\
S_{\mathbf{j}}^{-} & =\sqrt{2}\left(\bar{b}_{\mathbf{j} \uparrow}+\bar{b}_{\mathbf{j} \downarrow}^{\dagger}\right) \\
S_{\mathbf{j}}^{z} & =\bar{n}_{\mathbf{j} \uparrow}-\bar{n}_{\mathbf{j} \downarrow}
\end{aligned}
$$

(see section $\mathrm{VC}$, $H_{\phi}$ is mapped onto a $t$ - $J$-like model for $S=1 / 2$ hard-core bosons including particle non-conserving terms

$$
\begin{array}{r}
H_{\phi}=\sum_{\langle\mathbf{i}, \mathbf{j}\rangle, \sigma}\left(t \bar{b}_{\mathbf{i} \sigma}^{\dagger} \bar{b}_{\mathbf{j} \sigma}+\Delta \bar{b}_{\mathbf{i} \sigma}^{\dagger} \bar{b}_{\mathbf{j} \sigma}^{\dagger}+\text { H.c. }\right)+4 \Delta \sum_{\langle\mathbf{i}, \mathbf{j}\rangle} s_{\mathbf{i}}^{z} \cdot s_{\mathbf{j}}^{z} \\
+\bar{J} \sum_{\langle\mathbf{i}, \mathbf{j}\rangle}\left(\mathbf{s}_{\mathbf{i}} \cdot \mathbf{s}_{\mathbf{j}}-\frac{\bar{n}_{\mathbf{i}} \bar{n}_{\mathbf{j}}}{4}\right)+V \sum_{\langle\mathbf{i}, \mathbf{j}\rangle} \bar{n}_{\mathbf{i}} \bar{n}_{\mathbf{j}}-\mu \sum_{\mathbf{j}}\left(\bar{n}_{\mathbf{j}}-1\right),(85
\end{array}
$$

where $\mathbf{s}_{\mathbf{j}}=\frac{1}{2} \bar{b}_{\mathbf{j} \alpha}^{\dagger} \boldsymbol{\sigma}_{\alpha \beta} \bar{b}_{\mathbf{j} \beta}$ ( $\boldsymbol{\sigma}$ denoting Pauli matrices), $t=$ $J \sqrt{2} \cos \phi, \Delta=J \sqrt{2}(\cos \phi-\sin \phi), \bar{J}=J 2 \sqrt{2} \sin \phi, V=$ 
$\bar{J}, \mu=z J \sqrt{2} \sin \phi$, and $\mathrm{z}$ is the coordination of the lattice. Again, for $d=1$ the exchange statistics of the particles is irrelevant and one could have used $S=1 / 2$ constrained fermions instead of hard-core bosons. From the known solutions in the spin model one can immediately recognize the solvable cases in the particle model Eq. 85. These are: $\phi=\pi / 4$ $(d=1)$ Lai-Sutherland (LS) [48], $\phi=7 \pi / 4(d=1)$ TakhtajanBabujian (TB) [49], $\tan \phi=1 / 3(d=1)$ Affleck-KennedyLieb-Tasaki (AKLT) [50], $\phi=3 \pi / 2(d=1)$ Klümper (K) [51], and $\phi=5 \pi / 4$ (any $d$ ) our work in Ref. [46].

Of particular current interest are the $S=1 / 2$ Heisenberg models on ladders. The simplest case corresponds to having only nearest-neighbor magnetic interactions (see Fig. (7) )

$$
\begin{aligned}
& H_{\text {Heis }}^{\text {ladd }}=J_{1} \sum_{\mathbf{j}}\left(\Delta_{1} S_{\mathbf{j} 1}^{z} S_{\mathbf{j} 2}^{z}+S_{\mathbf{j} 1}^{x} S_{\mathbf{j} 2}^{x}+S_{\mathbf{j} 1}^{y} S_{\mathbf{j} 2}^{y}\right) \\
& +J_{2} \sum_{\mathbf{j}, \nu=1,2}\left(\Delta_{2} S_{\mathbf{j} \nu}^{z} S_{\mathbf{j}+1 \nu}^{z}+S_{\mathbf{j} \nu}^{x} S_{\mathbf{j}+1 \nu}^{x}+S_{\mathbf{j} \nu}^{y} S_{\mathbf{j}+1 \nu}^{y}\right) .
\end{aligned}
$$

Using the mapping given in Eqs. 42 which connects $S=1 / 2$ spins with canonical fermions, $H_{\text {Heis }}^{\text {ladd }}$ can be rewritten as a Hubbard-like model on a linear chain (up to an irrelevant constant). For instance, if $\Delta_{1}=1$ and $\Delta_{2}=0$ we get

$$
\begin{aligned}
& H_{\text {Heis }}^{\text {ladd }}=t \sum_{\mathbf{j}, \sigma} c_{\mathbf{j} \sigma}^{\dagger} c_{\mathbf{j}+1 \bar{\sigma}}^{\dagger}\left(1-\hat{n}_{\mathbf{j} \bar{\sigma}}-\hat{n}_{\mathbf{j}+1 \sigma}\right)+\text { H.c. } \\
& \quad+t \sum_{\mathbf{j}, \sigma} c_{\mathbf{j} \sigma}^{\dagger} c_{\mathbf{j}+1 \sigma}\left[\left(1-\hat{n}_{\mathbf{j} \bar{\sigma}}\right)\left(1-\hat{n}_{\mathbf{j}+1 \bar{\sigma}}\right)-\hat{n}_{\mathbf{j} \bar{\sigma}} \hat{n}_{\mathbf{j}+1 \bar{\sigma}}\right]+\text { H.c. } \\
& \quad-U \sum_{\mathbf{j}} \hat{n}_{\mathbf{j} \uparrow} \hat{n}_{\mathbf{j} \downarrow}
\end{aligned}
$$

where $U=J_{1}$ and $t=J_{2}$. This is a correlated Hubbard model with zero two-body hopping terms plus a superconducting term. In the absence of the superconducting term, this model has been exactly solved by Arrachea and Aligia [52]. To eliminate the superconducting terms the original spin model has to be modified in the following way

$$
\begin{aligned}
& \tilde{H}_{\text {Heis }}^{\text {ladd }}=J_{1} \sum_{\mathbf{j}}\left(\Delta_{1} S_{\mathbf{j} 1}^{z} S_{\mathbf{j} 2}^{z}+S_{\mathbf{j} 1}^{x} S_{\mathbf{j} 2}^{x}+S_{\mathbf{j} 1}^{y} S_{\mathbf{j} 2}^{y}\right) \\
& \quad+J_{2} \sum_{\mathbf{j}, \nu=1,2} P_{\mathbf{j}}\left(\Delta_{2} S_{\mathbf{j} \nu}^{z} S_{\mathbf{j}+1 \nu}^{z}+S_{\mathbf{j} \nu}^{x} S_{\mathbf{j}+1 \nu}^{x}+S_{\mathbf{j} \nu}^{y} S_{\mathbf{j}+1 \nu}^{y}\right) P_{\mathbf{j}},
\end{aligned}
$$

where $P_{\mathbf{j}}=\sum_{\nu=1,2}\left(S_{\mathbf{j} \nu}^{z}+S_{\mathbf{j}+1 \nu}^{z}\right)=\hat{n}_{\mathbf{j} \uparrow}-\hat{n}_{\mathbf{j} \downarrow}+\hat{n}_{\mathbf{j}+1 \uparrow}-$ $\hat{n}_{\mathbf{j}+1 \downarrow}=2\left(s_{\mathbf{j}}^{z}+s_{\mathbf{j}+1}^{z}\right)$. $\tilde{H}_{\text {Heis }}^{\text {ladd }}$ for $\Delta_{1}=1$ and $\Delta_{2}=0$ is equivalent to the Hubbard model with correlated hopping solved in Ref. [52]. If the sites 1 and 2 are interchanged in one sublattice (see Fig.7 7 ), the sign of the three-body hoppings changes from positive to negative.

Let us consider now the Heisenberg spin-ladder model illustrated in Fig. 8

$$
\begin{aligned}
& H_{\mathrm{SS}}=J_{1} \sum_{\mathbf{j}}\left(\Delta_{1} S_{\mathbf{j} 1}^{z} S_{\mathbf{j} 2}^{z}+S_{\mathbf{j} 1}^{x} S_{\mathbf{j} 2}^{x}+S_{\mathbf{j} 1}^{y} S_{\mathbf{j} 2}^{y}\right) \\
& +J_{2} \sum_{\mathbf{j}, \nu, \nu^{\prime}}\left(\Delta_{2} S_{\mathbf{j} \nu}^{z} S_{\mathbf{j}+1 \nu^{\prime}}^{z}+S_{\mathbf{j} \nu}^{x} S_{\mathbf{j}+1 \nu^{\prime}}^{x}+S_{\mathbf{j} \nu}^{y} S_{\mathbf{j}+1 \nu^{\prime}}^{y}\right) .
\end{aligned}
$$
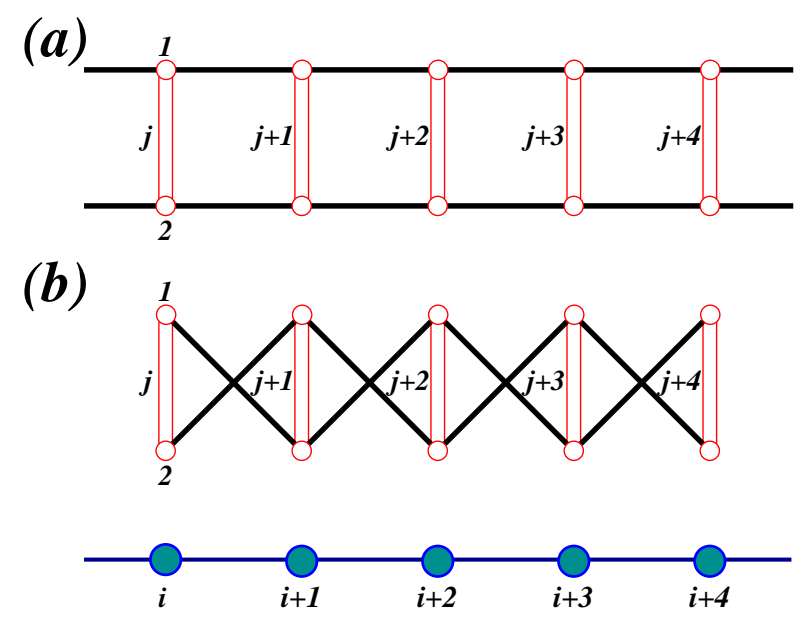

FIG. 7: A spin $S=1 / 2$ Heisenberg ladder mapped onto a Hubbard chain model with correlated hopping. Models $a$ and $b$ are related through a gauge transformation.

The isotropic limit $\left(\Delta_{1}=\Delta_{2}=1\right)$ of this model has been considered by Sutherland [53] who established that for $J_{1}>$ $2 J_{2}$ the ground state is a product state of bond singlets. Again, using Eqs. 42, $H_{\mathrm{SS}}$ can be rewritten as a $1 d$ Hubbard-like model (up to an irrelevant constant)

$$
\begin{aligned}
& H_{\mathrm{SS}}=t \sum_{\mathbf{j}, \sigma} c_{\mathbf{j} \sigma}^{\dagger} c_{\mathbf{j}+1 \bar{\sigma}}^{\dagger}\left(1-\hat{n}_{\mathbf{j} \bar{\sigma}}\right)\left(1-\hat{n}_{\mathbf{j}+1 \sigma}\right)+\text { H.c. } \\
& +t \sum_{\mathbf{j}, \sigma} c_{\mathbf{j} \sigma}^{\dagger} c_{\mathbf{j}+1 \sigma}\left(1-\hat{n}_{\mathbf{j} \bar{\sigma}}\right)\left(1-\hat{n}_{\mathbf{j}+1 \bar{\sigma}}\right)+\text { H.c. } \\
& +J_{z} \sum_{\mathbf{j}} s_{\mathbf{j}}^{z} s_{\mathbf{j}+1}^{z}-U \sum_{\mathbf{j}} \hat{n}_{\mathbf{j} \uparrow} \hat{n}_{\mathbf{j} \downarrow}-\mu \sum_{\mathbf{j}} \hat{n}_{\mathbf{j}},
\end{aligned}
$$

where $U=J_{1} \Delta_{1}, t=J_{2}, J_{z}=4 J_{2} \Delta_{2}$, and $\mu=$ $\left(1-\Delta_{1}\right) J_{1} / 2$. This model is quasi-exactly solvable in the isotropic case $\Delta_{1}=\Delta_{2}=1$. In addition, if we cancel the superconducting terms, the resulting model is also quasi-exactly solvable because there are invariant subspaces for which the action of the Hamiltonian can be mapped onto a $t-J_{z}$ chain model [6] (remember that a constrained $S=1 / 2$ fermion is related to the canonical ones through the relation $\left.\bar{c}_{\mathbf{j} \sigma}^{\dagger}=c_{\mathbf{j} \sigma}^{\dagger}\left(1-\hat{n}_{\mathbf{j} \bar{\sigma}}\right)\right)$.

For correlated hopping model Hamiltonians, the previous mapping, Eq. 42, works well. However, for standard (noncorrelated) Hamiltonians it does not. Let us introduce a new transformation that is expected to work in the latter case

$$
\left\{\begin{array}{l}
S_{\mathbf{j} 1}^{+}=c_{\mathbf{j} \uparrow}^{\dagger} \bar{K}_{\mathbf{j} \uparrow}, \\
S_{\mathbf{j} 1}^{z}=\hat{n}_{\mathbf{j} \uparrow}-\frac{1}{2}, \\
S_{\mathbf{j} 2}^{+}=c_{\mathbf{j} \downarrow}^{\dagger} \bar{K}_{\mathbf{j} \downarrow}, \\
S_{\mathbf{j} 2}^{z}=\hat{n}_{\mathbf{j} \downarrow}-\frac{1}{2},
\end{array}\right.
$$




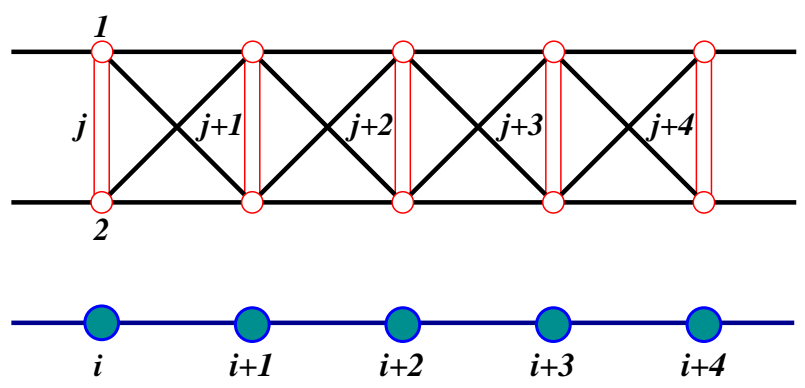

FIG. 8: Sutherland $S=1 / 2$ model and its mapping onto a Hubbardlike chain model.

where the nonlocal operator $\bar{K}_{\mathbf{j} \sigma}$ is now defined as

$$
\begin{aligned}
\bar{K}_{\mathbf{j} \uparrow} & =\exp \left[i \pi\left(\sum_{\mathbf{l}} \hat{n}_{\mathbf{l} \downarrow}+\sum_{\mathbf{1}<\mathbf{j}} \hat{n}_{\mathbf{l} \uparrow}\right)\right], \\
\bar{K}_{\mathbf{j} \downarrow} & =\exp \left[i \pi \sum_{\mathbf{1}<\mathbf{j}} \hat{n}_{\mathbf{l} \downarrow}\right] .
\end{aligned}
$$

This transformation was originally introduced by Mattis and Nam [54] to solve the following Hubbard-like model

$$
\begin{array}{r}
H_{\mathrm{MN}}=\frac{\epsilon}{4} \sum_{\mathbf{j}, \sigma}^{N-1}\left(c_{\mathbf{j} \sigma}^{\dagger}-c_{\mathbf{j} \sigma}\right)\left(c_{\mathbf{j}+1 \sigma}^{\dagger}+c_{\mathbf{j}+1 \sigma}\right) \\
+U \sum_{\mathbf{j}=1}^{N}\left(\hat{n}_{\mathbf{j} \uparrow}-\frac{1}{2}\right)\left(\hat{n}_{\mathbf{j} \downarrow}-\frac{1}{2}\right),
\end{array}
$$

which after the spin-particle transformation, Eq. 90, becomes $(\nu=1,2)$

$$
H_{\mathrm{MN}}=\epsilon \sum_{\mathbf{j}, \nu}^{N-1} S_{\mathbf{j} \nu}^{x} S_{\mathbf{j}+1 \nu}^{x}+U \sum_{\mathbf{j}=1}^{N} S_{\mathbf{j} 1}^{z} S_{\mathbf{j} 2}^{z} .
$$

Using the same transformation, the one-dimensional Hubbard Hamiltonian

$H_{\text {Hubb }}^{1 d}=t \sum_{\mathbf{j}, \sigma}^{N-1}\left(c_{\mathbf{j} \sigma}^{\dagger} c_{\mathbf{j}+1 \sigma}+c_{\mathbf{j}+1 \sigma}^{\dagger} c_{\mathbf{j} \sigma}\right)+U \sum_{\mathbf{j}=1}^{N}\left(\hat{n}_{\mathbf{j} \uparrow}-\frac{1}{2}\right)\left(\hat{n}_{\mathbf{j} \downarrow}-\frac{1}{2}\right)$,

can be transformed into

$$
H_{\text {Hubb }}^{1 d}=2 t \sum_{\mathbf{j}, \nu}^{N-1}\left(S_{\mathbf{j} \nu}^{x} S_{\mathbf{j}+1 \nu}^{x}+S_{\mathbf{j} \nu}^{y} S_{\mathbf{j}+1 \nu}^{y}\right)+U \sum_{\mathbf{j}=1}^{N} S_{\mathbf{j} 1}^{z} S_{\mathbf{j} 2}^{z},
$$

which represents a two-leg ladder made out of two $X Y$-chains coupled by an Ising interaction.

Other interesting examples of quasi-exactly solvable models in $2 d$ of relevance for strongly correlated matter will be presented in a separate publication [55].

\section{B. Single-band fermionic Hubbard Model}

The Hubbard model is the most popular model of a strongly interacting system in condensed matter physics. It contains a kinetic energy term represented by a hopping integral $t$ plus a local on-site Coulomb repulsion $U$. The single-band Hubbard Hamiltonian is $(\sigma=\uparrow, \downarrow)$

$H_{\text {Hubb }}=t \sum_{\langle\mathbf{i}, \mathbf{j}\rangle, \sigma}\left(c_{\mathbf{i} \sigma}^{\dagger} c_{\mathbf{j} \sigma}+c_{\mathbf{j} \sigma}^{\dagger} c_{\mathbf{i} \sigma}\right)+U \sum_{\mathbf{i}}\left(\hat{n}_{\mathbf{i} \uparrow}-\frac{1}{2}\right)\left(\hat{n}_{\mathbf{i} \downarrow}-\frac{1}{2}\right)$.

The dimension of the local Hilbert space is $D=4$ since per lattice site $\mathbf{j}$ we can only have the states: $\left\{|0\rangle, c_{\mathbf{j} \uparrow}^{\dagger}|0\rangle, c_{\mathbf{j} \downarrow}^{\dagger}|0\rangle, c_{\mathbf{j} \uparrow}^{\dagger} c_{\mathbf{j} \downarrow}^{\dagger}|0\rangle\right\}$, and $|0\rangle$ is the vacuum state. Replacing the $c$-operators by the expression for transmutation of statistics given in Eq. 15, we get

$$
\begin{aligned}
H_{\text {Hubb }} & =t \sum_{\langle\mathbf{i}, \mathbf{j}\rangle, \sigma}\left(\tilde{b}_{\mathbf{i} \sigma}^{\dagger} \hat{\mathcal{T}}_{\mathbf{i} \sigma} \hat{\mathcal{T}}_{\mathbf{j} \sigma} K_{\mathbf{i}}^{\dagger} K_{\mathbf{j}} \tilde{b}_{\mathbf{j} \sigma}+\text { H.c. }\right) \\
& +U \sum_{\mathbf{i}}\left(\tilde{n}_{\mathbf{i} \uparrow}-\frac{1}{2}\right)\left(\tilde{n}_{\mathbf{i} \downarrow}-\frac{1}{2}\right),
\end{aligned}
$$

where $\hat{\mathcal{T}}_{\mathbf{i} \uparrow}=\exp \left[i \pi \tilde{n}_{\mathbf{i} \downarrow}\right], \hat{\mathcal{T}}_{\mathbf{i} \downarrow}=\mathbb{1}, K_{\mathbf{j}}=\exp \left[i \sum_{\mathbf{l}} a(\mathbf{l}, \mathbf{j}) \tilde{n}_{\mathbf{l}}\right]$, with $\tilde{n}_{\mathbf{j}}=\tilde{n}_{\mathbf{j} \uparrow}+\tilde{n}_{\mathbf{j} \downarrow}$, and we have used the result

$$
\left[\hat{\mathcal{T}}_{\mathbf{j} \sigma}, K_{\mathbf{i}}^{\dagger}\right]=0 .
$$

The (statistical gauge field) vector potential associated to this transmutation of statistics is

$$
A_{\nu}(\mathbf{j})=\pi\left(\tilde{n}_{\mathbf{j} \downarrow}-\tilde{n}_{\mathbf{j}+\mathbf{e}_{\nu} \downarrow}\right)-\sum_{\mathbf{l}}\left[a(\mathbf{l}, \mathbf{j})-a\left(\mathbf{l}, \mathbf{j}+\mathbf{e}_{\nu}\right)\right] \tilde{n}_{\mathbf{l}} .
$$

Using this expression for the vector potential we can rewrite $H_{\text {Hubb }}$ in the following way

$$
\begin{aligned}
H_{\text {Hubb }} & =t \sum_{\langle\mathbf{i}, \mathbf{j}\rangle, \sigma}\left(\tilde{b}_{\mathbf{i} \sigma}^{\dagger} \exp \left[i A_{\nu}(\mathbf{i})\right] \tilde{b}_{\mathbf{j} \sigma}+\text { H.c }\right) \\
& +U \sum_{\mathbf{i}}\left(\tilde{n}_{\mathbf{i} \uparrow}-\frac{1}{2}\right)\left(\tilde{n}_{\mathbf{i} \downarrow}-\frac{1}{2}\right) .
\end{aligned}
$$

In this way we see that the Hubbard Hamiltonian can be written in a bosonic representation but with interactions which are non-local when $d>1$. When $d=1$ the vector potential acts as a correlated hopping term and the interactions become local

$$
\begin{aligned}
H_{\text {Hubb }}^{1 d} & =t \sum_{\mathbf{i}}\left[\tilde{b}_{\mathbf{i} \uparrow}^{\dagger}\left(1-2 \tilde{n}_{\mathbf{i}+1 \downarrow}\right) \tilde{b}_{\mathbf{i}+1 \uparrow}+\tilde{b}_{\mathbf{i} \downarrow}^{\dagger}\left(1-2 \tilde{n}_{\mathbf{i} \uparrow}\right) \tilde{b}_{\mathbf{i}+1 \downarrow}\right. \\
& + \text { H.c }]+U \sum_{\mathbf{i}}\left(\tilde{n}_{\mathbf{i} \uparrow}-\frac{1}{2}\right)\left(\tilde{n}_{\mathbf{i} \downarrow}-\frac{1}{2}\right) .
\end{aligned}
$$

The $S U(2)$-spin $S=3 / 2$ is another physical object for which the dimension of the local Hilbert space is $D=4$. From our fundamental theorem it is possible to write down these constrained bosons $\tilde{b}_{\mathbf{j} \sigma}^{\dagger}$ in terms of spins $S=3 / 2$. In this way, we can find a $S=3 / 2$ representation for the Hubbard model. A possible mapping between these bosons and a spin $S=3 / 2$ is

$$
\begin{aligned}
S_{\mathbf{j}}^{+} & =\sqrt{3} \tilde{b}_{\mathbf{j} \downarrow}^{\dagger}+2 \tilde{b}_{\mathbf{j} \uparrow}^{\dagger} \tilde{b}_{\mathbf{j} \downarrow}, \\
S_{\mathbf{j}}^{-} & =\sqrt{3} \tilde{b}_{\mathbf{j} \downarrow}+2 \tilde{b}_{\mathbf{j} \downarrow}^{\dagger} \tilde{b}_{\mathbf{j} \uparrow}, \\
S_{\mathbf{j}}^{z} & =\frac{1}{2}\left(\tilde{n}_{\mathbf{j} \uparrow}-\tilde{n}_{\mathbf{j} \downarrow}\right)+\frac{3}{2}\left(\tilde{n}_{\mathbf{j} \uparrow}+\tilde{n}_{\mathbf{j} \downarrow}-1\right) .
\end{aligned}
$$


This mapping can be inverted to get the bosonic operators as a function of the spin operators

$$
\begin{aligned}
& \tilde{b}_{\mathbf{j} \uparrow}^{\dagger}=\frac{1}{2 \sqrt{3}}\left(S_{\mathbf{j}}^{+}\right)^{2}, \\
& \tilde{b}_{\mathbf{j} \downarrow}^{\dagger}=\frac{1}{\sqrt{3}} S_{\mathbf{j}}^{+}\left(S_{\mathbf{j}}^{z}+\frac{1}{2}\right)^{2} .
\end{aligned}
$$

Similarly, the expression for the spin $S=3 / 2$ operators in terms of canonical fermions is

$$
\begin{aligned}
S_{\mathbf{j}}^{+} & =\sqrt{3} c_{\mathbf{j} \downarrow}^{\dagger} K_{\mathbf{j}}+2 c_{\mathbf{j} \uparrow}^{\dagger} c_{\mathbf{j} \downarrow}, \\
S_{\mathbf{j}}^{-} & =\sqrt{3} K_{\mathbf{j}}^{\dagger} c_{\mathbf{j} \downarrow}+2 c_{\mathbf{j} \downarrow}^{\dagger} c_{\mathbf{j} \uparrow}, \\
S_{\mathbf{j}}^{z} & =\frac{1}{2}\left(\hat{n}_{\mathbf{j} \uparrow}-\hat{n}_{\mathbf{j} \downarrow}\right)+\frac{3}{2}\left(\hat{n}_{\mathbf{j} \uparrow}+\hat{n}_{\mathbf{j} \downarrow}-1\right) .
\end{aligned}
$$

Again, we can write down the fermionic operators in terms of the spin operators

$$
\begin{aligned}
c_{\mathbf{j} \uparrow}^{\dagger} & =\frac{1}{2 \sqrt{3}}\left(S_{\mathbf{j}}^{+}\right)^{2} \mathcal{K}_{\mathbf{j} \uparrow}^{\dagger} \\
c_{\mathbf{j} \downarrow}^{\dagger} & =\frac{1}{\sqrt{3}} S_{\mathbf{j}}^{+}\left(S_{\mathbf{j}}^{z}+\frac{1}{2}\right)^{2} \mathcal{K}_{\mathbf{j} \downarrow}^{\dagger} .
\end{aligned}
$$

Using these expressions we can write down the Hubbard model in terms of $S=3 / 2$ spins as

$$
\begin{aligned}
H_{\text {Hubb }} & =\frac{t}{3} \sum_{\langle\mathbf{i}, \mathbf{j}\rangle}\left[S_{\mathbf{i}}^{+}\left(S_{\mathbf{i}}^{z}+\frac{1}{2}\right)^{2} \exp \left[i A_{\nu}(\mathbf{i})\right]\left(S_{\mathbf{j}}^{z}+\frac{1}{2}\right)^{2} S_{\mathbf{j}}^{-}\right. \\
& \left.+\frac{\left(S_{\mathbf{i}}^{+}\right)^{2}}{2} \exp \left[i A_{\nu}(\mathbf{i})\right] \frac{\left(S_{\mathbf{j}}^{-}\right)^{2}}{2}+\text { H.c. }\right] \\
& +\frac{U}{4} \sum_{\mathbf{i}}\left[\left(S_{\mathbf{i}}^{z}\right)^{2}-\frac{5}{4}\right]
\end{aligned}
$$

The $S=3 / 2$ representation of the $1 d$ Hubbard model is

$$
\begin{aligned}
H_{\text {Hubb }}^{1 d} & =-\frac{t}{3} \sum_{\mathbf{j}}\left[S_{\mathbf{j}}^{+}\left(S_{\mathbf{j}}^{z}+\frac{1}{2}\right)\left(S_{\mathbf{j}+1}^{z}+\frac{1}{2}\right)^{2} S_{\mathbf{j}+1}^{-}\right. \\
& \left.+\frac{1}{2}\left(S_{\mathbf{j}}^{+}\right)^{2}\left(S_{\mathbf{j}+1}^{z}+1\right)\left(S_{\mathbf{j}+1}^{-}\right)^{2}+\text { H.c. }\right] \\
& +\frac{U}{4} \sum_{\mathbf{j}}\left[\left(S_{\mathbf{j}}^{z}\right)^{2}-\frac{5}{4}\right] .
\end{aligned}
$$

Since the $1 d$ Hubbard model is Bethe ansatz solvable [56], we are providing a new spin $S=3 / 2$ Hamiltonian which is also Bethe ansatz solvable through the isomorphic mapping between the two languages (fermions and spins).

We can also write down the $1 d$ Hubbard model using the hierarchical $S U(4)$ language. To do so, we fist need to find the dictionary connecting the components of an $S U(4)$-spin in the fundamental representation with the creation and annihilation operators of the particle language

$$
\mathcal{S}(\mathbf{j})=\left(\begin{array}{cccc}
\mathcal{S}^{00}(\mathbf{j}) & \tilde{b}_{\mathbf{j} \uparrow}\left(1-\tilde{n}_{\mathbf{j} \downarrow}\right) & \tilde{b}_{\mathbf{j} \downarrow}\left(1-\tilde{n}_{\mathbf{j} \uparrow}\right) & \tilde{b}_{\mathbf{j} \downarrow} \tilde{b}_{\mathbf{j} \uparrow} \\
\left(1-\tilde{n}_{\mathbf{j} \downarrow}\right) \tilde{b}_{\mathbf{j} \uparrow}^{\dagger} & \mathcal{S}^{11}(\mathbf{j}) & \tilde{b}_{\mathbf{j} \uparrow}^{\dagger} \tilde{b}_{\mathbf{j} \downarrow} & \tilde{n}_{\mathbf{j} \uparrow} \tilde{b}_{\mathbf{j} \downarrow} \\
\left(1-\tilde{n}_{\mathbf{j} \uparrow}\right) \tilde{b}_{\mathbf{j} \downarrow}^{\dagger} & \tilde{b}_{\mathbf{j} \downarrow}^{\dagger} \tilde{b}_{\mathbf{j} \uparrow} & \mathcal{S}^{22}(\mathbf{j}) & \tilde{n}_{\mathbf{j} \downarrow} \tilde{b}_{\mathbf{j} \uparrow} \\
\tilde{b}_{\mathbf{j} \uparrow}^{\dagger} \tilde{b}_{\mathbf{j} \downarrow}^{\dagger} & \tilde{b}_{\mathbf{j} \downarrow}^{\dagger} \tilde{n}_{\mathbf{j} \uparrow} & \tilde{b}_{\mathbf{j} \uparrow}^{\dagger} \tilde{n}_{\mathbf{j} \downarrow} & \mathcal{S}^{33}(\mathbf{j})
\end{array}\right),
$$

where $\mathcal{S}^{00}(\mathbf{j})=\left(1-\tilde{n}_{\mathbf{j} \uparrow}\right)\left(1-\tilde{n}_{\mathbf{j} \downarrow}\right)-\frac{1}{4}, \mathcal{S}^{11}(\mathbf{j})=\tilde{n}_{\mathbf{j} \uparrow}(1-$ $\left.\tilde{n}_{\mathbf{j} \downarrow}\right)-\frac{1}{4}, \mathcal{S}^{22}(\mathbf{j})=\tilde{n}_{\mathbf{j} \downarrow}\left(1-\tilde{n}_{\mathbf{j} \uparrow}\right)-\frac{1}{4}$, and $\mathcal{S}^{33}(\mathbf{j})=\tilde{n}_{\mathbf{j} \uparrow} \tilde{n}_{\mathbf{j} \downarrow}-\frac{1}{4}$. For a general $S U(N)$ group there are actually two kinds of spinors: upper and lower. The upper spinors transform according to the conjugate representation. For the particular case of $S U(2)$, the conjugate representation is equivalent to the original one, i.e., the conjugation is equivalent to a similarity transformation. However, in general for $N>2$ the conjugate representation is not equivalent to the original one. Consequently, the $S U(N>2)$ ferromagnetic and antiferromagnetic Heisenberg Hamiltonians are essentially different operators (in the case of $S U(2)$, they just differ by an overall sign) [57]. In the same way we wrote in Eq. 108 the generators of $s u(4)$ in the fundamental representation, we can write down the corresponding expressions for the generators in the conjugate representation

$\tilde{\mathcal{S}}(\mathbf{j})=\left(\begin{array}{cccc}-\tilde{\mathcal{S}}^{00}(\mathbf{j}) & -\tilde{b}_{\mathbf{j} \uparrow} \tilde{n}_{\mathbf{j} \downarrow} & -\tilde{b}_{\mathbf{j} \downarrow} \tilde{n}_{\mathbf{j} \uparrow} & -\tilde{b}_{\mathbf{j} \downarrow} \tilde{b}_{\mathbf{j} \uparrow} \\ -\tilde{n}_{\mathbf{j} \downarrow} \tilde{b}_{\mathbf{j} \uparrow}^{\dagger} & -\tilde{\mathcal{S}}^{11}(\mathbf{j}) & -\tilde{b}_{\mathbf{j} \uparrow}^{\dagger} \tilde{b}_{\mathbf{j} \downarrow} & \left(\tilde{n}_{\mathbf{j} \uparrow}-1\right) \tilde{b}_{\mathbf{j} \downarrow} \\ -\tilde{n}_{\mathbf{j} \uparrow} \tilde{b}_{\mathbf{j} \downarrow}^{\dagger} & -\tilde{b}_{\mathbf{j} \downarrow}^{\dagger} \tilde{b}_{\mathbf{j} \uparrow} & -\tilde{\mathcal{S}}^{22}(\mathbf{j}) & \left(\tilde{n}_{\mathbf{j} \downarrow}-1\right) \tilde{b}_{\mathbf{j} \uparrow} \\ -\tilde{b}_{\mathbf{j} \uparrow}^{\dagger} \tilde{b}_{\mathbf{j} \downarrow}^{\dagger} & \tilde{b}_{\mathbf{j} \downarrow}^{\dagger}\left(\tilde{n}_{\mathbf{j} \uparrow}-1\right) & \tilde{b}_{\mathbf{j} \uparrow}^{\dagger}\left(\tilde{n}_{\mathbf{j} \downarrow}-1\right) & -\tilde{\mathcal{S}}^{33}(\mathbf{j})\end{array}\right)$,

where $\tilde{\mathcal{S}}^{00}(\mathbf{j})=\tilde{n}_{\mathbf{j} \uparrow} \tilde{n}_{\mathbf{j} \downarrow}-\frac{1}{4}, \tilde{\mathcal{S}}^{11}(\mathbf{j})=\tilde{n}_{\mathbf{j} \downarrow}\left(1-\tilde{n}_{\mathbf{j} \uparrow}\right)-\frac{1}{4}$, $\tilde{\mathcal{S}}^{22}(\mathbf{j})=\tilde{n}_{\mathbf{j} \uparrow}\left(1-\tilde{n}_{\mathbf{j} \downarrow}\right)-\frac{1}{4}$, and $\tilde{\mathcal{S}}^{33}(\mathbf{j})=\left(1-\tilde{n}_{\mathbf{j} \uparrow}\right)(1-$ $\left.\tilde{n}_{\mathbf{j} \downarrow}\right)-\frac{1}{4}$.

The expression of the $1 d$ Hubbard model in terms of the $S U(4)$ hierarchical language is $\left(J_{\mu \nu}=J_{\nu \mu}, J_{\mu \nu}^{\prime}=J_{\nu \mu}^{\prime}\right)$

$$
\begin{aligned}
H_{\text {Hubb }}^{1 d} & =\sum_{\mathbf{j}}\left[J_{\mu \nu} \mathcal{S}^{\mu \nu}(\mathbf{j}) \mathcal{S}^{\nu \mu}(\mathbf{j}+1)+J_{\mu \nu}^{\prime} \mathcal{S}^{\mu \nu}(\mathbf{j}) \tilde{\mathcal{S}}^{\nu \mu}(\mathbf{j}+1)\right. \\
& \left.+\frac{U}{2}\left(\mathcal{S}^{00}(\mathbf{j})+\mathcal{S}^{33}(\mathbf{j})\right)\right]
\end{aligned}
$$

where the non-zero magnetic interactions are: $J_{01}=J_{02}=$ $-J_{13}=-J_{23}=t$ and $J_{01}^{\prime}=-J_{02}^{\prime}=-J_{13}^{\prime}=-J_{23}^{\prime}=$ $-t$. We can see from Eq. 1110 that in this representation the $U$ term plays the role of a magnetic field. The case $J_{\mu \nu}^{\prime}=$ 0 corresponds to the Hubbard-like model which was exactly solved by Arrachea and Aligia [52].

\section{Single-band bosonic Hubbard Model}

We consider now a model of hard-core bosons including two degenerate orbitals $\alpha=\{1,2\}$ per site

$$
\begin{aligned}
H & =-t \sum_{\langle\mathbf{i}, \mathbf{j}\rangle, \alpha, \alpha^{\prime}}\left(\tilde{b}_{\mathbf{i} \alpha}^{\dagger} \tilde{b}_{\mathbf{j} \alpha^{\prime}}+\tilde{b}_{\mathbf{j} \alpha^{\prime}}^{\dagger} \tilde{b}_{\mathbf{i} \alpha}\right)+U \sum_{\mathbf{j}} \tilde{n}_{\mathbf{j} 1} \tilde{n}_{\mathbf{j} 2} \\
& +V \sum_{\langle\mathbf{i}, \mathbf{j}\rangle}\left(\tilde{n}_{\mathbf{i}}-1\right)\left(\tilde{n}_{\mathbf{j}}-1\right)-\mu \sum_{\mathbf{j}} \tilde{n}_{\mathbf{j}}
\end{aligned}
$$

where $\tilde{n}_{\mathbf{j} \alpha}=\tilde{b}_{\mathbf{j} \alpha}^{\dagger} \tilde{b}_{\mathbf{j} \alpha}$ and $\tilde{n}_{\mathbf{j}}=\tilde{n}_{\mathbf{j} 1}+\tilde{n}_{\mathbf{j} 2}$. The first term represents a hopping connecting pair of orbitals belonging to nearest-neighbor sites $\langle\mathbf{i}, \mathbf{j}\rangle$. The second is a local Coulomb repulsion term between particles occupying different orbitals of the same site. The third term corresponds to a nearestneighbor density-density repulsion $V$. The dimension of the 
local Hilbert space is $D=4$ since per lattice site $\mathbf{j}$ we can only have the states: $\left\{|0\rangle, \tilde{b}_{\mathbf{j} 1}^{\dagger}|0\rangle, \tilde{b}_{\mathbf{j} 2}^{\dagger}|0\rangle, \tilde{b}_{\mathbf{j} 1}^{\dagger} \tilde{b}_{\mathbf{j} 2}^{\dagger}|0\rangle\right\}$, and $|0\rangle$ is the vacuum state.

As mentioned in section 1 II B the hard-core boson operators in each site generate an $s u(2)$ algebra: $\left\{\tilde{b}_{\mathbf{i} \alpha}^{\dagger}, \tilde{b}_{\mathbf{i} \alpha}, \tilde{n}_{\mathbf{i} \alpha}-\right.$ $1 / 2\}$. The associated representation is the fundamental one, i.e., $S=1 / 2$. Since there are two orbitals per site, the local algebra associated in each site is the direct sum of two $s u(2)$ algebras, and the representation is the direct sum of both $S=1 / 2$ representations. It is well known that by reducing the direct sum we get two irreducible representations: the singlet and the triplet representations. The singlet is associated to the antisymmetric (under the permutation of both orbitals) state

$$
\left|\psi_{\mathbf{i A}}\right\rangle=\frac{1}{\sqrt{2}}\left(\tilde{b}_{\mathbf{i} 1}^{\dagger}-\tilde{b}_{\mathbf{i} 2}^{\dagger}\right)|0\rangle=\tilde{b}_{\mathbf{i A}}^{\dagger}|0\rangle .
$$

The three remaining states, which belong to the triplet representation, are symmetric. These states can be generated by the creation operator

$$
\tilde{b}_{\mathbf{i} S}^{\dagger}=\frac{1}{\sqrt{2}}\left(\tilde{b}_{\mathbf{i} 1}^{\dagger}+\tilde{b}_{\mathbf{i} 2}^{\dagger}\right) .
$$

By applying this operator to the vacuum state $|0\rangle$ one can get a particular basis for the triplet representation

$$
\left\{|0\rangle, \tilde{b}_{\mathbf{i S}}^{\dagger}|0\rangle, \tilde{b}_{\mathbf{i S}}^{\dagger} \tilde{b}_{\mathbf{i S}}^{\dagger}|0\rangle\right\}
$$

The singlet plus the triplet states generate another basis for the local Hilbert space of $H$ with $D=4\left(\tilde{n}_{\mathbf{j}}=\tilde{b}_{\mathbf{j} \mathrm{A}}^{\dagger} \tilde{b}_{\mathbf{j A}}+\tilde{b}_{\mathbf{j} \mathrm{S}}^{\dagger} \tilde{b}_{\mathbf{j} \mathrm{S}}\right)$. If we apply the MM [4] transformation to these hard-core bosons, we get a spin $S=1 / 2$ for each orbital. After that transformation it becomes clear that $\left|\psi_{\mathrm{iA}}\right\rangle$ is the singlet state, while the other three (Eq. 114) are the triplet states with $S_{z}=-1,0,1$. Note that the local algebra satisfied by $\tilde{b}_{\mathrm{iA}}^{\dagger}$ and $\tilde{b}_{\mathbf{i S}}^{\dagger}$ is not the same as the one satisfied by $\tilde{b}_{\mathbf{i} 1}^{\dagger}$ and $\tilde{b}_{\mathbf{i} 2}^{\dagger}$. In particular, $\tilde{b}_{\mathrm{iA}}^{\dagger} \tilde{b}_{\mathrm{iS}}^{\dagger}=\tilde{b}_{\mathrm{iS}}^{\dagger} \tilde{b}_{\mathrm{iA}}^{\dagger}=0$.

Going back to our model, Eq. 111, we immediately notice that the local singlet state $\left|\psi_{\mathrm{iA}}\right\rangle$ is invariant under the application of $H$. In other words, if there is one particle in an antisymmetric state at site $\mathbf{i}$, that local state will be conserved. There is a local $U(1)$ symmetry associated to this conservation

$$
\left|\psi_{\mathbf{i A}}\right\rangle \rightarrow e^{\Phi_{\mathbf{i}}}\left|\psi_{\mathbf{i A}}\right\rangle
$$

which leaves $H$ invariant. Consequently, one can identify invariant subspaces of $H$. These subspaces are classified according to the set of sites $\mathcal{N}_{\mathrm{A}}$ which are in a singlet state. We can now take advantage of the invariance of these subpaces, and project $H$ onto each of them to reduce the effective number of degrees of freedom. The expression for $H$ (up to a constant) restricted to the subspace associated to the set $\mathcal{N}_{\mathrm{A}}$ is

$$
\begin{aligned}
H_{\mathcal{N}_{\mathrm{A}}} & =-2 t \sum_{\overline{\langle\mathbf{i}, \mathbf{j}\rangle}}\left(\tilde{b}_{\mathbf{i S}}^{\dagger} \tilde{b}_{\mathbf{j} \mathbf{S}}+\tilde{b}_{\mathbf{j} \mathbf{S}}^{\dagger} \tilde{b}_{\mathbf{i S}}\right)-\mu \sum_{\overline{\mathbf{j}}} \tilde{n}_{\mathbf{j} \mathrm{S}} \\
& +V \sum_{\overline{\langle\mathbf{i}, \mathbf{j}\rangle}}\left(\tilde{n}_{\mathbf{i} \mathbf{S}}-1\right)\left(\tilde{n}_{\mathbf{j} \mathbf{S}}-1\right) \\
& +\frac{U}{2} \sum_{\overline{\mathbf{j}}} \tilde{n}_{\mathbf{j} \mathrm{S}}\left(\tilde{n}_{\mathbf{j} \mathrm{S}}-1\right),
\end{aligned}
$$

where $\overline{\langle\mathbf{i}, \mathbf{j}\rangle}$ and $\overline{\mathbf{j}}$ means sites $\mathbf{i}, \mathbf{j} \notin \mathcal{N}_{\mathrm{A}}$ and $\tilde{n}_{\mathbf{j} S}=1-\left[\tilde{b}_{\mathbf{j} S}, \tilde{b}_{\mathbf{j S}}^{\dagger}\right]$. Having a singlet at a given site $\mathbf{j}$ is equivalent to take the site out of the lattice since that local singlet is frozen at $\mathbf{j}$ (Pauli blocking). The action of $H$ on each invariant subspace can be described by the operators $\tilde{b}_{\mathrm{iS}}^{\dagger}$ and $\tilde{b}_{\mathbf{i S}}$, which are generators of an $s u(2)$ algebra in the $S=1$ (triplet) representation. This is easily seen using the mapping of Eq. 66 after replacing the $\bar{g}_{\mathbf{j}}$ 's by the $\tilde{b}_{\mathbf{j} s}$ 's operators acting only on sites $\mathbf{j} \notin \mathcal{N}_{\mathrm{A}}$

$$
\begin{aligned}
S_{\mathbf{j}}^{+} & =\sqrt{2} \tilde{b}_{\mathbf{j} \mathrm{S}}^{\dagger}, \\
S_{\mathbf{j}}^{-} & =\sqrt{2} \tilde{b}_{\mathbf{j} \mathrm{S}}, \\
S_{\mathbf{j}}^{z} & =\tilde{n}_{\mathbf{j} \mathrm{S}}-1 .
\end{aligned}
$$

A natural consequence of this transformation is the possibility of mapping $H_{\mathcal{N}_{\mathrm{A}}}$ onto an $S=1$ spin Hamiltonian. This is done by inverting Eq. 117

$$
\begin{aligned}
\tilde{b}_{\mathbf{j} \mathrm{S}}^{\dagger} & =\frac{1}{\sqrt{2}} S_{\mathbf{j}}^{+}, \\
\tilde{b}_{\mathbf{j} \mathrm{S}} & =\frac{1}{\sqrt{2}} S_{\mathbf{j}}^{-}, \\
\tilde{n}_{\mathrm{j} \mathrm{S}} & =S_{\mathbf{j}}^{z}+1,
\end{aligned}
$$

and replacing these expressions into Eq. 116 to get

$$
\begin{aligned}
H_{\mathcal{N}_{\mathrm{A}}} & =-t \sum_{\langle\mathbf{i}, \mathbf{j}\rangle}\left(S_{\mathbf{i}}^{+} S_{\mathbf{j}}^{-}+S_{\mathbf{i}}^{-} S_{\mathbf{j}}^{+}\right)+V \sum_{\langle\mathbf{i}, \mathbf{j}\rangle} S_{\mathbf{i}}^{z} S_{\mathbf{j}}^{z} \\
& +\frac{U}{2} \sum_{\overline{\mathbf{j}}}\left(S_{\mathbf{j}}^{z}\right)^{2}+\left(\frac{U}{2}-\mu\right) \sum_{\overline{\mathbf{j}}} S_{\mathbf{j}}^{z}
\end{aligned}
$$

This is an anisotropic Heisenberg model $\left(J_{z}=V\right.$ and $J_{x}=$ $J_{y}=-2 t$ ) with a magnetic field $B_{z}=U / 2-\mu$ applied along the $z$ axis. In addition, there is an easy-plane singleion anisotropy. Therefore, each invariant subspace of $H$ gives rise to an anisotropic Heisenberg model $H_{\mathcal{N}_{\mathrm{A}}}$ acting on a partially depleted lattice (i.e., the sites in $\mathcal{N}_{\mathrm{A}}$ are removed from the lattice). From the variational principle it becomes evident that the lowest energy subspace is the one containing no local singlet states, i.e., $\mathcal{N}_{\mathrm{A}}$ is the empty set $\emptyset$ (the original lattice is not depleted).

We know from our fundamental theorem that there are many other possible languages that may be used to describe the present system. In particular, since $\left(\tilde{b}_{\mathbf{i S}}^{\dagger}\right)^{3}=0$ it is clear that these modes satisfy a generalized Pauli exclusion principle with $p=2$ and, therefore, one can establish a mapping to 
$g$-particles which satisfy the algebra written in Eq. (62). The explicit form for this mapping is

$$
\begin{aligned}
\tilde{b}_{\mathbf{j} S}^{\dagger} & =g_{\mathbf{j}}^{\dagger}\left[1+\left(\frac{1}{\sqrt{2}}-1\right) n_{\mathbf{j}}^{g}\right], \\
\tilde{b}_{\mathbf{j} S} & =\left[1+\left(\frac{1}{\sqrt{2}}-1\right) n_{\mathbf{j}}^{g}\right] g_{\mathbf{j}}, \\
\tilde{n}_{\mathbf{j} S} & =n_{\mathbf{j}}^{g},
\end{aligned}
$$

where $n_{\mathbf{i}}^{g}=g_{\mathbf{j}}^{\dagger} g_{\mathbf{j}}$. Again, by replacing these expressions into Eq. 116, we can write down $H_{\mathcal{N}_{\mathrm{A}}}$ in terms of the $g$-particles as

$$
\begin{aligned}
H_{\mathcal{N}_{\mathrm{A}}} & =-\sum_{\overline{\langle\mathbf{i}, \mathbf{j}\rangle}}\left(g_{\mathbf{i}}^{\dagger} g_{\mathbf{j}}+g_{\mathbf{j}}^{\dagger} g_{\mathbf{i}}\right)\left(h_{1}+h_{2}+h_{3}\right) \\
& +\frac{U}{2} \sum_{\overline{\mathbf{j}}} n_{\mathbf{j}}^{g}\left(n_{\mathbf{j}}^{g}-1\right)+V \sum_{\overline{\langle i, \mathbf{j}\rangle}}\left(n_{\mathbf{i}}^{g}-1\right)\left(n_{\mathbf{j}}^{g}-1\right) \\
& -\mu \sum_{\overline{\mathbf{j}}} n_{\mathbf{j}}^{g}
\end{aligned}
$$

where $h_{1}=t_{1}\left(n_{\mathbf{i}}^{g}+n_{\mathbf{j}}^{g}-2\right)\left(n_{\mathbf{i}}^{g}+n_{\mathbf{j}}^{g}-3\right), h_{2}=-t_{2}\left(n_{\mathbf{i}}^{g}+\right.$ $\left.n_{\mathbf{j}}^{g}-1\right)\left(n_{\mathbf{i}}^{g}+n_{\mathbf{j}}^{g}-3\right)$, and $h_{3}=t_{3}\left(n_{\mathbf{i}}^{g}+n_{\mathbf{j}}^{g}-1\right)\left(n_{\mathbf{i}}^{g}+n_{\mathbf{j}}^{g}-2\right)$ with $t_{1}=t, t_{2}=\sqrt{2} t$, and $t_{3}=t / 2$. The correlated hopping terms values are such that the matrix elements of the three possible hopping processes, illustrated in Fig. 9 are the same.

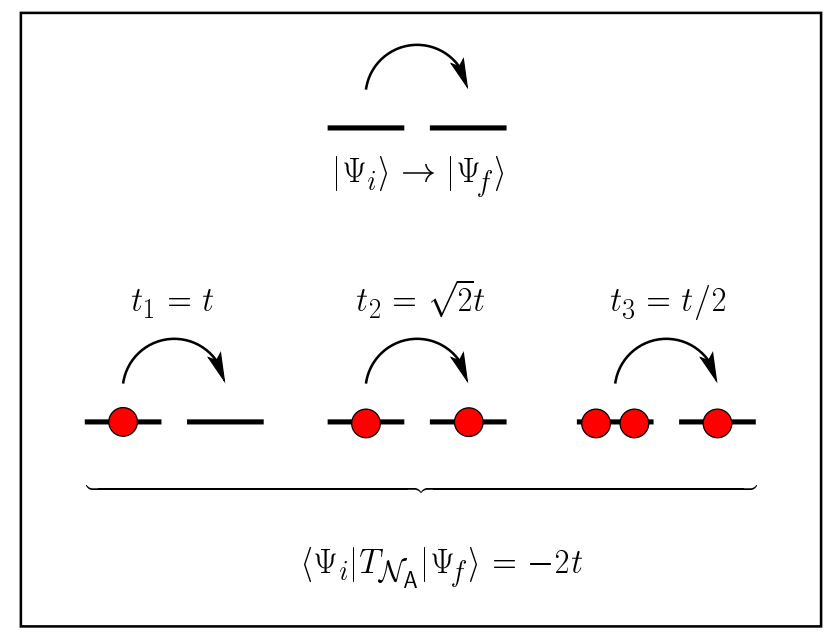

FIG. 9: Hopping processes for the bosonic Hubbard model written in the $g$-particles language, Eq. 121. There are three different hopping processes $t_{1}, t_{2}$, and $t_{3}$. Note, however, that the non-vanishing matrix elements of the kinetic energy $T_{\mathcal{N}_{\mathrm{A}}}$ are the same $(-2 t)$ regardless of the hopping process.

\section{BCS reduced Hamiltonian}

These general mappings between languages are not restricted to real space lattices, indeed one can find mappings between modes in Fourier space, for instance. A very wellknow example is the one introduced by P.W. Anderson [58] for the case of the Bardeen-Cooper-Schrieffer (BCS) reduced Hamiltonian

$$
\begin{aligned}
H_{\mathrm{BCS}} & =\sum_{\mathbf{k}} \epsilon_{\mathbf{k}}\left(\hat{n}_{\mathbf{k} \uparrow}+\hat{n}_{-\mathbf{k} \downarrow}-1\right) \\
& -V \sum_{\mathbf{k}, \mathbf{k}^{\prime}} c_{\mathbf{k}^{\prime} \uparrow}^{\dagger} c_{-\mathbf{k}^{\prime} \downarrow}^{\dagger} c_{-\mathbf{k} \downarrow} c_{\mathbf{k} \uparrow}
\end{aligned}
$$

where the (canonical) fermionic modes (electrons) are defined on a momentum space lattice with momentum index $\mathbf{k}$ and $\hat{n}_{\mathbf{k} \sigma}=c_{\mathbf{k} \sigma}^{\dagger} c_{\mathbf{k} \sigma}$ (with $\left.\sigma=\uparrow, \downarrow\right)$. Again, this is a case where the dimension of the local Hilbert space is $D=4$ with a possible basis $\left\{|0\rangle, c_{\mathbf{k} \uparrow}^{\dagger}|0\rangle, c_{\mathbf{k} \downarrow}^{\dagger}|0\rangle, c_{\mathbf{k} \uparrow}^{\dagger} c_{\mathbf{k} \downarrow}^{\dagger}|0\rangle\right\}$, and it turns out it is exactly solvable.

It is clear from this Hamiltonian that if the total charge of the pair ( $\mathbf{k} \uparrow,-\mathbf{k} \downarrow$ ) is one (i.e., the pair state is occupied by a single electron, $\hat{n}_{\mathbf{k} \uparrow}=1$ and $\hat{n}_{-\mathbf{k} \downarrow}=0$, or $\hat{n}_{\mathbf{k} \uparrow}=0$ and $\hat{n}_{-\mathbf{k} \downarrow}=1$ ) it will remain equal to one after application of $H_{\mathrm{BCS}}$ (Pauli blocking). We will show now that this conservation is derived from an $S U(2)$ gauge symmetry of the BCS reduced Hamiltonian which becomes explicit when we write $H_{\mathrm{BCS}}$ in terms of the generators of $s u(2) \bigoplus s u(2)$. This constitutes another example of the use of alternative languages to unveil hidden symmetries and, to our knowledge, this has not been revealed in the way we will present below.

We just need to note that the following sets of operators

$$
\begin{aligned}
\tau_{\mathbf{k}}^{z} & =\frac{1}{2}\left(\hat{n}_{\mathbf{k} \uparrow}+\hat{n}_{-\mathbf{k} \downarrow}-1\right), \\
\tau_{\mathbf{k}}^{+} & =c_{\mathbf{k} \uparrow}^{\dagger} c_{-\mathbf{k} \downarrow}^{\dagger}, \\
\tau_{\mathbf{k}}^{-} & =c_{-\mathbf{k} \downarrow} c_{\mathbf{k} \uparrow},
\end{aligned}
$$

and

$$
\begin{aligned}
S_{\mathbf{k}}^{z} & =\frac{1}{2}\left(\hat{n}_{\mathbf{k} \uparrow}-\hat{n}_{-\mathbf{k} \downarrow}\right), \\
S_{\mathbf{k}}^{+} & =c_{\mathbf{k} \uparrow}^{\dagger} c_{-\mathbf{k} \downarrow}, \\
S_{\mathbf{k}}^{-} & =c_{-\mathbf{k} \downarrow}^{\dagger} c_{\mathbf{k} \uparrow},
\end{aligned}
$$

satisfy the spin $s u(2)$ commutation relations $(\mu, \nu, \lambda=$ $x, y, z)$

$$
\begin{aligned}
{\left[S_{\mathbf{k}}^{\mu}, S_{\mathbf{k}^{\prime}}^{\nu}\right] } & =i \delta_{\mathbf{k k}^{\prime}} \epsilon_{\mu \nu \lambda} S_{\mathbf{k}}^{\lambda} \\
{\left[\tau_{\mathbf{k}}^{\mu}, \tau_{\mathbf{k}^{\prime}}^{\nu}\right] } & =i \delta_{\mathbf{k k}^{\prime}} \epsilon_{\mu \nu \lambda} \tau_{\mathbf{k}}^{\lambda} \\
{\left[S_{\mathbf{k}}^{\mu}, \tau_{\mathbf{k}^{\prime}}^{\nu}\right] } & =0
\end{aligned}
$$

The last set of local $s u(2)$ generators is associated to the conservation of the parity of the total charge in each pair $(\mathbf{k} \uparrow,-\mathbf{k} \downarrow)$. Since these are local transformations, the symmetry generated by them is an $S U(2)$ gauge symmetry. To see this we just need to re-write $H_{\mathrm{BCS}}$ in the pseudospin language [58]

$$
H_{\mathrm{BCS}}=2 \sum_{\mathbf{k}} \epsilon_{\mathbf{k}} \tau_{\mathbf{k}}^{z}-V \sum_{\mathbf{k}, \mathbf{k}^{\prime}}^{\prime}\left(\tau_{\mathbf{k}^{\prime}}^{x} \tau_{\mathbf{k}}^{x}+\tau_{\mathbf{k}^{\prime}}^{y} \tau_{\mathbf{k}}^{y}\right) .
$$


Since $H_{\mathrm{BCS}}$ is only a function of the set of local $s u(2)$ generators given by Eq. (123) it commutes with the set of generators given by Eq. (124).

In this way, this $S U(2)$ gauge symmetry splits the total Hilbert space into invariant subspaces labelled by the set of momenta $\mathcal{N}_{\text {bp }}$ which have single occupancy of electrons. Within each subspace (which corresponds to a depleted momentum space lattice) the operators $\bar{b}_{\mathbf{k}}^{\dagger}=c_{\mathbf{k} \uparrow}^{\dagger} c_{-\mathbf{k} \downarrow}^{\dagger}$ are the single-flavor hard-core bosons whose algebra was defined in Eq. [6, and the Hamiltonian becomes

$$
H_{\mathrm{BCS}}^{\mathcal{N}_{\mathrm{bp}}}=2 \sum_{\overline{\mathbf{k}}} \epsilon_{\mathbf{k}} \bar{b}_{\mathbf{k}}^{\dagger} \bar{b}_{\mathbf{k}}-V \sum_{\overline{\mathbf{k}}, \overline{\mathbf{k}^{\prime}}}^{\prime} \bar{b}_{\mathbf{k}^{\prime}}^{\dagger} \bar{b}_{\mathbf{k}}
$$

where $\overline{\mathbf{k}}$ and $\overline{\mathbf{k}^{\prime}}$ mean sites in a momentum lattice $\mathbf{k}, \mathbf{k}^{\prime} \notin \mathcal{N}_{\mathrm{bp}}$. (This model is exactly solvable.) Thus, a superconducting BCS state is a Bose-Einstein condensate, in momentum space, of hard-core bosons (i.e., spinless JW particles).

\section{E. Bosonic $t$ - $J$ model}

In the previous section $\mathrm{VA}$ we introduced an $s u(N)$ language for the hard-core bosons (see Eq. 48). In other words, we showed that the generators of $s u(N)$ in the fundamental representation can be used to describe a system of hard-core bosons with $N_{f}=N-1$ different flavors. To illustrate a possible application of this result, we will consider here the case of spin $S=1 / 2$ hard-core bosons (i.e., $N_{f}=2$ and $D=N=3$ ). In particular, we will show that the bosonic $t-J$ model [59]

$$
\begin{aligned}
H_{t-J}^{b} & =t \sum_{\langle\mathbf{i}, \mathbf{j}\rangle, \sigma}\left(\bar{b}_{\mathbf{i} \sigma}^{\dagger} \bar{b}_{\mathbf{j} \sigma}+\bar{b}_{\mathbf{j} \sigma}^{\dagger} \bar{b}_{\mathbf{i} \sigma}\right)+J \sum_{\langle\mathbf{i}, \mathbf{j}\rangle}\left(\mathbf{s}_{\mathbf{i}} \cdot \mathbf{s}_{\mathbf{j}}-\frac{\bar{n}_{\mathbf{i}} \bar{n}_{\mathbf{j}}}{4}\right) \\
& -\mu \sum_{\mathbf{j}} \bar{n}_{\mathbf{j}}
\end{aligned}
$$

with $\mathbf{s}_{\mathbf{j}}=\frac{1}{2} \bar{b}_{\mathbf{j} \alpha}^{\dagger} \boldsymbol{\sigma}_{\alpha \beta} \bar{b}_{\mathbf{j} \beta}(\boldsymbol{\sigma}$ denoting Pauli matrices $)$, can be rewritten as an anisotropic $S U(3)$ Heisenberg model. To this end we just need to use the identities given in Eq. 48 to replace the bosonic operators by the corresponding $s u(3)$ generators $\mathcal{S}^{\mu \nu}(\mathbf{j})$ to get

$$
H_{t-J}^{b}=\sum_{\langle\mathbf{i}, \mathbf{j}\rangle} J_{\mu \nu} \mathcal{S}^{\mu \nu}(\mathbf{i}) \mathcal{S}^{\nu \mu}(\mathbf{j})+B \sum_{\mathbf{j}} \mathcal{S}^{00}(\mathbf{j})
$$

with $\left(J_{\mu \nu}=J_{\nu \mu}\right)$

$$
\begin{aligned}
& J_{00}=-\frac{J}{2}, \quad J_{10}=J_{20}=t, \\
& J_{12}=J_{11}=J_{22}=\frac{J}{2}, \quad B=\mu+\frac{\mathrm{z} J}{6},
\end{aligned}
$$

and $z$ is the coordination of the lattice. In this way we have established an exact mapping between the $t-J$ model and an anisotropic $S U(3)$ Heisenberg model with an applied 'magnetic field' $B$.
We have also seen in section $\nabla \mathrm{A}$ that the $s u(N)$ generators can be represented in terms of Schwinger-Wigner bosons. Therefore, we can use Schwinger-Wigner bosons to get a mean-field solution of the Hamiltonian (Eq. 129). In this way we see how the change of language in the bosonic $t-J$ model opens the possibility to get a simple and original solution [60].

These examples illustrate the process of unveiling hidden connections between different problems just by changing the language. We also saw in the bosonic Hubbard model example that the same process which unveils hidden symmetries reduces the whole space to a direct sum of invariant subspaces. The effective number of degrees of freedom in each subspace is lower than the original one. Consequently, we can use a new class of languages $\mathcal{A} \wedge \Gamma$ with a lower $\operatorname{dim} \Gamma$ to describe the original problem. This reduction not only simplifies the resolution of the problem, but also establishes new connections with other physical systems.

\section{F. $S O(3)$ Heisenberg Model}

As it was shown in Eq. 55, the generators of $s o(3)$ are the components of an antisymmetric tensor of order three. By contracting this tensor with the completely antisymmetric one $\epsilon_{\alpha \mu \nu}$, we can see that the three generators of $s o(3)$ transform like a vector $\mathbf{L}$

$$
L_{\alpha}(\mathbf{j})=\epsilon_{\alpha \mu \nu} \mathcal{M}^{\mu \nu}(\mathbf{j}) .
$$

It is well known that the $s o(3)$ and $s u(2)$ algebras are isomorphic. As a consequence of this, we can establish a simple mapping between $\mathbf{L}$ and the three generators of $s u(2) \mathbf{S}$ in the spin one representation

$$
\mathbf{S}(\mathbf{j})=i \mathbf{L}(\mathbf{j})
$$

Using this basic result of group theory, we can map the $S O(3)$ Heisenberg model

$$
\begin{aligned}
& H_{\text {Heis }}^{\mathrm{SO}(3)}=J \sum_{\langle\mathbf{i}, \mathbf{j}\rangle} \mathbf{L}(\mathbf{i}) \cdot \mathbf{L}(\mathbf{j}) \\
& =-4 J \sum_{\langle\mathbf{i}, \mathbf{j}\rangle} s_{\mathbf{i}}^{y} s_{\mathbf{j}}^{y}+J \sum_{\langle\mathbf{i}, \mathbf{j}\rangle \sigma}\left(\bar{b}_{\mathbf{i} \sigma}^{\dagger} \bar{b}_{\mathbf{j} \sigma}^{\dagger}-\bar{b}_{\mathbf{i} \sigma}^{\dagger} \bar{b}_{\mathbf{j} \sigma}+\text { H.c. }\right)
\end{aligned}
$$

into the $S=1 S U(2)$ Heisenberg model:

$$
H_{\text {Heis }}^{\mathrm{SO}(3)}=-H_{\text {Heis }}^{\mathrm{SU}(2)}=-J \sum_{\langle\mathbf{i}, \mathbf{j}\rangle} \mathbf{S}(\mathbf{i}) \cdot \mathbf{S}(\mathbf{j}) .
$$

In other words, the $S=1 S U(2)$ Heisenberg model is equivalent to a model consisting of a gas of $S=1 / 2$ hard-core bosons with a magnetic interaction along the $y$-axis and a superconducting term.

\section{BROKEN VERSUS EMERGENT SYMMETRY}

Matter organizes in different manners depending upon the nature of its constituents and interactions. Symmetry and 
topology are fundamental guiding principles behind this organization. A symmetry transformation is a modification of the observer's point of view that does not change the outcome of an experiment performed on the same system. In mathematical terms it is a transformation that takes the Hilbert space of states $\mathcal{H}$ into an equivalent one. Wigner's theorem asserts that any transformation $\hat{T}$ which conserves the transition probability between rays in $\mathcal{H}$

$$
\left|\left\langle\hat{T}^{\dagger} \Psi \mid \hat{T} \Phi\right\rangle\right|^{2}=|\langle\Psi \mid \Phi\rangle|^{2}
$$

can be represented by a linear unitary or antilinear antiunitary map $\mathcal{O}\left(\mathcal{O}^{\dagger}=\mathcal{O}^{-1}\right)$ on $\mathcal{H}$. Since the operation of timereversal is one of the few relevant examples in physics which involves an antiunitary operator, we will only consider unitary mappings in the following.

Symmetries may be classified as external or space-time (e.g., the Poincaré group) and internal. The latter refers to the set of transformations that leaves the Hamiltonian of the system $H$ invariant; i.e., these are the symmetries of the physical laws. This set forms a group which is named the internal symmetry group $\mathcal{G}$ and is defined as

$\mathcal{G}=\left\{\mathfrak{g}_{\alpha}\right\}$, with group elements $\mathfrak{g}_{\alpha}$, that satisfy $\left[H, \mathfrak{g}_{\alpha}\right]=0$,

with $\mathfrak{e}$ representing the identity element, and where the number of elements defines its order, which may be finite, denumerable infinite (discrete), or non-denumerable infinite (continuous). In general, groups of symmetries in physics are either finite or Lie groups (non-denumerable infinite). Besides, the group $\mathcal{G}$ may be Abelian (i.e., $\left[\mathfrak{g}_{\alpha}, \mathfrak{g}_{\alpha^{\prime}}\right]=0, \forall \alpha, \alpha^{\prime}$ ) or non-Abelian, and local (also called gauge, meaning that the symmetry applies to subsystems of the original physical system) or global. Invariant physical observables, $O$, are those Hermitian operators which remain invariant under the symmetry group $\mathcal{G}$, i.e., $\left[O, \mathfrak{g}_{\alpha}\right]=0$. Every observable which is a function of the groups elements $\mathfrak{g}_{\alpha}$ is a constant of motion since it commutes with $H$. Table $\coprod$ shows representative examples of physical models displaying different kinds of symmetries. For each group element $\mathfrak{g}_{\alpha}$ there is a unitary operator

\begin{tabular}{|c||c|c|}
\hline Symmetry & Discrete & Continuous \\
\hline \hline \multirow{2}{*}{ Global } & Ising $\left(Z_{2}\right)$ & classical $X Y(O(2))$ \\
& $X Y Z\left(D_{2 h}\right)$ & Heisenberg $(S U(2))$ \\
\hline Local & Gauge magnet $\left(Z_{2}\right)$ & Gauge magnet $(U(1))$ \\
& & BCS (hidden $S U(2))$ \\
\hline
\end{tabular}

TABLE II: Examples of models displaying different kinds of symmetries. The group (or subgroup) of symmetries involved is written in parenthesis.

(see above) that will be denoted as $\mathcal{O}_{\alpha}=\mathcal{O}\left(\mathfrak{g}_{\alpha}\right)$ which maps $\mathcal{H}$ into an equivalent Hilbert space. The set $\left\{\mathcal{O}_{\alpha}\right\}$ forms a representation of the group $\mathcal{G}$. A representation is an homomorphic mapping of the group $\mathcal{G}$ onto a set of linear operators $\mathcal{O}$ such that: $\mathcal{O}(\mathfrak{e})=1$, and $\mathcal{O}_{\alpha} \mathcal{O}_{\beta}=\mathcal{O}\left(\mathfrak{g}_{\alpha} \mathfrak{g}_{\beta}\right)$. The dimension of the representation, $\operatorname{dim}(\mathcal{O})$, is the dimension of the (vector) space on which it acts. By a representation we will mean a non-singular (in particular, unitary) $\operatorname{dim}(\mathcal{O}) \times \operatorname{dim}(\mathcal{O})$ matrix representation. A representation is irreducible if its invariant subspaces under the action of all the elements of the group are only $\mathbf{0}$ and the full space. A completely reducible representation can be written as a direct sum of irreducible representations (irreps). The eigenstates of $H$ that have the same eigenvalue $E_{n}$ form an invariant subspace

$$
H \mathcal{O}_{\alpha}\left|\Psi_{n}\right\rangle=\mathcal{O}_{\alpha} H\left|\Psi_{n}\right\rangle=E_{n} \mathcal{O}_{\alpha}\left|\Psi_{n}\right\rangle
$$

meaning $\left|\Psi_{n}^{\prime}\right\rangle=\mathcal{O}_{\alpha}\left|\Psi_{n}\right\rangle$ is also an eigenstate with the same eigenvalue. When the dimension of this invariant subspace is larger than one, the energy eigenvalue $E_{n}$ is degenerate. The dimension of the degenerate subspace is equal to the dimension of the representation of $\mathcal{G}$ associated with the eigenstate $\left|\Psi_{n}\right\rangle$. If the group $\mathcal{G}$ is Abelian all the irreps are onedimensional and there is no degeneracy induced by $\mathcal{G}$.

Lie groups play a fundamental role in physics. There is a notion of continuity or closeness imposed on the elements of the group manifold $\mathfrak{g}_{\alpha}$ such that a finite transformation of the group can be generated by a series of infinitesimal ones. For a one-parameter continuous group the representations (the homomorphic mapping must be continuous) of its elements can be written

$$
\mathcal{O}_{\alpha}(\theta)=\exp \left[i \theta X_{\alpha}\right]
$$

where $\theta$ is a continuous parameter and $X_{\alpha}$ 's are the generators of the Lie algebra. The representations of the group elements are defined such that $\theta=0$ represents the identity operator $\mathbb{1}$ and an infinitesimal transformation $\delta \theta$ is expressed as

$$
\mathcal{O}_{\alpha}(\delta \theta)=11+i \delta \theta X_{\alpha},
$$

where the generators form a Lie algebra,

$$
\left[X_{\alpha}, X_{\beta}\right]=i C_{\alpha \beta}^{\gamma} X_{\gamma}
$$

with $C_{\alpha \beta}^{\gamma}$ representing the structure constants of the algebra. Notice that the generators themselves are conserved quantities; i.e., $\left[H, X_{\alpha}\right]=0$.

Let us provide an example to show how these ideas are applied. Suppose we have the following model Hamiltonian representing interacting spinless fermions

$$
H=-t \sum_{\langle\mathbf{i}, \mathbf{j}\rangle}\left(c_{\mathbf{i}}^{\dagger} c_{\mathbf{j}}+c_{\mathbf{j}}^{\dagger} c_{\mathbf{i}}\right)+V \sum_{\langle\mathbf{i}, \mathbf{j}\rangle}\left(\hat{n}_{\mathbf{i}}-\frac{1}{2}\right)\left(\hat{n}_{\mathbf{j}}-\frac{1}{2}\right),
$$

where $\langle\mathbf{i}, \mathbf{j}\rangle$ represents nearest neighbors in an otherwise bipartite lattice (i.e., the union of two interpenetrating sublattices $A$ and $B$ ). Among the elements of $\mathcal{G}$, there are two Abelian symmetries: one continuous and global $U(1)$ related to charge conservation, and another discrete and local (staggered) $Z_{2}$ related to a particle-hole transformation. The continuous symmetry is realized by the unitary mappings

$$
\mathcal{O}_{\theta}=\exp \left[i \theta \sum_{\mathbf{j}} \hat{n}_{\mathbf{j}}\right], \quad \mathcal{O}_{\theta} c_{\mathbf{j}}^{\dagger} \mathcal{O}_{\theta}^{\dagger}=\exp [i \theta] c_{\mathbf{j}}^{\dagger},
$$


while the discrete one is realized by the identity and the particle-hole transformation

$$
\begin{aligned}
\mathcal{O}_{\mathrm{p}-\mathrm{h}} & =\prod_{\mathbf{j}} \exp \left[i \pi \delta_{\mathbf{j} B} \hat{n}_{\mathbf{j}}\right] \exp \left[i \frac{\pi}{2}\left(c_{\mathbf{j}}^{\dagger}+c_{\mathbf{j}}\right)\right] \\
\mathcal{O}_{\mathrm{p}-\mathrm{h}} c_{\mathbf{j}}^{\dagger} \mathcal{O}_{\mathrm{p}-\mathrm{h}}^{\dagger} & =\left\{\begin{array}{cc}
c_{\mathbf{j}} & \text { sublattice } A \\
-c_{\mathbf{j}} & \text { sublattice } B
\end{array}\right.
\end{aligned}
$$

where $\delta_{\mathbf{j} B}$ is one if $\mathbf{j}$ belongs to sublattice $B$ and zero otherwise.

In some instances the states of matter display the symmetries compatible with the quantum equations of motion (i.e., symmetries of $H$ )

$$
H|\Psi(t)\rangle=i \partial_{t}|\Psi(t)\rangle
$$

while other more interesting situations are characterized by states with less (broken-symmetry scenario) or with more symmetries (emergent symmetry scenario). In subsection VIIB we will introduce and expand on the latter concept.

\section{A. Broken Symmetry}

To put the concept of emergent symmetry in context let us start summarizing the, in principle independent but very powerful, broken-symmetry scenario. The broken-symmetry phenomenon, which is manifested by a lowest-energy state not having the full symmetry of the Hamiltonian $\mathcal{G}$ but less, has been beautifully described in P. W. Anderson's Basic Notions of Condensed Matter Physics book [8]. Here we will simply restate the main known results.

To say that the ground state of $H,\left|\Psi_{0}\right\rangle$, is invariant under $\mathcal{G}$ means that

$$
\mathcal{O}_{\alpha}\left|\Psi_{0}\right\rangle=\left|\Psi_{0}\right\rangle, \forall \alpha
$$

If $\left|\Psi_{0}\right\rangle$ is not invariant under a given symmetry operation $\mathcal{O}_{\alpha}$, we say that the symmetry is spontaneously broken. (Notice that if such a symmetry does not exist and $\left|\Psi_{0}\right\rangle$ is not invariant that means that the symmetry is explicitly broken.) The broken-symmetry state is also called the ordered state. In general, a subset of $\mathcal{G}, \mathcal{G}_{B S}$, contains the transformations that do not leave $\left|\Psi_{0}\right\rangle$ invariant, while a residual symmetry subgroup $\mathcal{G}_{R}$ remains.

To make a quantitative distinction between the symmetric and the broken-symmetry phases we need to introduce the concept of order parameter, representing the supplementary variable needed to describe the lower symmetry state. By definition, the order parameter is a physical quantity which is zero for the symmetric phase and non-zero for the brokensymmetry state. However, this simple requirement is still too general since there are many different quantities that can satisfy this condition. The most natural choice is dictated by the symmetry which is spontaneously broken. The order parameter is then a physical quantity that transforms like a non-trivial representation of the symmetry group. The adequate choice of representation depends on the particular problem under consideration. For instance, out of a broken $S U(2)$ symmetry, a system may have dipolar (usual magnetic ordering), nematic, or more sophisticated multipolar orderings. Each transforms like a different representation of the $S U(2)$ group. One of the most important experimental challenges is the design of physical measurements that distinguish between the different representations of the broken-symmetry groups.

Spontaneous symmetry breaking can be associated with collective phenomena, which is the relevant case for matter organization, or with a trivial phenomenon that occurs when part of the one-particle spectrum of $H$ is degenerate. The latter scenario is not of interest here because it does not lead to the important concept of ergodicity breaking. As an example, consider the ground state of an odd number of noninteracting spin-1/2 fermions. The ground state is not $S U(2)$ invariant (i.e, it breaks that symmetry) but as soon as we add one particle to the system the $S U(2)$ symmetry is restored. On the other hand, when the phenomenon is collective, spontaneous symmetry breaking is in general related to ergodicity breaking meaning that for given initial conditions the equations of motion, although symmetric, cannot connect (because of kinematic or dynamical reasons) states that would otherwise restore those symmetries. The phenomenon of ergodicity breaking is not exclusive to systems with a macroscopically large number of degrees of freedom (thermodynamic limit), it may happen in systems with a finite number of degrees of freedom as well (e.g., a single particle in a double well with an infinite barrier in between). The first case is a result of the existence of a multitude of inequivalent representations of the observables of an infinite system. In other words, it is a result of the non-commutativity of the limits

$$
\lim _{\Omega \rightarrow \infty} \lim _{F \rightarrow 0} \psi(\Omega, F) \neq \lim _{F \rightarrow 0} \lim _{\Omega \rightarrow \infty} \psi(\Omega, F),
$$

where $\Omega$ is the volume of the system, and $F$ is the generalized external field that linearly couples to the order parameter $\psi$. Notice that when the symmetry breaking is induced by ergodicity breaking, the subspaces which get disconnected in the dynamics of the system are still connected by symmetry operations of $\mathcal{G}_{B S}$. This means that ergodicity breaking in a system with a Hamiltonian invariant under $\mathcal{G}_{B S}$ requires the ground state to be degenerate.

Our last observation raises the following question: Is it possible to have spontaneous symmetry breaking when the ground state is non-degenerate? The answer to this question is yes. Symmetry breaking implies that the ground state is not invariant under the action of $\mathcal{G}_{B S}$. Non-degeneracy means that the representation associated to this state is one-dimensional. Let's consider an example of this situation in which $\mathcal{G}$ is the Abelian group of translations. The system consists of an $N_{s^{-}}$ site ring of noninteracting spinless fermions in the presence of a uniform magnetic field threading the ring whose total flux is $N_{s} \phi$

$$
H=-t \sum_{\mathbf{j}=1}^{N_{s}}\left(c_{\mathbf{j}}^{\dagger} \exp [i \phi] c_{\mathbf{j}+1}+c_{\mathbf{j}+1}^{\dagger} \exp [-i \phi] c_{\mathbf{j}}\right),
$$

The ground state of this system is non-degenerate and has a non-zero total momentum (non-zero current); i.e., it does not belong to the trivial representation of the group of translations. 
As usual, mathematical concepts have physical relevance whenever there are observable consequences. So, what are the consequences of symmetry breaking? They are:

\section{Generalized rigidity and long-range order}

With the appearance of a broken-symmetry state an order parameter $\psi$ emerges which represents a measure of the degree of asymmetry in the broken-symmetry phase. The general problem of how to explicitly define an order parameter is deferred for a later section, here we will simply assume that we know $\psi$ and it is defined as the expectation value of some space-dependent local observable (order field) $\hat{\psi}(\mathbf{x})$. Since the state is translationally invariant (in the thermodynamic limit) it happens that

$$
\lim _{\left|\mathbf{x}-\mathbf{x}^{\prime}\right| \rightarrow \infty}\left\langle\hat{\psi}(\mathbf{x}) \hat{\psi}\left(\mathbf{x}^{\prime}\right)\right\rangle \rightarrow \psi^{2} .
$$

In other words, the broken symmetry state carries long-range correlations in the order field. Conversely, long-range correlations implies a broken-symmetry state, therefore, long-range correlations is a necessary and sufficient condition for the existence of an ordered state. P.W. Anderson has called this phenomenon generalized rigidity. In his words [8] it is an "emergent property not contained in the simple laws of physics, although it is a consequence of them."

Given a physical Hamiltonian it is not straightforward, in general, to determine whether its ground state is invariant or not. However, under certain conditions (e.g., low space dimensionality) one can certainly establish that there is no broken-symmetry phase and, therefore, no long-range order. Those conditions constitute the hypothesis of the MerminWagner-Hohenberg theorem. The idea behind this theorem is that (thermal or quantum) fluctuations may destroy longrange order, so the conditions of the theorem are directly tied to the reasons that may cause strong fluctuations to the ordered state. Continuous symmetries, low space dimensionality, short-range (constituent) interactions cause stronger fluctuations. Thus at finite temperature, $T>0$, short-range spin models with a continuous symmetry in $d \leq 2$ do not show spontaneous ordering. At $T=0$, the existence of a gap in the excitation spectrum (of a system with a continuous symmetry) precludes long-range order, but the presence of gapless excitations does not necessarily imply this order. (Note that the Mermin-Wagner-Hohenberg and Goldstone (see below) theorems are two complementary aspects of the same fact, and both follow in a unified way from a clever use of the Bogoliubov's inequality [61].)

\section{New massless particles: Nambu-Goldstone modes}

As mentioned above, continuous symmetries play a special role since degeneracies are non-countably infinite. If a continuous symmetry is spontaneously broken, the spectrum of $H$ generally has gapless collective excitations (soft modes). These emergent excitations (quasiparticles), which can be interpreted as new particles with zero mass, are called NambuGoldstone modes or bosons. They are the quantized excitations associated with a spatial twist of the order parameter. The energy of the twist is typically proportional to the inverse of the system's length, i.e., it increases linearly with the wave vector $\mathbf{k}$ of the twist. (The energy of the resulting state van- ishes as $\mathbf{k} \rightarrow \mathbf{0}$.) The number of Nambu-Goldstone bosons is at most $\operatorname{dim} \mathcal{G}-\operatorname{dim} \mathcal{G}_{R}$. The Anderson-Higgs mechanism in gauge theories provides an exception to this theorem, i.e., some (or all, depending upon the symmetry group of the gauge theory) Nambu-Goldstone bosons do not emerge even though the continuous symmetry is broken. The idea behind this mechanism is that after the symmetry is broken, the coupling of our ungauged system to a massless gauge field generates a mass for the gauge field giving rise to massive bosonic excitations.

\section{Topological defects}

The breakdown of long-range order (with temperature, for instance) carries the formation of defect structures such as vortices and domain walls, each characterized by the type of singularity in the order parameter. The topological stability of these defects is defined by the homotopy class of the manifold where the order parameter lives, and work has been developed to mathematically classify these defect structures. The book by P.W. Anderson [8] provides an excellent introduction to the subject.

\section{B. Emergent Symmetry}

In the previous subsection, we revised the fundamental aspects and the deep physical consequences of the concept of broken symmetry. We will introduce now another notion which complements the previous one and also plays a central role in the description of physical systems. This is the notion of emergent symmetry. In broken symmetry phenomenon, the symmetry of the considered system is lowered below a critical temperature. But is it possible to have the opposite situation where the symmetry of our system increases when the temperature is lowered? In a sense that will become clear below, the answer to this question is yes. However, we will see that the emergence of a new symmetry is not signaled by a phase transition but by a crossover between two different energy scales. This new concept is useful to characterize the relevant degrees of freedom that dominate the low-energy physics and the nature of the quasiparticles that result from the interactions between these degrees of freedom.

The isolation of the relevant degrees of freedom for the description of complex systems plays a central role in physics. In general, this process results from a careful separation of the different energy scales involved in the Hamiltonian $H$ under consideration. The elimination of the irrelevant degrees of freedom can be achieved by different methods like perturbation theory, algebraic approaches, or renormalization group. The result is a new low-energy effective Hamiltonian, $H_{\text {eff }}$, that is a valid description of the physical system below some characteristic energy $E_{c}$. The Hilbert space $\mathcal{M}_{0}$ of $H_{\text {eff }}$ is then a subspace of the Hilbert space $\mathcal{H}$ of the original Hamiltonian $H$. Sometimes the spectrum of $H_{\text {eff }}$ and the low-energy spectrum of $H$ are exactly the same. However, in the more general case, the difference between both spectra is finite and can be made arbitrarily small by increasing the distance between the corresponding energy scales in $H$.

We will say that a new symmetry emerges whenever there 
is a symmetry group $G$ of transformations (which is not a group of symmetries of $H$ ) with elements of the form $g_{\alpha}=$ $\sum_{\mathbf{k}} a_{\mathbf{k}} \prod_{\mathbf{j}=1}^{n_{\mathbf{k}}} U_{\mathbf{j}}\left(a_{\mathbf{k}}\right.$ is a $c$-number and $n_{\mathbf{k}}$ an integer), where the unitary operator $U_{\mathbf{j}}$ acts on the local Hilbert space $\mathcal{H}_{\mathbf{j}}$, and

$$
\left[g_{\alpha}, H_{\mathrm{eff}}\right]=0 \quad \text { for any } g_{\alpha} \in G .
$$

The transformations in $G$ are defined in the original Hilbert space $\mathcal{H}$ and leave the subspace $\mathcal{M}_{0}$ invariant. $\mathcal{H}=\bigotimes_{\mathbf{j}} \mathcal{H}_{\mathbf{j}}$ is an arbitrary decomposition of $\mathcal{H}$.] The condition $g_{\alpha}=$ $\sum_{\mathbf{k}} a_{\mathbf{k}} \prod_{\mathbf{j}=1}^{n_{\mathbf{k}}} U_{\mathbf{j}}$ means that the $g_{\alpha}$ 's are linear combinations of $n_{\mathbf{k}}$-local operators (product of $n_{\mathbf{k}}$ operators each of them acting on the local space $\mathcal{H}_{\mathbf{j}}$ ). In this way, we are excluding non-local transformations. This is because for each $g_{\alpha}$ that commutes with $H_{\text {eff }}$, it is always possible to find a non-local operator that commutes with $H$ and is identical to $g_{\alpha}$ when restricted to the subspace $\mathcal{M}_{0}$. Note that if $H_{\text {eff }}$ provides an exact description of the low-energy spectrum of $H$, we say that the emergent symmetry is exact. In contrast, if $H_{\text {eff }}$ is only a very good approximation to the low-energy spectrum of $H$, the emergent symmetry is only an approximate concept. In other words, suppose that $H_{\text {eff }}+H_{\text {eff }}^{\prime}$ is the Hamiltonian that reproduces the exact low-energy spectrum of $H$. If, for instance, we have derived $H_{\text {eff }}$ within perturbation theory to order $n$ in the small parameter $\eta$ (ratio between the small and the large energy scales), $H_{\text {eff }}^{\prime}$ is of order $\eta^{n+m}$, where $m$ is a positive integer. Therefore,

$$
\left[g_{\alpha}, H_{\mathrm{eff}}+H_{\mathrm{eff}}^{\prime}\right]=\left[g_{\alpha}, H_{\mathrm{eff}}^{\prime}\right]=\mathcal{O}\left(\eta^{n+m}\right) \quad \text { for any } \quad g_{\alpha} \in G
$$

Although in these cases the emergent symmetry is an approximate notion, the concept is still useful to identify the nature and properties of the low-energy quasiparticles. This is illustrated in the last examples of this section.

Let us start considering cases in which the notion of emergent symmetry is exact. During last few decades, exact dimer ground states were found for different quantum spin models [10, 62]. The most famous example is the Majumdar-Ghosh solution [9] of the Heisenberg spin-1/2 chain with nearest and second-nearest neighbor interactions $J_{1}$ and $J_{2}$, respectively. The common characteristic of these quantum spin models is the emergence of a local $U(1)$ gauge symmetry that gives rise to the conservation of each local dimer. In general, the dimerized ground states can be written as

$$
\left|\Psi_{0}\right\rangle=\bigotimes_{\left(\mathbf{i}_{1}, \mathbf{i}_{2}\right)}\left|\phi_{s}\left(\mathbf{i}_{1}, \mathbf{i}_{2}\right)\right\rangle
$$

where $\left(\mathbf{i}_{1}, \mathbf{i}_{2}\right)$ denotes a pair of sites and each site belongs to one and only one pair. The wave function $\left|\phi_{s}\left(\mathbf{i}_{1}, \mathbf{i}_{2}\right)\right\rangle$ is the singlet state for the pair $\left(\mathbf{i}_{1}, \mathbf{i}_{2}\right)$. Let us now introduce the following local $U(1)$ transformation

$$
\hat{T}\left(\mathbf{i}_{1}, \mathbf{i}_{2}\right)=\exp \left[i a_{12} P\left(\mathbf{i}_{1}, \mathbf{i}_{2}\right)\right]
$$

where $P\left(\mathbf{i}_{1}, \mathbf{i}_{2}\right)=\left|\phi_{s}\left(\mathbf{i}_{1}, \mathbf{i}_{2}\right)\right\rangle\left\langle\phi_{s}\left(\mathbf{i}_{1}, \mathbf{i}_{2}\right)\right|$ is a local projector on the singlet state of the bond $\left(\mathbf{i}_{1}, \mathbf{i}_{2}\right)$. It is clear that $\hat{T}\left(\mathbf{i}_{1}, \mathbf{i}_{2}\right)\left|\Psi_{0}\right\rangle=\exp \left[i a_{12}\right]\left|\Psi_{0}\right\rangle$. The generators of this $U(1)$ gauge group are the projectors $P\left(\mathbf{i}_{1}, \mathbf{i}_{2}\right)$ which count the number of singlet states on each bond $\left(\mathbf{i}_{1}, \mathbf{i}_{2}\right)$. Then, the set of bonds $\left(\mathbf{i}_{1}, \mathbf{i}_{2}\right)$ provides the natural subsytem decomposition of $\mathcal{H}$. It is important to note that in most of these cases the emergent symmetry is only present in the ground state which is separated from the lowest energy excitations by an energy gap; i.e., $\mathcal{M}_{0}$ is a one-dimensional subspace. Nevertheless, this emergent property of the ground state determines the nature of the low-energy excitations, which are local spin triplets on each bond, that propagate on a sea of singlet dimers. For models like the one of Eq. [88 or Ref. [13], there is an invariant low-energy subspace given by a dimerized ground state and an arbitrary number of triplet excitations, with the condition that triplets cannot be created on dimers which are nearestneighbors. The action of the Hamiltonian restricted to this subspace is invariant under a $U(1)$ gauge transformation and, again, the exact low-energy theory has an infinite number of symmetries not present in the original models.

What is the origin of the emergent $U(1)$ gauge symmetry of quantum dimer ground states of the form of Eq. 151? For most of the cases, if not all, the origin is geometrical frustration. The particular connectivity of the lattice (or lattice topology) produces negative interference between the different links that connect two dimers. When the interference is complete, the dimers become disconnected and the gauge symmetry emerges. This principle is by no means restricted to quantum dimer systems. We can imagine different local structures (instead of dimers) that get disconnected due to the same reason. For instance, currents localized in plaquettes can also result from an emergent $S U(2)$ gauge symmetry in frustrated lattices. As shown in Ref. [63], this occurs for particular fillings of the Hubbard model on a family of frustrated lattices. The guiding principle is always the same. In a certain region of parameters, local currents with two possible orientations become conserved quantities. The local chirality can be described with a pseudospin-1/2 variable that is the relevant degree of freedom to build a low-energy effective theory. Any weak physical interaction that breaks the emergent gauge symmetry can introduce a finite coupling between the local currents and produce different orderings of the chiral degrees of freedom. In the case of Ref. [63], the interaction that breaks the gauge symmetry is an inter-site Coulomb repulsion. This interaction induces an $X Y$-like ordering of the local currents. The low-energy excitations are chiral-waves that are described by magnons in the pseudospin language. Following the same strategy, one can find physical Hamiltonians that give rise to different and, sometimes, unusual low-energy degrees of freedom (see the example of Eq. 157 below).

However frustration is not a requirement to have an exact emergent gauge symmetry. For instance, the Fermi liquid or the band insulator are two examples of emergent symmetries that do not involve any geometrical frustration. These phases of matter are not characterized by any broken symmetry since their corresponding ground states are non-degenerate. According to Landau's theory of Fermi liquids [64], the quasiparticles of the Fermi liquid have an infinite lifetime if they are right at the Fermi surface. This means that the low-energy spectrum of the Fermi liquid is invariant under local $U(1)$ 
transformations of the form $\exp \left[i \phi_{\mathbf{k}_{F}} c_{\mathbf{k}_{F}}^{\dagger} c_{\mathbf{k}_{F}}\right]$ (where $c_{\mathbf{k}_{F}}^{\dagger}$ is the creation operator of a quasiparticle with Fermi wave vector $\mathbf{k}_{F}$ ). The band insulator can also be characterized by a local $U(1)$ emergent symmetry. In this case, the $U(1)$ symmetry can be factorized in real space and is generated by the local transformation: $\exp \left[i a c_{\mathbf{j}}^{\dagger} c_{\mathbf{j}}\right]$. This is the mathematical expression for charge localization that characterizes the insulating state: the charge is locally conserved. Like in the case of the spin dimers, only the ground state of the band insulator exhibits the property of emergent symmetry. In contrast, the dimension of $\mathcal{M}_{0}$ for the Fermi liquid phase is equal to the number of wave vectors $\mathbf{k}_{F}$ that are on the Fermi surface. Note that the Fermi surface is the manifold associated with the group of emergent symmetries whose topology characterizes the universality class of the Fermi liquid.

Exactly and quasi-exactly solvable models provide also examples for exact emergent symmetries. A model is quasiexactly solvable when only part of the spectrum can, in a purely algebraic form, be exactly diagonalized. Let us call $\mathcal{S}_{0}$ the subspace generated by the exactly solvable part of the spectrum. Since $H$ is exactly solvable when restricted to $\mathcal{S}_{0}$, there is a set of operators $g_{\alpha}$ that commute with $H: \mathcal{S}_{0} \rightarrow \mathcal{S}_{0}$. If $\mathcal{S}_{0}$ is also the lowest energy subspace and the operators $g_{\alpha}$ can be factorized as $g_{\alpha}=\sum_{\mathbf{k}} a_{\mathbf{k}} \prod_{\mathbf{j}=1}^{n_{\mathbf{k}}} U_{\mathbf{j}}$, we have another case of emergent symmetry. As an example of a quasi-exactly solvable model that has also an emergent symmetry, we will consider the $t-J_{z}$ chain [6]. The lowest energy subspace of this model can be mapped into the exactly solvable $S=1 / 2$ $X X Z$ chain. This means that $H_{t-J_{z}}: \mathcal{S}_{0} \rightarrow \mathcal{S}_{0}$ has an infinite number of symmetries $g_{n}$ that are linear combinations of $n$-local operators (products of $n$-body spin variables) [65]. These are the quantum symmetries that make the $X X Z$ chain an integrable problem. The relevant low-energy degrees of freedom of $H_{t-J_{z}}$ are holes which are attached to an antiphase domain for the staggered magnetization. For $J_{z}<8 t$, the system is a Luttinger liquid of particles that carry both electrical and topological charges.

We will consider now the cases in which the emergent symmetry is an approximate, albeit important, concept. In the simple example that we describe below, a global $S U(2)$ invariance emerges at low energies. The model is a $d$-dimensional hyper-cubic Kondo lattice with an attractive $(U>0)$ Hubbard interaction for the conduction band, an anisotropic Kondo interaction between the magnetic impurities and the conduction electrons, and a Heisenberg antiferromagnetic interaction $(J>0)$ between the localized spins $(\sigma=\uparrow, \downarrow)$

$$
\begin{aligned}
H^{\mathrm{KA}} & =-t \sum_{\langle\mathbf{i}, \mathbf{j}\rangle, \sigma}\left(c_{\mathbf{i} \sigma}^{\dagger} c_{\mathbf{j} \sigma}+c_{\mathbf{j} \sigma}^{\dagger} c_{\mathbf{i} \sigma}\right)-\mu \sum_{\mathbf{i}} \hat{n}_{\mathbf{i}}-U \sum_{\mathbf{i}} \hat{n}_{\mathbf{i} \uparrow} \hat{n}_{\mathbf{i} \downarrow} \\
& +J_{K} \sum_{\mathbf{i}}\left(\gamma S_{\mathbf{i}}^{z} s_{\mathbf{j}}^{z}+S_{\mathbf{i}}^{x} s_{\mathbf{j}}^{x}+S_{\mathbf{i}}^{y} s_{\mathbf{j}}^{y}\right)+J \sum_{\langle\mathbf{i}, \mathbf{j}\rangle} \mathbf{S}_{\mathbf{i}} \cdot \mathbf{S}_{\mathbf{j}},(153)
\end{aligned}
$$

where $\mathbf{S}_{\mathbf{i}}$ is the spin operator for the localized moment at the site $\mathbf{i}$, and $s_{\mathbf{i}}^{\nu}=1 / 2 \sum_{\tau, \tau^{\prime}} c_{\mathbf{i} \tau}^{\dagger} \sigma_{\tau \tau^{\prime}}^{\nu} c_{\mathbf{i} \tau^{\prime}}$ with $\nu=\{x, y, z\}$. The symmetry group of $H^{\mathrm{KA}}$ is $U(1) \times U(1)$. The corresponding generators or conserved quantities are the total number of particles $\sum_{\mathbf{i}} \hat{n}_{\mathbf{i}}$ and the $z$-component of the total spin $\sum_{\mathbf{i}}\left(S_{\mathbf{i}}^{z}+s_{\mathbf{i}}^{z}\right)$. This model describes the competition between a Kondo-like system $\left(J_{K} \gg U\right)$ and an $s$-wave superconductor coexisting with antiferromagnetic ordering of the localized magnetic moments $\left(J_{K} \ll U\right)$. We will consider here the large $U$ limit $U \gg J_{K}, t$. In this limit, the low-energy subspace $\mathcal{M}_{0}$ is generated by states in which the sites of the conduction band are either empty or double occupied. In other words, the conduction states are non-magnetic and the Kondo interaction is therefore quenched. The low-energy effective model in $\mathcal{M}_{0}$ is

$$
\begin{aligned}
H_{\mathrm{eff}}^{\mathrm{KA}} & =J \sum_{\langle\mathbf{i}, \mathbf{j}\rangle} \mathbf{S}_{\mathbf{i}} \cdot \mathbf{S}_{\mathbf{j}}+\tilde{t} \sum_{\langle\mathbf{i}, \mathbf{j}\rangle, \sigma}\left(\bar{b}_{\mathbf{i}}^{\dagger} \bar{b}_{\mathbf{j}}+\bar{b}_{\mathbf{j}}^{\dagger} \bar{b}_{\mathbf{i}}\right)+2 \tilde{t} \sum_{\langle\mathbf{i}, \mathbf{j}\rangle, \sigma} \bar{n}_{\mathbf{i}} \bar{n}_{\mathbf{j}} \\
& -\tilde{\mu} \sum_{\mathbf{i}} \bar{n}_{\mathbf{i}},
\end{aligned}
$$

where $\tilde{t}=2 t^{2} / U$, and $\tilde{\mu}=2 \mu+\mathrm{z} \tilde{t}$. The hard-core bosons represent the local Cooper pairs

$$
\bar{b}_{\mathbf{i}}^{\dagger}=c_{\mathbf{i} \uparrow}^{\dagger} c_{\mathbf{i} \downarrow}^{\dagger} \quad \bar{b}_{\mathbf{i}}=c_{\mathbf{i} \downarrow} c_{\mathbf{i} \uparrow} .
$$

The first observation is that the localized spins and the conduction electrons are decoupled in $H_{\mathrm{eff}}^{\mathrm{KA}}$. The original Kondo interaction is suppressed by the competing $U$ term. As a consequence, the symmetry group of $H_{\mathrm{eff}}^{\mathrm{KA}}$ is $U(1) \times S U(2)$. The $U(1)$ symmetry is again associated with the conservation of the number of particles that now are Cooper pairs. The emergent spin rotational $S U(2)$ invariance is explicit from the expression $H_{\mathrm{eff}}^{\mathrm{KA}}$ of Eq. (154). This is a simple example of a global $S U(2)$ symmetry that only appears at low energies. The higher-order terms in $J_{K} / U$ will remove this $S U(2)$ invariance leaving the original $U(1)$ symmetry of rotations around the $z$-axis. This means that one of the two Goldstone modes associated to the spontaneously broken $S U(2)$ symmetry will acquire a small mass of order $\left(J_{K} / U\right)^{n}$ with $n \geq 2$.

In the same way an emergent global symmetry is helpful for identifying the nature of the quasiparticles, an emergent gauge symmetry provides a guiding principle for identifying the relevant degrees of freedom at low energies. In general, a noninteracting theory is characterized by local symmetries that express the independence of each particle. For instance, the translation of only one particle is a symmetry for a noninteracting gas. The inclusion of the interactions removes this local symmetry and the many-body problem becomes, in general, non-trivial. However, in many cases it is possible to find another locally gauge-invariant limit for the interacting problem. In this case, the local gauge invariance emerges only at low energies and signals the appearance of effective degrees of freedom that become decoupled. For instance, the origin of antiferromagnetism is more transparent in the strong coupling limit of the half-filled Hubbard model than in the weak or intermediate coupling regimes. Before giving a formal expression for this statement, it is convenient to illustrate its meaning with this simple example. Let us consider a repulsive Hubbard Hamiltonian at half-filling $(U>0)$

$$
\begin{aligned}
H_{\text {Hubb }} & =-t \sum_{\langle\mathbf{i}, \mathbf{j}\rangle, \sigma}\left(c_{\mathbf{i} \sigma}^{\dagger} c_{\mathbf{j} \sigma}+c_{\mathbf{j} \sigma}^{\dagger} c_{\mathbf{i} \sigma}\right)+U \sum_{\mathbf{i}} \hat{n}_{\mathbf{i} \uparrow} \hat{n}_{\mathbf{i} \downarrow} \\
& -\mu \sum_{\mathbf{i}} \hat{n}_{\mathbf{i}}
\end{aligned}
$$


In the infinite- $U$ limit, the low-energy subspace $\mathcal{M}_{0}$ is generated by states having one particle per site, i.e., there is one spin $S=1 / 2$ localized on each site. All states in the manifold $\mathcal{M}_{0}$ have the same energy, and this massive degeneracy is associated with an emergent $S U(2)$ gauge symmetry. In other words, in this limit the local spins get decoupled and are free to rotate without changing the energy of the system. This tells us that the natural degrees of freedom to describe the system in the strong coupling limit are localized spins, instead of itinerant fermions. The presence of an emergent gauge symmetry indicates that there is a limit in our original interacting theory for which some degrees of freedom become noninteracting. The internal structure of these degrees of freedom is determined by the gauge symmetry group. If the system in our example is close to the gauge invariant limit, $U \gg t$, but finite, the relevant degrees of freedom are still the same but they become weakly interacting. This is the origin of antiferromagnetism in the strongly interacting Mott insulators. Since the large- $U$ limit of the Hubbard model is very well known, it may seem to the reader that the use of the new concept of emergent symmetry just provides a complicated way to describe a simple phenomenon. However, this is not the case for the non-trivial examples that are described below. In addition, we will see that the concept of emergent gauge symmetry is important as a guiding principle to find new states of matter that result from strongly interacting systems. One has to keep in mind that even antiferromagnetism remained as a hidden phase for a long period of time.

Let us consider the case of a gauge symmetry group that is a direct product of local symmetry groups, $G=\bigotimes_{\mathrm{k}} G_{\mathrm{k}}$, where each local group $G_{\mathbf{k}}$ acts on the local space $\mathcal{H}_{\mathbf{k}}$. If the reduced Hilbert space $\mathcal{M}_{0}$ admits the decomposition $\mathcal{M}_{0}=\bigotimes_{\mathrm{k}} \mathcal{H}_{\mathrm{k}}$, this will provide the natural basis to write down $H_{\text {eff. }}$. In particular, if $G_{\mathbf{k}}$ is a continuous group, the generators of $G_{\mathbf{k}}$ are conserved quantities at low energies because they commute with $H_{\text {eff. }}$. These generators are physical degrees of freedom and their conservation imply that they are noninteracting. Note that these effective degrees of freedom are the bricks to build new phases out of interacting systems. As soon as we move away (but not too far) from the gauge invariant limit, these degrees of freedom will interact, producing, in some cases, novel types of orderings. The main goal in the rest of this section will be illustrating this phenomenon with different examples.

We will consider now the spin-1/2 ladder of Fig. 10 described by the following Hamiltonian

$$
H^{\mathrm{SL}}=J \sum_{\mathbf{i}, \nu, \nu^{\prime}} \mathbf{S}_{\mathbf{i} \nu} \cdot \mathbf{S}_{\mathbf{i} \nu^{\prime}}+J^{\prime} \sum_{\mathbf{i}, \nu=1,4} \mathbf{S}_{\mathbf{i} \nu} \cdot \mathbf{S}_{\mathbf{i}+1 \nu},
$$

with $J, J^{\prime}>0$ and $1 \leq \nu, \nu^{\prime} \leq 4$. In the limit $J^{\prime} \ll J$, the low-energy subspace of $H^{\mathrm{SL}}$ only contains states in which each square plaquette $\mathbf{i}$ is in a singlet state. This is clear when we analyze the spectrum of an isolated plaquette. The energy of the eigenstates only depends on the total spin $S_{T}: E\left(S_{T}\right)=$ $S_{T}\left(S_{T}+1\right) J$. Thus, the two possible singlets $\left(S_{T}=0\right)$ are the lowest energy states, with the $S_{T}=1$ and $S_{T}=2$ states having eigenvalues $2 J$ and $6 J$, respectively.

We will classify the two local singlet states according to the

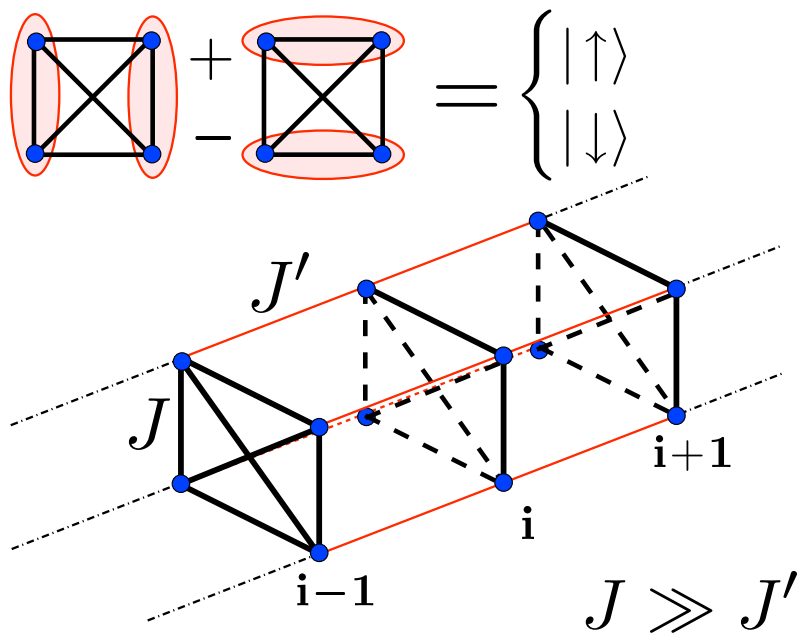

FIG. 10: Four-leg spin ladder. In each plaquette i, there are 4 spin$1 / 2$ which interact through a Heisenberg exchange term of strength $J$. The interaction between plaquettes is $J^{\prime}$, and the links are indicated in the figure.

eigenvalues of the reflection symmetry plane along the diagonal of the plaquette (see Fig. 10). One of the singlet states $\left|S_{\mathbf{i}, s}\right\rangle$ is symmetric under this reflection while the other one, $\left|S_{\mathbf{i}, a}\right\rangle$, is antisymmetric. These two singlet states can be described with an effective pseudospin $\tau=1 / 2$ variable. We will represent the symmetric state $\left|S_{\mathbf{i}, s}\right\rangle=|\uparrow\rangle$ by the eigenvector of $\tau^{z}$ with eigenvalue $1 / 2$ and the antisymmetric one, $\left|S_{\mathrm{i}, a}\right\rangle=|\downarrow\rangle$, by the other eigenvector of $\tau^{z}$. In the infinite$J$ limit, the low-energy degrees of freedom $\tau_{\mathrm{i}}$ are completely decoupled. This situation is similar to the infinite- $U$ limit of the Hubbard model. However, in the present case, the pseudospin variable $\tau_{\mathrm{i}}$ does not represent a magnetic degree of freedom. By means of a canonical transformation to second order in $J^{\prime} / J$, we can derive the following low-energy effective Hamiltonian

$$
H_{\mathrm{eff}}^{\mathrm{SL}}=\frac{J_{\mathrm{eff}}}{4} \sum_{\mathbf{i}}\left(2 \tau_{\mathbf{i}}^{z} \tau_{\mathbf{i}+1}^{z}-\tau_{\mathbf{i}}^{x} \tau_{\mathbf{i}+1}^{x}-\tau_{\mathbf{i}}^{y} \tau_{\mathbf{i}+1}^{y}\right)
$$

where $J_{\text {eff }}=-\left(J^{\prime}\right)^{2} / J . \quad H_{\text {eff }}^{S L}$ is an Ising-like anisotropic Heisenberg model. Since $J_{\text {eff }}<0$, the ground state is a fully polarized ferromagnet that has two possible orientations $\left(Z_{2}\right.$ broken symmetry). In terms of the original variables, the fully polarized states correspond to the direct product of the symmetric and the antisymmetric singlets: $\bigotimes_{\mathrm{i}}\left|S_{\mathbf{i}, s}\right\rangle$ and $\bigotimes_{\mathbf{i}}\left|S_{\mathbf{i}, a}\right\rangle$. The $Z_{2} \times U(1)$ symmetry of $H_{\mathrm{eff}}^{\mathrm{SL}}$ is an emergent symmetry because $H$ is not invariant under the corresponding transformations. These symmetries are explicitely removed when higher order terms in $J^{\prime} / J$ are added to $H_{\text {eff }}^{\mathrm{SL}}$. These terms will stabilize one of the fully polarized ferromagnetic solutions. However, the presence of these terms does not modify the nature of the quasiparticles of $H_{\text {eff }}^{\mathrm{SL}}$. These novel quasiparticles, which we will name kekulons [66], are magnons in the pseudospin language whose spectrum is gapped due to the Ising-like character of $H_{\text {eff }}^{\mathrm{SL}}$. In the original language, these magnons are massive singlet waves. The main effect of the higher-order corrections to $H_{\text {eff }}^{S L}$ is a renormalization of the 
mass (gap) of these quasiparticles. Therefore, even though the emergent symmetry is approximate in the present problem, its most important physical consequences remain valid.

We have seen that there is a systematic procedure for obtaining new types of orderings. The procedure starts from some limit in parameter space, in general the strong coupling limit, in which effective low-energy degrees of freedom are simultaneously stabilized and decoupled. The interactions between these degrees of freedom appear when we move away from this limit and the symmetries or approximate symmetries of these effective interactions have important consequences for the properties of the ground state and the low-energy excitations. These degrees of freedom are the bricks for the novel orderings that result from the effective interactions and simplify the description of the new phase. These novel orderings, that are in general associated with the presence of competing interactions, pose a challenge for experimental physicists to develop probes that are sensitive to the new phases.

\section{BROKEN SYMMETRY PHASE TRANSITIONS}

It is widely recognized that the resolution of interacting systems at a microscopic level requires sophisticated techniques which go beyond the traditional approaches. Much of the current understanding on quantum phases of matter and their corresponding phase transitions is due to renormalization group analysis or the existence of a few exact solutions. The latter, the most desirable scenario, is also the hardest since one seldom encounters solutions in spatial dimensions larger than one. For strongly interacting systems the situation worsens because it is difficult, if not impossible, to identify a small parameter: Their ground states are susceptible to different quantum orderings and low energy excitations because of competing interactions that abound in nonlinearities. Often these systems are near quantum criticality which makes their study even more challenging.

A fundamental notion of universality (or equivalence) naturally emerges from our dictionaries. This notion refers to the fact that many apparently different problems in nature have the same underlying algebraic structure and, therefore, the same physical behavior. In this way, as a result of unveiling the symmetry structure of the problem, there is a concept of physical equivalence hidden in those dictionaries. If the complete Hilbert space of the problem maps onto another in a different language, the concept of universality applies to all length and time scales. On the other hand, if only certain invariant subspaces of the original Hamiltonian map onto another physical system, then, universality will only manifest at certain energy scales.

It was Landau [7] who first noticed the crucial role of symmetry for phase transitions. Since the symmetry of a given state cannot be changed continuously, different symmetries must be associated to states of matter which are qualitatively different. To characterize the symmetry of a given state, Landau introduced the notion of an order parameter. This parameter is zero in the symmetric state and has a nonzero average when the symmetry is broken. Another property of this parameter is that it is non-invariant under at least one of the transformations of the system Hamiltonian. As Landau recognized long time ago, the order parameter is the relevant physical quantity to build a macroscopic theory of thermodynamical phase transitions. However, the search for the order parameter characterizing a broken symmetry state can be a highly non-trivial task.

There are examples in nature of systems entailing hidden microscopic order parameters which do not correspond to macroscopic variables, i.e., they cannot be coupled to any external physical field. To find out these hidden order parameters we cannot avoid a microscopic description of our physical system. It is important to notice the practical consequences of this search: A new microscopic order parameter amounts to predicting a new possible state of matter. The glassy materials are good examples of systems belonging to this class. Since the order parameters characterizing a broken continuous symmetry are associated in general to the generators of the symmetry group, one can imagine that a language based on symmetry generators can shed some light into the search of the possible order parameters for a given system. It is the purpose of this section to illustrate this idea with different examples and to introduce the notion of hierarchical language. One of our goals is to show the fundamental role played by the hierarchical languages in the classification of orders in matter.

\section{A. Classification of Order Parameters}

To illustrate the general procedure of getting and classifying the possible order parameters of a given model, we start discussing the family of Heisenberg models with $S U(N)$ spins in the fundamental representation

$$
H_{\text {Heis }}^{\mathrm{SU}(\mathrm{N})}=J \sum_{\langle\mathbf{i}, \mathbf{j}\rangle} \mathcal{S}^{\mu \nu}(\mathbf{i}) \mathcal{S}^{\nu \mu}(\mathbf{j})
$$

(A summary of the methodology to obtain and classify order parameters is presented at the end of section VIIIB) For $J<0$, the ground states of this family of Hamiltonians can be exactly computed and it is a fully polarized $S U(N)$ ferromagnet. The order parameter is the $S U(N)$ magnetization $\mathcal{S}^{\mu \nu}=\sum_{\mathbf{j}} \mathcal{S}^{\mu \nu}(\mathbf{j})$ which has $N^{2}-1$ (number of generators of $s u(N))$ components. If $J>0$, and the ground state has antiferromagnetic long range order, the order parameter is the staggered $S U(N)$ magnetization $\mathcal{S}_{\mathrm{ST}}^{\mu \nu}=\sum_{\mathbf{j}} e^{i \mathbf{Q} \cdot \mathbf{j}} \mathcal{S}^{\mu \nu}(\mathbf{j})$ ( $Q$ is the antiferromagnetic wave vector). By adding terms which break the $S U(N)$ symmetry explicitly, we can get lower symmetry order parameters associated to the subgroups of $S U(N)$.

We can ask now what is the expression of these order parameters in a spin $S U(2)$ or in a bosonic representation of $H_{\text {Heis }}^{\mathrm{SU}(\mathrm{N})}$. The answer to this question will allow us to explore new phases which can appear in the spin or the bosonic equivalent models. In addition, we will find that the high symmetry points of these models correspond to situations where two or more different phases are coexisting. 
We start with the spin $S U(2)$ representations of $H_{\text {Heis }}^{\mathrm{SU}(\mathrm{N})}$. The local Hilbert space of this $S U(N)$ Heisenberg Hamiltonian has dimension $N$ so we can use an $S U(2)$ spin $S=$ $(N-1) / 2$ to represent this model. In this representation the Hamiltonian Eq. (159) has the following form

$$
H_{\text {Heis }}^{\mathrm{SU}(\mathrm{N})}=J \sum_{\langle\mathbf{i}, \mathbf{j}\rangle} \sum_{l=0}^{N-1} \alpha_{l}\left(\mathbf{S}_{\mathbf{i}} \cdot \mathbf{S}_{\mathbf{j}}\right)^{l},
$$

with the values of $\alpha_{l}$ 's determined from the following system of equations

$$
\begin{aligned}
& \sum_{l=0}^{N-1} \alpha_{l}\left\{[1-S(S+1)]^{l}-(-1)^{l}[S(S+1)]^{l}\right\}=2(-1)^{N}, \\
& \sum_{l=0}^{N-1} \alpha_{l}\left\{\left[\mathcal{Y}_{1}-S(S+1)\right]^{l}-(-1)^{l}[S(S+1)]^{l}\right\}=0 \\
& \sum_{l=0}^{N-1} \alpha_{l}\left\{\left[\mathcal{Y}_{2}-S(S+1)\right]^{l}-[1-S(S+1)]^{l}\right\}=0,
\end{aligned}
$$

where $\mathcal{Y}_{1}=m(2 m+1), \mathcal{Y}_{2}=n(2 n-1)$ and $m, n$ are integers. When $N$ is an odd integer (i.e., $S$ is integer) $m$ and $n$ satisfy $0 \leq m \leq S$, and $1 \leq n \leq S$, while for even $N$ (i.e., $S$ is half-odd integer), $0 \leq m \leq S-1 / 2$, and $1 \leq n \leq$ $S+1 / 2$. It is easy to check that there are $N-1$ linearly independent equations and $N$ variables $\alpha_{l}$. The undetermined variable corresponds to an additive constant.

The simplest case corresponds to $S U(2)$. Here we get the spin $1 / 2(S=(2-1) / 2)$ version

$$
H_{\mathrm{Heis}}^{\mathrm{SU}(2)}=2 J \sum_{\langle\mathbf{i}, \mathbf{j}\rangle} \mathbf{S}_{\mathbf{i}} \cdot \mathbf{S}_{\mathbf{j}}
$$

To understand the general procedure we continue with the first non-trivial example, i.e., $S U(3)$. This particular example has been analyzed in Ref. [46]. By solving the above system of equations we get that the spin one $(S=(3-1) / 2)$ version of the $S U(3)$ Heisenberg Hamiltonian is (up to a constant)

$$
H_{\text {Heis }}^{\mathrm{SU}(3)}=J \sum_{\langle\mathbf{i}, \mathbf{j}\rangle}\left[\mathbf{S}_{\mathbf{i}} \cdot \mathbf{S}_{\mathbf{j}}+\left(\mathbf{S}_{\mathbf{i}} \cdot \mathbf{S}_{\mathbf{j}}\right)^{2}\right]
$$

To fix ideas let us start with the simple ferromagnetic case $J<0$. As mentioned above, the ground state of the ferromagnetic $S U(3)$ Heisenberg model is the state with maximum total $S U(3)$ spin $\mathcal{S}$. The order parameter associated to this broken symmetry is the total $S U(3)$ magnetization $\mathcal{S}^{\mu \nu}=\sum_{\mathbf{j}} \mathcal{S}^{\mu \nu}(\mathbf{j})$ which has eight independent components. We now raise the following question: what is the order parameter of the equivalent model Eq. 163) written in the $(S U(2))$ $S=1$ language? To answer this question we need to write down the relation between the components of $\mathcal{S}^{\mu \nu}$ and the $S=1$ generators. The fundamental theorem guarantees the existence of these mappings. From Eq. (50) and the generalized JW map- pings (section $\mathrm{VC}$, we get (the site index $\mathbf{j}$ is omitted)

$$
\begin{aligned}
S^{x} & =\frac{1}{\sqrt{2}}\left(\mathcal{S}^{01}+\mathcal{S}^{20}+\mathcal{S}^{02}+\mathcal{S}^{10}\right), \\
S^{y} & =\frac{-1}{\sqrt{2} i}\left(\mathcal{S}^{01}+\mathcal{S}^{20}-\mathcal{S}^{02}-\mathcal{S}^{10}\right), \\
S^{z} & =\mathcal{S}^{11}-\mathcal{S}^{22} \\
\left(S^{x}\right)^{2}-\frac{2}{3} & =\frac{1}{2}\left(\mathcal{S}^{12}+\mathcal{S}^{21}+\mathcal{S}^{00}\right),\left(S^{z}\right)^{2}-\frac{2}{3}=-\mathcal{S}^{00}, \\
\left\{S^{x}, S^{y}\right\} & =i\left(\mathcal{S}^{21}-\mathcal{S}^{12}\right), \\
\left\{S^{x}, S^{z}\right\} & =\frac{1}{\sqrt{2}}\left(\mathcal{S}^{01}-\mathcal{S}^{20}-\mathcal{S}^{02}+\mathcal{S}^{10}\right), \\
\left\{S^{y}, S^{z}\right\} & =\frac{-1}{\sqrt{2} i}\left(\mathcal{S}^{01}-\mathcal{S}^{20}+\mathcal{S}^{02}-\mathcal{S}^{10}\right) .
\end{aligned}
$$

The first three operators are the components of the $S=1$ ferromagnetic order parameter, while the second five are the components of the spin-nematic order parameter (components of the bilinear symmetric traceless tensor). The traceless condition implies that $\left(S^{y}\right)^{2}-\frac{2}{3}=-\left(\left(S^{z}\right)^{2}-\frac{2}{3}\right)-\left(\left(S^{x}\right)^{2}-\frac{2}{3}\right)$. In this way, we see that by rotating the $S U(3)$ ferromagnetic order parameter it is possible to evolve from a ferromagnetic to a spin-nematic ground state and vice versa. This means that the $S U(3)$ invariance of the spin Hamiltonian gives rise to coexistence of both phases. Since the algebra $s u(3)$ provides a fundamental representation when the local Hilbert space has $D=3$, any local and linear operator can be written as a linear combination of the identity and the $s u(3)$ generators. The $S U(3)$ magnetization is the highest-dimensional order parameter for the $S=1$ problem. By reducing the symmetry of the Hamiltonian to any subgroup $G$ of $S U(3)$ we can obtain lower symmetry order parameters. The general procedure consists in building a new basis for the generators where each operator transforms according to an irreducible representation of $G$. For instance, if we take $G=S U(2)$ we can do this classification by inverting the Eqs. 164

$$
\begin{aligned}
\mathcal{S}^{00} & =\frac{2}{3}-\left(S^{z}\right)^{2}, \mathcal{S}^{11}=\frac{S^{z}\left(S^{z}+1\right)}{2}-\frac{1}{3}, \\
\mathcal{S}^{10} & =\frac{1}{2 \sqrt{2}}\left[S^{+}+\left\{S^{+}, S^{z}\right\}\right] \\
\mathcal{S}^{01} & =\frac{1}{2 \sqrt{2}}\left[S^{-}+\left\{S^{-}, S^{z}\right\}\right] \\
\mathcal{S}^{20} & =\frac{1}{2 \sqrt{2}}\left[S^{-}-\left\{S^{-}, S^{z}\right\}\right] \\
\mathcal{S}^{02} & =\frac{1}{2 \sqrt{2}}\left[S^{+}-\left\{S^{+}, S^{z}\right\}\right] \\
\mathcal{S}^{12} & =\frac{i}{2}\left\{S^{x}, S^{y}\right\}+\left(S^{x}\right)^{2}+\frac{1}{2}\left(S^{z}\right)^{2}-1, \\
\mathcal{S}^{21} & =\frac{1}{2 i}\left\{S^{x}, S^{y}\right\}+\left(S^{x}\right)^{2}+\frac{1}{2}\left(S^{z}\right)^{2}-1 .
\end{aligned}
$$

In this way we see that the ferromagnetic and the spin-nematic order parameters form a particular basis of $s u(3)$ generators in the fundamental representation. In other words, we can write down any linear operator as a linear combination of the components of both order parameters. Therefore, if a $S=1$ 
Hamiltonian is $S U(2)$ invariant the local order parameter can be either ferromagnetic or spin-nematic since it can be written as a linear combination of both. This exhausts all possible order parameters for an $S U(2)$ invariant $S=1$ Hamiltonian.

Following the previous procedure we can now classify the possible local order parameters of an $S U(2)$ spin $S$ Hamiltonian. The ordinary magnetization is the only phase that can be derived from $S=1 / 2$ spins. It is well-known that a spinnematic phase like the one described above cannot exist for $S=1 / 2$. For $S=1$, we have seen that there is an additional spinnematic phase which appears in a natural way from the connection with the $S U(3)$ group. In general, we can write down a spin $S$ Hamiltonian in terms of the generators of $S U(2 S+1)$ in the fundamental representation. To determine the possible local order parameters of an $S U(N)$ invariant spin $S$ Hamiltonian we have to reduce this space of generators according to the irreducible representations of $S U(2)$. It is easy to check that those representations correspond to the totally symmetric tensors of rank $l \leq 2 S$, i.e., the possible order parameters are generated by application of the $S U(2)$ transformations to the set: $\left\{S^{z},\left(S^{z}\right)^{2}, \cdots,\left(S^{z}\right)^{2 S}\right\} .\left(S^{z}\right)^{l}$ is the highest weight operator of the $S U(2)$ representation associated to the totally symmetric tensor of rank $l$. The dimension of this representation is $2 l+1$. Therefore, the dimension of the space spanned by these operators is $\sum_{l=1}^{2 S}(2 l+1)=(2 S+1)^{2}-1$ which coincides with the dimension of the space of generators of $S U(2 S+1)$. In this way we see that there are $2 S$ independent local order parameters for a spin $S$ problem. The first two correspond to the local magnetization and the local spin-nematic order parameters. To our knowledge, there is no special name for the other multipolar orderings.

This last example illustrates the general procedure to follow in order to determine and classify the possible local order parameters of a given system. It is important to remark that this classification can be made in any representation [46]. For instance, we can re-write the $S U(3)$ Heisenberg Hamiltonian in terms of $S=1 / 2$ hard-core bosons. In that language the local order parameters may be the local magnetization and the Bose-Einstein condensation order parameter $\bar{b}_{\sigma}^{\dagger}$. In this way our algebraic procedure allows one to identify the possible local order parameters of a given system. In addition, the coexistence of more than one order parameter (more than one phase) can be described in a unified way by using an adequate language.

Another connection, which is useful to illustrate the purpose of this section, is the one relating the $S U(N)$ Heisenberg Hamiltonians to $t$ - $J$-like Hamiltonians for hard-core bosons with spin $S=(N-2) / 2$ (i.e., $N_{f}=N-1$ different flavors). To this end we have to use the bosonic expressions for the generators of $S U(N)$ (Eq. 48) introduced in section $\nabla$ Using these expressions we can re-write the $S U(N)$ Heisenberg Hamiltonian in the following way

$$
\begin{aligned}
H_{\mathrm{Heis}}^{\mathrm{SU}(\mathrm{N})} & =\tilde{H}_{\mathrm{Heis}}^{\mathrm{SU}(\mathrm{N}-1)}+J \sum_{\langle\mathbf{i}, \mathbf{j}\rangle, \alpha}\left[\bar{b}_{\mathbf{i} \alpha}^{\dagger} \bar{b}_{\mathbf{j} \alpha}+\bar{b}_{\mathbf{j} \alpha}^{\dagger} \bar{b}_{\mathbf{i} \alpha}\right] \\
& +J \sum_{\langle\mathbf{i}, \mathbf{j}\rangle} \bar{n}_{\mathbf{i}} \bar{n}_{\mathbf{j}}-\mathrm{z} J \frac{N-1}{N} \sum_{\mathbf{j}} \bar{n}_{\mathbf{j}}
\end{aligned}
$$

where $\bar{n}_{\mathbf{j}}=\sum_{\alpha=1}^{N_{f}} \bar{n}_{\mathbf{j} \alpha}$, and $\tilde{H}_{\text {Heis }}^{\mathrm{SU}(\mathrm{N}-1)}$ is given by

$$
\tilde{H}_{\text {Heis }}^{\mathrm{SU}(\mathrm{N}-1)}=J \sum_{\langle\mathbf{i}, \mathbf{j}\rangle,(\mu, \nu)=1, N_{f}} \mathcal{S}^{\mu \nu}(\mathbf{i}) \mathcal{S}^{\nu \mu}(\mathbf{j}) .
$$

This mapping is valid for any spatial dimension $d$. If we write down the same Hamiltonian using fermions instead of hardcore bosons, a gauge field appears in $d=2$ due to the presence of the $K_{\mathbf{j}}$ operators which transmute the statistics. When $d=1$ the Hamiltonian is exactly the same for fermions and hard-core bosons (and anyons, in general). In this particular language we can check that the ferromagnetic $S U(N)$ order parameter describes the coexistence of all the magnetic phases associated to the $S U(N-1)$ Heisenberg Hamiltonian (for instance, ferromagnetic and spin-nematic phases for $N=4$ ), a Bose-Einstein condensate for each of the $N-1$ different flavors, and a homogeneous $(\mathbf{k}=0)$ charge density wave.

Let us start analyzing the simplest $N=2$ case (Eq. (162). This case corresponds to spinless bosons with a kinetic energy term and a nearest-neighbor density-density interaction. There are no spin degrees of freedom for this particular case and the $S U(2)$ invariance is then associated to the charge degrees of freedom. The two phases which coexist in this case are the Bose-Einstein condensate and the uniform charge density wave order. For $N=3$ we have the usual $t$ - $J$ Hamiltonian for spin $S=1 / 2$ hard-core bosons (or fermions in $1 d$ ). In this case the $S U(3)$ symmetry gives rise to a coexistence between $S=1 / 2$ ferromagnetism, Bose-Einstein condensation in both flavors, and the uniform charge density wave. These orders are different components of the same $S U(3)$ order parameter (see Eq. 50). In this particular language, $\bar{b}_{\uparrow}^{\dagger}=\mathcal{S}^{10}$, $\bar{b}_{\downarrow}^{\dagger}=\mathcal{S}^{20}, \bar{b}_{\uparrow}=\mathcal{S}^{01}$, and $\bar{b}_{\downarrow}=\mathcal{S}^{02}$ are the components of the order parameter for the spin up and down Bose-Einstein condensates. For the magnetization we get $s^{z}=\frac{1}{2}\left(\mathcal{S}^{11}-\mathcal{S}^{22}\right)$, $s^{x}=\frac{1}{2}\left(\mathcal{S}^{12}+\mathcal{S}^{21}\right)$, and $s^{y}=\frac{1}{2 i}\left(\mathcal{S}^{12}-\mathcal{S}^{21}\right)$. The fact that $\bar{n}_{\mathbf{j}}=\frac{2}{3}-\mathcal{S}^{00}$ can take any value by making $S U(3)$ rotations of the ground states is another manifestation of the BoseEinstein condensation. In addition, $\bar{n}_{\mathbf{j}}$ is the order parameter associated to the uniform charge density wave (if the $S U(3)$ magnetization is oriented along the charge density wave direction we obtain $\bar{n}_{\mathbf{j}}=1$ in each site). For $N=4$ we get a model which describes a gas of $S=1$ hard-core bosons with the bilinear-biquadratic magnetic interaction of Eq. (163). In this case we get coexistence of ferromagnetism, spin-nematic, Bose-Einstein condensation for the three different flavors, and a uniform charge density wave. As in the previous case there is an additional phase when $N$ is increased by one.

Therefore, this family of $S U(N)$ Heisenberg Hamiltonians naturally describes a multi-phase behavior when the original language, based upon the generators of $S U(N)$, is translated into another based on generators of a lower symmetry group. This illustrates a general rule which applies to any pair of groups $(G, \mathcal{G})$ such that $G$ is a subgroup of $\mathcal{G}$, and the order parameter $\mathbf{P}$ transforms according to an irreducible representation of $\mathcal{G}$. In general, this representation will be reducible under the operations of the lower symmetry group $G$, i.e., we will be able to express the given representation as a direct sum of representations which are irreducible under the application 
of $G$. We can associate an order parameter $\mathbf{p}_{\gamma}$ to each of these representations. If we add the dimensions of each of these $\mathbf{p}_{\gamma}$ order parameters, we will get the dimension of $\mathbf{P}$. Therefore, by using the lower symmetry language, we obtain different phases, and each of them is characterized by one order parameter $\mathbf{p}_{\gamma}$.

In the previous analysis we have not discussed the possible spatial dependence of the order parameter. In other words, we assumed that it was homogeneous over the entire lattice. However, there are many instances in nature where the order parameter is non-uniform. The antiferromagnetism is one of the most common examples. The previous analysis can be easily extended to the case of non-uniform order parameters with a well-defined wavevector $\mathbf{k}$. We just need to recognize that the non-uniform order parameters can be written as a Fourier transform of the local order parameter $\mathbf{p}(\mathbf{j})$ if the model considered has lattice translation invariance. Indeed, the presence of a non-uniform order parameter indicates that the translation symmetry has been spontaneously broken. Therefore the expression for the non-uniform order parameter $\hat{\mathbf{p}}(\mathbf{k})$ is

$$
\hat{\mathbf{p}}(\mathbf{k})=\frac{1}{N_{s}} \sum_{\mathbf{j}} \exp [i \mathbf{k} \cdot \mathbf{j}] \mathbf{p}(\mathbf{j})
$$

Since the previous analysis is applied to the local order parameters $\mathbf{p}(\mathbf{j})$, Eq. 168 shows that conclusions are trivially extended to non-uniform global order parameters.

\section{B. Hierarchical Languages: The Quantum Phase Diagram of the Bilinear-Biquadratic Heisenberg Model}

We have seen that the local order parameter acquires its simplest form when it is expressed in terms of the hierarchical language. In addition, the generators of this language exhaust all possible local order parameters which may result from the solution of the problem under consideration. In other words, any local order parameter can be written as a linear combination of generators of the hierarchical language. The Hamiltonians considered above are special cases since they have an $S U(N)$ invariance and therefore correspond to high symmetry points of an eventual phase diagram. We just considered those cases as the simplest examples of coexistence of different phases and unification of order parameters. We will show now that high symmetry is not a requirement for the successful application of the present formalism to the determination of quantum phase diagrams. To this end we will consider the most general isotropic $S U(2) S=1$ model with nearestneighbor interactions in a hypercubic lattice (an overal $J>0$ factor is omitted in the following)

$$
H_{\phi}=\sqrt{2} \sum_{\langle\mathbf{i}, \mathbf{j}\rangle}\left[\cos \phi \mathbf{S}_{\mathbf{i}} \cdot \mathbf{S}_{\mathbf{j}}+\sin \phi\left(\mathbf{S}_{\mathbf{i}} \cdot \mathbf{S}_{\mathbf{j}}\right)^{2}\right],
$$

a model already introduced in Eq. 83, . The parameter $\phi$ sets the relative strength between the bilinear and biquadratic terms. Eq. 163 corresponds to the particular case $\phi=5 \pi / 4$. Indeed, as it is shown below, there are four isolated values of $\phi=\{\pi / 4, \pi / 2,5 \pi / 4,3 \pi / 2\}$ for which $H_{\phi}$ is $S U(3)$ invariant. The only symmetry which is present for any value of $\phi$ is the global $S U(2)$ invariance since $H_{\phi}$ is a function of the scalar products $\mathbf{S}_{\mathbf{i}} \cdot \mathbf{S}_{\mathbf{j}}$.

The Hamiltonian $H_{\phi}$ has been the subject of several studies in the last two decades [14, 67, 68, 69, 70, 71, 72, 73, 74, 75, 76, 77, 78], nevertheless, the complete characterization of the different phases was not, until now, completely solved. A semiclassical treatment for $d>1[14]$ indicates that there are four different phases: the usual ferromagnetic $(\pi / 2<\phi<$ $5 \pi / 4)$ and antiferromagnetic $(3 \pi / 2<\phi<\pi / 4)$ phases are separated on both sides by collinear- $(5 \pi / 4<\phi<3 \pi / 2)$ and orthogonal-nematic $(\pi / 4<\phi<\pi / 2)$ orderings. We will show below that the collinear- and orthogonal-nematic phases obtained with the semiclassical approximation are replaced by uniform- and staggered-nematic orderings, respectively.

As we have seen in the previous subsection, the $S U(3)$ spins in the fundamental representation and the $S=1 S U(2)$ spins are two equivalent languages. In addition, we have shown in the sections $\mathrm{VA}$ and $\mathrm{VC}$ that the $S U(3)$ spins and the $S=1 S U(2)$ spins can be respectively mapped onto $S=1 / 2$ hard-core bosons. We will use now these transformations to map the spin one Hamiltonian $H_{\phi}$ onto its $S U(3)$ spin version. For pedagogical reasons, it is convenient to use the $S=1 / 2$ hard-core bosons as an intermediate language.

In section $\mathrm{VA}$ we introduced a spin-particle transformation connecting $S U(N)$ spins and multiflavored hard-core bosons (JW particles, in general). In particular, the fundamental (quark) representations of $S U(N)$ were mapped onto an algebra of hard-core bosons with $N_{f}=N-1$ flavors (see Eq. (48)). For $N=3$ the hard-core bosons have two flavors ( $\alpha=\uparrow, \downarrow$ ) which can be associated to an internal spin $S=1 / 2$ degree of freedom. A compact way of writing the $S U(3)$ spin in terms of hard-core bosons is

$$
\mathcal{S}(\mathbf{j})=\left(\begin{array}{ccc}
\frac{2}{3}-\bar{n}_{\mathbf{j}} & \bar{b}_{\mathbf{j} \uparrow} & \bar{b}_{\mathbf{j} \downarrow} \\
\bar{b}_{\mathbf{j} \uparrow}^{\dagger} & \bar{n}_{\mathbf{j} \uparrow}-\frac{1}{3} & \bar{b}_{\mathbf{j} \uparrow}^{\dagger} \bar{b}_{\mathbf{j} \downarrow} \\
\bar{b}_{\mathbf{j} \downarrow}^{\dagger} & \bar{b}_{\mathbf{j} \downarrow}^{\dagger} \bar{b}_{\mathbf{j} \uparrow} & \bar{n}_{\mathbf{j} \downarrow}-\frac{1}{3}
\end{array}\right) .
$$

It is straightforward to write down each generator of the $s u(3)$ algebra in terms of the Gell-Mann (traceless Hermitian) matrices

$$
\begin{aligned}
\lambda_{1}= & \left(\begin{array}{lll}
0 & 1 & 0 \\
1 & 0 & 0 \\
0 & 0 & 0
\end{array}\right), \lambda_{2}=\left(\begin{array}{ccc}
0 & -i & 0 \\
i & 0 & 0 \\
0 & 0 & 0
\end{array}\right), \lambda_{3}=\left(\begin{array}{ccc}
1 & 0 & 0 \\
0 & -1 & 0 \\
0 & 0 & 0
\end{array}\right), \\
\lambda_{4}= & \left(\begin{array}{lll}
0 & 0 & 1 \\
0 & 0 & 0 \\
1 & 0 & 0
\end{array}\right), \lambda_{5}=\left(\begin{array}{ccc}
0 & 0 & -i \\
0 & 0 & 0 \\
i & 0 & 0
\end{array}\right), \lambda_{6}=\left(\begin{array}{lll}
0 & 0 & 0 \\
0 & 0 & 1 \\
0 & 1 & 0
\end{array}\right), \\
& \lambda_{7}=\left(\begin{array}{ccc}
0 & 0 & 0 \\
0 & 0 & -i \\
0 & i & 0
\end{array}\right), \lambda_{8}=\frac{1}{\sqrt{3}}\left(\begin{array}{ccc}
1 & 0 & 0 \\
0 & 1 & 0 \\
0 & 0 & -2
\end{array}\right),
\end{aligned}
$$


with the result

$$
\begin{aligned}
\bar{b}_{\mathbf{j} \uparrow}^{\dagger} & =\frac{\lambda_{1}+i \lambda_{2}}{2}, \bar{b}_{\mathbf{j} \uparrow}=\frac{\lambda_{1}-i \lambda_{2}}{2}, \\
\bar{b}_{\mathbf{j} \downarrow}^{\dagger} & =\frac{\lambda_{6}-i \lambda_{7}}{2}, \bar{b}_{\mathbf{j} \downarrow}=\frac{\lambda_{6}+i \lambda_{7}}{2}, \\
\bar{b}_{\mathbf{j} \uparrow}^{\dagger} \bar{b}_{\mathbf{j} \downarrow} & =\frac{\lambda_{4}+i \lambda_{5}}{2}, \bar{b}_{\mathbf{j} \downarrow}^{\dagger} \bar{b}_{\mathbf{j} \uparrow}=\frac{\lambda_{4}-i \lambda_{5}}{2}, \\
\bar{n}_{\mathbf{j} \uparrow}-\frac{1}{3} & =\frac{\lambda_{3}}{2}+\frac{\lambda_{8}}{2 \sqrt{3}}, \bar{n}_{\mathbf{j} \downarrow}-\frac{1}{3}=-\frac{\lambda_{8}}{\sqrt{3}} .
\end{aligned}
$$

This constitutes the Cartan-Weyl representation of $s u(3)$ and illustrates the fact that a $S=1 S U(2)$ spin can be equally represented in terms of quark fields.

In the same way we wrote in Eq. 170 the generators of $S U(3)$ in the fundamental representation, we can write down the corresponding expressions for the generators in the conjugate representation

$$
\tilde{\mathcal{S}}(\mathbf{j})=\left(\begin{array}{ccc}
\frac{2}{3}-\bar{n}_{\mathbf{j}} & -\bar{b}_{\mathbf{j} \downarrow}^{\dagger} & -\bar{b}_{\mathbf{j} \uparrow}^{\dagger} \\
-\bar{b}_{\mathbf{j} \downarrow} & \bar{n}_{\mathbf{j} \downarrow}-\frac{1}{3} & \bar{b}_{\mathbf{j} \uparrow}^{\dagger} \bar{b}_{\mathbf{j} \downarrow} \\
-\bar{b}_{\mathbf{j} \uparrow} & \bar{b}_{\mathbf{j} \downarrow}^{\dagger} \bar{b}_{\mathbf{j} \uparrow} & \bar{n}_{\mathbf{j} \uparrow}-\frac{1}{3}
\end{array}\right) .
$$

When the $S=1$ operators are replaced by the corresponding functions of $S U(3)$ generators in the fundamental and the conjugate representations, it turns out that $H_{\phi}$, up to an irrelevant constant, is a linear combination of the ferromagnetic and the antiferromagnetic $S U(3)$ Heisenberg models

$$
\begin{aligned}
H_{\phi} & =\sqrt{2} \sum_{\langle\mathbf{i}, \mathbf{j}\rangle}\left[\cos \phi \mathcal{S}^{\mu \nu}(\mathbf{i}) \mathcal{S}^{\nu \mu}(\mathbf{j})\right. \\
& \left.+(\sin \phi-\cos \phi) \mathcal{S}^{\mu \nu}(\mathbf{i}) \tilde{\mathcal{S}}^{\nu \mu}(\mathbf{j})\right] .
\end{aligned}
$$

Repeated greek superindices are summed and the site index $\mathbf{i}$ runs over one of the two sublattices. This expression for $H_{\phi}$ illustrates the very important result that any nonlinear interaction in the original representation is simply a bilinear term in the new representation when mapped onto the highest rank algebra [46]. In particular, as mentioned above, there are certain special points in parameter space where the Hamiltonian is highly symmetric. For example, for $\phi=\frac{\pi}{4}$ and $\frac{5 \pi}{4}, H_{\phi}$ is explicitly invariant under uniform $S U(3)$ transformations on the spins [48], while for $\phi=\frac{\pi}{2}, \frac{3 \pi}{2}, H_{\phi}$ is explicitly invariant under staggered conjugate rotations of the two sublattices. These symmetries are hard to identify in the original spin representation but are manifest in the $S U(3)$ representation.

In the following we will concentrate on the determination of the quantum phase diagram of $H_{\phi}$ for spatial dimensions $d>$ 1 to avoid the strong effects of quantum fluctuations which can restore the continuous symmetry when $d=1$. In the previous section we have analyzed the high symmetry point $\phi=\frac{5 \pi}{4}$. We found that the ground state has a non-zero order parameter

$$
\mathcal{S}=\sum_{\mathbf{j}} \mathcal{S}(\mathbf{j}),
$$

associated to a broken continuous $S U(3)$ symmetry. This order parameter is the uniform $S U(3)$ magnetization and corresponds to the coexistence of a ferromagnetic and a uniform spin-nematic ordering (see Eq. (164). This indicates that $\phi=\frac{5 \pi}{4}$ is a quantum phase transition point separating a ferromagnetic phase from a uniform spin-nematic one. Let us consider now the related point $\phi=\frac{\pi}{4}$ which differs in an overall sign from the previous case. This sign changes the interaction from ferromagnetic to antiferromagnetic. Therefore, for this new high symmetry point we expect to get a ground state characterized by the staggered order parameter

$$
\mathcal{S}_{\mathrm{ST}}=\sum_{\mathbf{j}} \exp [i \mathbf{Q} \cdot \mathbf{j}] \mathcal{S}(\mathbf{j})
$$

where $\mathbf{Q}$ is the antiferromagnetic wave vector. It is clear from Eq. (164), that this staggered $S U(3)$ order parameter corresponds to the coexistence of the staggered $S U(2)$ magnetization

$$
\mathbf{M}_{\mathrm{ST}}=\sum_{\mathbf{j}} \exp [i \mathbf{Q} \cdot \mathbf{j}] \mathbf{S}_{\mathbf{j}}
$$

and the staggered nematic order parameter

$$
\mathbf{N}_{\mathrm{ST}}=\sum_{\mathbf{j}} \exp [i \mathbf{Q} \cdot \mathbf{j}] \mathbf{N}_{\mathbf{j}}
$$

$\mathbf{N}_{\mathbf{j}}$ is the symmetric and traceless component of the tensor obtained from the tensorial product of two vectors $\mathbf{S}_{\mathbf{j}}$. Hence, $\phi=\frac{\pi}{4}$ is a transition point separating the usual antiferromagnetic ordering from a staggered spin-nematic phase characterized by the order parameter of Eq. 176.

We will consider now the other two high-symmetry points, $\phi=\frac{\pi}{2}, \frac{3 \pi}{2}$. For $\phi=\frac{3 \pi}{2}$, the $S U(3)$ symmetry is generated by the staggered operator

$$
\mathcal{S}_{+}=\sum_{\mathbf{j} \in A} \mathcal{S}(\mathbf{j})+\sum_{\mathbf{j} \in B} \tilde{\mathcal{S}}(\mathbf{j}),
$$

where $A$ and $B$ denote the two different sublattices of a hypercubic lattice. In this case, we have a ferromagnetic interaction between $\mathcal{S}(\mathbf{i})$ and $\tilde{\mathcal{S}}(\mathbf{j})$, and then $\mathcal{S}_{+}$is the order parameter characterizing the broken $S U(3)$ symmetry of the ground state. It is interesting to note that when the $S U(3)$ order parameter $\mathcal{S}_{+}$is reduced with respect to the $S U(2)$ group, the two coexisting order parameters are the staggered magnetization (see Eq. 175) and the uniform nematic order parameter

$$
\mathbf{N}=\sum_{\mathbf{j}} \mathbf{N}_{\mathbf{j}}
$$

In other words, if we apply an $S U(3)$ rotation generated by $\mathcal{S}_{+}$to the staggered magnetization we get the uniform nematic order parameter, and vice versa, the uniform nematic order parameter is rotated into the staggered magnetization. This can be immediately seen by writing down the components of the local $S U(2)$ magnetization and the nematic order parameter as a function of the local generators of $s u(3)$ in the conjugate 
representation $\tilde{\mathcal{S}}(\mathbf{j})$

$$
\begin{aligned}
S^{x} & =\frac{-1}{\sqrt{2}}\left(\tilde{\mathcal{S}}^{01}+\tilde{\mathcal{S}}^{20}+\tilde{\mathcal{S}}^{02}+\tilde{\mathcal{S}}^{10}\right) \\
S^{y} & =\frac{1}{\sqrt{2} i}\left(\tilde{\mathcal{S}}^{01}+\tilde{\mathcal{S}}^{20}-\tilde{\mathcal{S}}^{02}-\tilde{\mathcal{S}}^{10}\right), \\
S^{z} & =\tilde{\mathcal{S}}^{22}-\tilde{\mathcal{S}}^{11}, \\
\left(S^{x}\right)^{2}-\frac{2}{3} & =\frac{1}{2}\left(\tilde{\mathcal{S}}^{12}+\tilde{\mathcal{S}}^{21}+\tilde{\mathcal{S}}^{00}\right),\left(S^{z}\right)^{2}-\frac{2}{3}=-\tilde{\mathcal{S}}^{00}, \\
\left\{S^{x}, S^{y}\right\} & =i\left(\tilde{\mathcal{S}}^{21}-\tilde{\mathcal{S}}^{12}\right), \\
\left\{S^{x}, S^{z}\right\} & =\frac{1}{\sqrt{2}}\left(\tilde{\mathcal{S}}^{01}-\tilde{\mathcal{S}}^{20}-\tilde{\mathcal{S}}^{02}+\tilde{\mathcal{S}}^{10}\right), \\
\left\{S^{y}, S^{z}\right\} & =\frac{-1}{\sqrt{2} i}\left(\tilde{\mathcal{S}}^{01}-\tilde{\mathcal{S}}^{20}+\tilde{\mathcal{S}}^{02}-\tilde{\mathcal{S}}^{10}\right) .
\end{aligned}
$$

Comparing these expressions to the ones in Eq. 164, we see that when we change from $\mathcal{S}(\mathbf{j})$ to $\tilde{\mathcal{S}}(\mathbf{j})$, there is a change in sign for the three components associated to the magnetization, while the five components corresponding to the nematic parameter remain the same. Then, it is clear that $\mathcal{S}_{+}$describes the coexistence of a staggered magnetization and a uniform nematic ordering. Therefore, the conclusion is that $\phi=\frac{3 \pi}{2}$ separates an ordinary antiferromagnetic phase from the uniform nematic ordering.

The last high symmetry point to be considered is $\phi=\frac{\pi}{2}$. In this case the coupling between $\mathcal{S}(\mathbf{i})$ and $\tilde{\mathcal{S}}(\mathbf{j})$ turns out to be positive, i.e., antiferromagnetic, and therefore we expect to get a broken continuous symmetry characterized by the order parameter

$$
\mathcal{S}_{-}=\sum_{\mathbf{j} \in A} \mathcal{S}(\mathbf{j})-\sum_{\mathbf{j} \in B} \tilde{\mathcal{S}}(\mathbf{j})
$$

From the considerations above, it is clear that $\mathcal{S}_{-}$describes the coexistence of ferromagnetism (uniform magnetization) and staggered nematic order. Hence, $\phi=\frac{\pi}{2}$ is a transition point separating these two phases.

\begin{tabular}{|c||c|c|c|}
\hline$\phi$ & Global $S U(3)$ OP & OP 1 & OP 2 \\
\hline \hline $5 \pi / 4$ (FM-UN) & $\mathcal{S}=\sum_{\mathbf{j}} \mathcal{S}(\mathbf{j})$ & $\mathbf{M}$ & $\mathbf{N}$ \\
\hline$\pi / 4$ (AF-SN) & $\mathcal{S}_{\mathrm{ST}}=\sum_{\mathbf{j}} \exp [i \mathbf{Q} \cdot \mathbf{j}] \mathcal{S}(\mathbf{j})$ & $\mathbf{M}_{\mathrm{ST}}$ & $\mathbf{N}_{\mathrm{ST}}$ \\
\hline $3 \pi / 2(\mathrm{AF}-\mathrm{UN})$ & $\mathcal{S}_{+}=\sum_{\mathbf{j} \in A} \mathcal{S}(\mathbf{j})+\sum_{\mathbf{j} \in B} \tilde{\mathcal{S}}(\mathbf{j})$ & $\mathbf{M}_{\mathrm{ST}}$ & $\mathbf{N}$ \\
\hline$\pi / 2(\mathrm{FM}-\mathrm{SN})$ & $\mathcal{S}_{-}=\sum_{\mathbf{j} \in A} \mathcal{S}(\mathbf{j})-\sum_{\mathbf{j} \in B} \tilde{\mathcal{S}}(\mathbf{j})$ & $\mathbf{M}$ & $\mathbf{N}_{\mathrm{ST}}$ \\
\hline
\end{tabular}

TABLE III: Order parameters describing the different phases of the bilinear-biquadratic $S=1$ Heisenberg model for $d>1$. $\phi$ indicates the phase boundary where the two phases in parentheses coexist.

In this way, by identifying the high-symmetry points of $H_{\phi}$ we have determined the quantum phase diagram of this model (see Fig. 11). In addition to the transition points, we have obtained explicit expressions for the order parameters associated to each phase for any $d>1$; these are summarized in table III We can also predict from this analysis that the four transition points (high-symmetry points) correspond to first order quantum phase transitions. In each phase, the corresponding order parameter has a a finite value and they coexist pairwise at the high-symmetry points. However, as soon as we depart from this point in one or the other direction in $\phi$, the $S U(3)$ symmetry is removed and one of the order parameters goes discontinuously to zero. In other words, the states with pure magnetic (ferro or antiferromagnetic) and nematic orderings belong to different representations of $S U(2)$ (the remaining symmetry) so only one of them remains as the ground state when the $S U(3)$ symmetry is lifted.

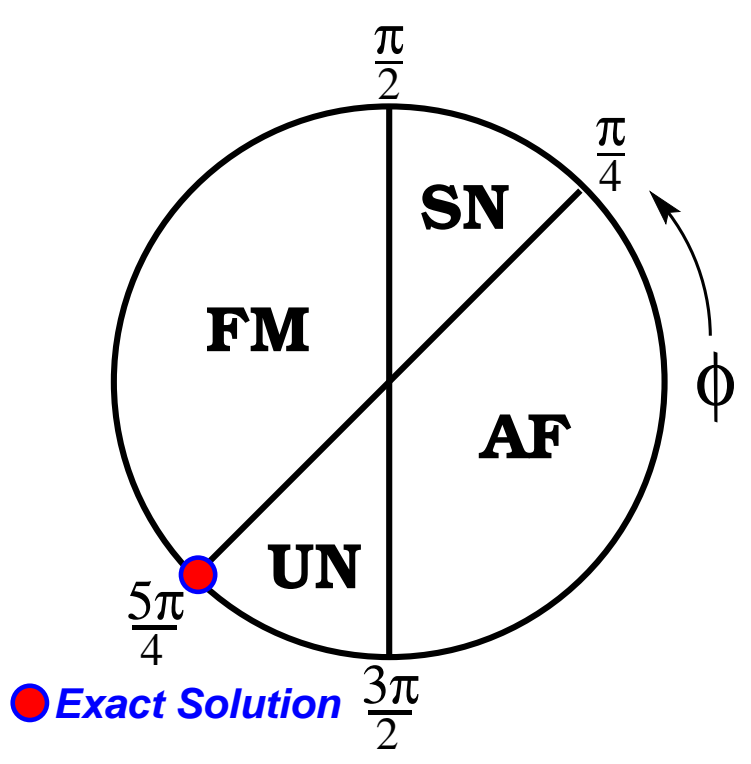

FIG. 11: Quantum phase diagram of the bilinear-biquadratic $S U(2)$ $S=1$ model for $d>1$. The phases are: AF: antiferromagnetic; $\mathrm{SN}$ : staggered-nematic, FM: ferromagnetic, and UN: uniform-nematic.

In closing this section let us summarize the main steps to follow in order to obtain and classify the local order parameters.

- Identify the group $\mathcal{G}_{\mathrm{HL}}=S U(D)$ associated to the hierarchical language whose fundamental representation has the same dimension $D$ as the local Hilbert space of the problem. The generators of this language exhaust all possible local order parameters.

- Identify the group of global symmetries of the Hamiltonian $\mathcal{G}$ which are direct products of local transformations.

- Given that $\mathcal{G} \subseteq \mathcal{G}_{\mathrm{HL}}$, then one can classify the generators of $\mathcal{G}_{\mathrm{HL}}$ in the fundamental representation according to the irreps of $\mathcal{G}$. Each irrep leads to a different broken symmetry order parameter.

- Key: existence of a general set of $S U(D)$ transformations. 


\section{LATTICE GAUGE THEORIES AND THE QUANTUM LINK MODELS}

Wilson's lattice formulation of quantum field theory provides a non-perturbative regularization to Euclidean functional integrals [79]. The space-time lattice supplies the field theory with a cutoff such that loop integrations in perturbation theory yields finite results instead of divergences.

In this way, a natural analogy emerges between quantum field theory on a $d$-dimensional lattice and classical statistical mechanics in $d+1$, which for a pure gauge theory (i.e., gauge theories without matter fields) amounts to consider the classical lattice action:

$$
S[u]=-\sum_{\mathbf{i}, \mu \neq \nu} \operatorname{Tr}\left\{u_{\mathbf{i}, \mu} u_{\mathbf{i}+\hat{\mu}, \nu} u_{\mathbf{i}+\hat{\nu}, \mu}^{\dagger} u_{\mathbf{i}, \nu}^{\dagger}\right\} .
$$

The classical complex parallel transporters $u_{\mathbf{i}, \mu}$ transform as:

$$
u_{\mathbf{i}, \mu}^{\prime}=\Lambda^{-1}(\mathbf{i}) u_{\mathbf{i}, \mu} \Lambda(\mathbf{i}+\hat{\mu})
$$

under local gauge transformations $\Lambda(\mathbf{i})$ which leave the action $S[u]$ invariant. The elementary parallel transporters are associated with an ordered pair of points in the lattice (links). For instance, the link $(\mathbf{i}, \mu)$ corresponds to the straight path from lattice site $\mathbf{i}$ to $\mathbf{i}+\hat{\mu}$.

Recently, Wiese and co-workers [80] have further elaborated work started by Horn in 1981 [81] on a new way of nonperturbative regularization of field theories which they named D-theory. In D-theory the Euclidean action in the standard Wilsonian formulation of a $d$-dimensional lattice field theory is replaced by a Hamilton operator $H$

$$
H=\sum_{\mathbf{i}, \mu \neq \nu} \operatorname{Tr}\left\{U_{\mathbf{i}, \mu} U_{\mathbf{i}+\hat{\mu}, \nu} U_{\mathbf{i}+\hat{\nu}, \mu}^{\dagger} U_{\mathbf{i}, \nu}^{\dagger}\right\}
$$

with quantum link operators $U_{\mathbf{i}, \mu}$ on a $d$-dimensional lattice, which constitute generators of an algebra acting on a Hilbert space (e.g, a $U(1)$ quantum link model can be realized with quantum links that satisfy an $s u(2)$ algebra). $H$ commutes with the local generators of gauge transformations $\mathbf{G}_{\mathbf{i}}\left(U_{\mathbf{i}, \mu}^{\prime}=\prod_{\mathbf{j}} \Lambda^{-1}(\mathbf{j}) U_{\mathbf{i}, \mu} \prod_{\mathbf{l}} \Lambda(\mathbf{l})\right.$ with $\left.\Lambda(\mathbf{i})=e^{i \alpha_{\mathbf{i}} \cdot \mathbf{G}_{\mathbf{i}}}\right)$ and the theory is defined through the quantum partition function $\mathcal{Z}=\operatorname{Tr}\left\{e^{-\beta H}\right\}$, where the trace is taken on the Hilbert space.

As emphasized in Ref. [80], D-theory is not a new set of field theories but another lattice regularization and quantization of the corresponding classical models. The main important and attractive feature is the use of discrete quantized variables and finite Hilbert spaces. However, it turns out to be necessary to formulate the theory with an additional Euclidean dimension (i.e., in $d+1$ ). In this regard dimensional reduction is a fundamental component to relate quantum link models to ordinary gauge theories. The dimensional reduction hypothesis relies on the existence of a massless phase in $d+1$, assumption that must be verified on a case-by-case basis.

The existence of a quantum link model connecting lattice gauge theories to spin (or other algebraic) theories opens the possibility of formal connections between gauge theories of high-energy physics and strongly correlated problems of condensed matter. Indeed, since there is a connection between the "Gauge World" and the "Spin World" and we exhausted the connection between the "Spin World" and the "Particle World" the consequence is that one can find isomorphisms between the "Gauge World" and the "Particle World." In this way, for instance, one may look for the exact equivalent of confinement (in gauge theories) in the particle (condensedmatter) language. Even though some of these ideas were speculated in the literature there was no formal rigorous relation established: The connection is our fundamental theorem.

\section{A. $U(1)$ Gauge Magnet}

For pedagogical purposes, it is convenient to illustrate the connection between gauge and condensed matter theories by considering the simplest $U(1)$ gauge theory, usually called gauge magnet. Gauge magnets are gauge-invariant generalizations of the Heisenberg models. The corresponding classical model has a $U(1)$ parallel transporter

$$
u_{\mathbf{j}, \mu}=e^{i \Phi_{\mathbf{j}, \mu}}
$$

associated to each link $(\mathbf{j}, \mu)$ and the action $S[u]$ is given by Eq. 181. The dagger in this case denotes complex conjugation. As it is shown by Chandrasekharan and Wiese [82], after the quantization process the $u_{\mathbf{j}, \mu}$ fields become generators of a local $s u(2)$ algebra

$$
\begin{aligned}
& U_{\mathbf{i}, \mu}=S_{\mathbf{i}, \mu}^{+}, \\
& U_{\mathbf{i}, \mu}^{\dagger}=S_{\mathbf{i}, \mu}^{-} .
\end{aligned}
$$

In other words, each link variable is replaced by a spin operator. Like in the case of quantum spin systems, this quantization can be realized with any spin representation of $S U(2)$. The simplest case corresponds to $S=1 / 2$. In this case there are two possible states for each link variable which are denoted by the two possible eigenvalues of $S_{\mathbf{i}, \mu}^{z}= \pm 1 / 2$. The Hamiltonian of the quantum $U(1)$ gauge magnet is obtained by replacing Eq. 185) into Eq. (183)

$$
H_{\mathrm{gm}}=\sum_{\mathbf{i}, \mu \neq \nu} S_{\mathbf{i}, \mu}^{-} S_{\mathbf{i}+\hat{\mu}, \nu}^{-} S_{\mathbf{i}+\hat{\nu}, \mu}^{+} S_{\mathbf{i}, \nu}^{+}
$$

and the generator of the $U(1)$ gauge symmetry is

$$
G(\mathbf{i})=\sum_{\mu}\left(S_{\mathbf{i}-\hat{\mu}, \mu}^{z}+S_{\mathbf{i}, \mu}^{z}\right) .
$$

It is natural to ask what is the equivalent particle Hamiltonian which is obtained by applying a spin-particle transformation to Eq. 186. We will only consider the simplest $S=1 / 2$ case for which Orland [83] obtained the exact solution in $2+1$ dimensions. The key observation made by Orland is that this spin model is equivalent to a gas of transversely oscillating fermionic strings. The string tension can be obtained from the exact solution and it was shown that the fermionic charges are confined. To obtain this result we need to introduce a dual 
transformation which replaces the links by sites: $(\mathbf{i}, \nu) \rightarrow \mathbf{r}$, $(\mathbf{i}, \mu) \rightarrow \mathbf{r}+\hat{x}$, and $(\mathbf{i}+\hat{\nu}, \mu) \rightarrow \mathbf{r}+\hat{y}$. This transformation is illustrated in Fig. 12 where we can see that the dual lattice is divided into a checkerboard pattern. This means that a plaquette with vertices $\mathbf{r}=\left(r_{1}, r_{2}\right), \mathbf{r}+\hat{x}, \mathbf{r}+\hat{x}+\hat{y}$, and $\mathbf{r}+\hat{y}$ is black if $r_{1}+r_{2}$ is even and white if $r_{1}+r_{2}$ is odd. The sublattice of points $\mathbf{r}$ such that $r_{1}+r_{2}$ is even will be denoted by A.

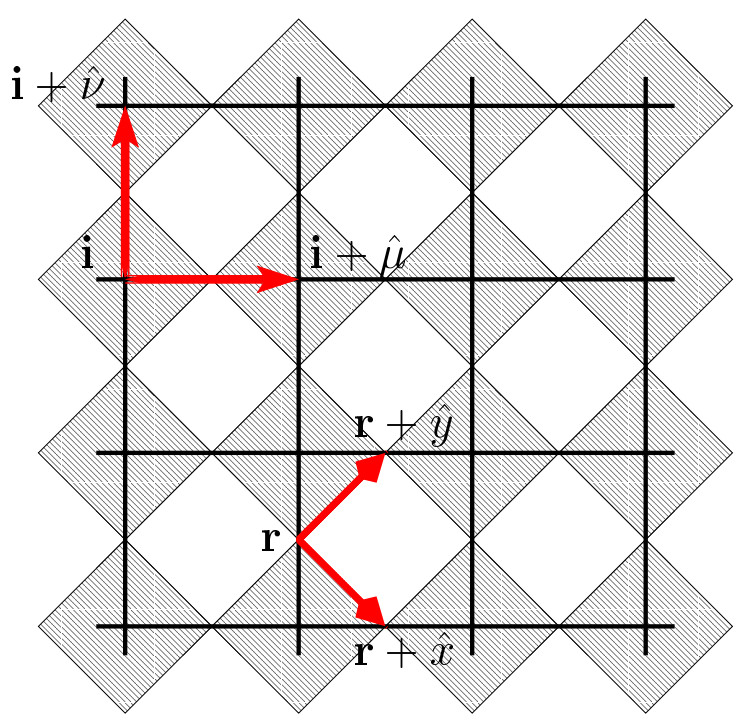

FIG. 12: Transformation from the direct square lattice, with lattice sites $\mathbf{i}$ and links $(\mathbf{i}, \mu),(\mathbf{i}, \nu)$, to the dual checkerboard lattice, with sites $\mathbf{r}$ and links $(\mathbf{r}, x)$ and $(\mathbf{r}, y)$.

The expression for $H_{\mathrm{gm}}$ after the dual transformation is

$$
\begin{aligned}
H_{\mathrm{gm}} & =\sum_{\mathbf{r} \in \mathrm{A}} S_{\mathbf{r}}^{-} S_{\mathbf{r}+\hat{y}}^{-} S_{\mathbf{r}+\hat{x}+\hat{y}}^{+} S_{\mathbf{r}+\hat{x}}^{+} \\
& +S_{\mathbf{r}+\hat{x}+\hat{y}}^{-} S_{\mathbf{r}+\hat{x}}^{-} S_{\mathbf{r}}^{+} S_{\mathbf{r}+\hat{y}}^{+} .
\end{aligned}
$$

It is clear from this expression that the black plaquettes are active and the white ones are passive. Equation 187 implies that the total charge in each white (passive) plaquette is conserved. In absence of background sources, the physical states are those which are annihilated by the 'Gauss law' operator $G(\mathbf{i})$. In the particle language, this condition means that there are two particles on each white plaquette (see for instance the state illustrated in Fig. 13.

We have seen in section $\mathrm{VC}$ that a spin $S=1 / 2$ can be transformed into a spinless hard-core anyon through the generalized JW mapping

$$
\begin{aligned}
S_{\mathbf{r}}^{+} & =\bar{a}_{\mathbf{r}}^{\dagger} K_{\mathbf{r}}^{\theta}, \\
S_{\mathbf{r}}^{-} & =\left(K_{\mathbf{r}}^{\theta}\right)^{\dagger} \bar{a}_{\mathbf{r}}, \\
S_{\mathbf{r}}^{z} & =\bar{a}_{\mathbf{r}}^{\dagger} \bar{a}_{\mathbf{r}}-\frac{1}{2} .
\end{aligned}
$$

For $\theta=0$, the anyons become hard-core bosons and for $\theta=\pi$ they are spinless fermions. By replacing these expressions into Eq. 188 we get the following Hamiltonian representing interacting hard-core anyons

$$
\begin{aligned}
H_{\mathrm{gm}} & =\sum_{\mathbf{r} \in A} \bar{a}_{\mathbf{r}} \bar{a}_{\mathbf{r}+\hat{y}} \bar{a}_{\mathbf{r}+\hat{x}+\hat{y}}^{\dagger} \bar{a}_{\mathbf{r}+\hat{x}}^{\dagger} \\
& +\bar{a}_{\mathbf{r}+\hat{x}} \bar{a}_{\mathbf{r}+\hat{x}+\hat{y}} \bar{a}_{\mathbf{r}+\hat{y}}^{\dagger} \bar{a}_{\mathbf{r}}^{\dagger} .
\end{aligned}
$$

Notice that the transmutators $K_{\mathbf{r}}^{\theta}$ and $\left(K_{\mathbf{r}}^{\theta}\right)^{\dagger}$ do not appear in the Hamiltonian. This means that $H_{\mathrm{gm}}$ is invariant under transmutation of the statistics. To physically understand the origin of this invariance we just need to realize that the dynamics imposed by $H_{\mathrm{gm}}$ only allows the motion of particles along the horizontal axis within a passive plaquette (see Fig. 13. Therefore, the original ordering of the particles is preserved and the statistics turns to be irrelevant.

The exact ground states of this model [83] correspond to parallel strings which are aligned in the $\hat{y}$ direction (see Fig. 13. The model can be exactly solved because the quantum fluctuations of each string are described by an effective onedimensional $S=1 / 2 X Y$ model [83]. Notice that this solution relates to the new paradigm in strongly correlated matter where the appearance of local inhomogeneous (stripe-like) structures seems to be a common feature of many different physical systems.

There are other possible connections between the gauge magnet and condensed matter theories which correspond to different changes of language. If each link is associated to a singlet bond state (Cooper pair), Orland [84] has shown that the $S=1 / 2$ gauge magnet is mapped onto the RokhsarKivelson model [85] (without the diagonal term) if the new 'Gauss Law': $[G(\mathbf{i})+2]|\psi\rangle=0$ is imposed. In this way we see how the formal connections between condensed matter and lattice gauge theories can be exploited to predict new physical behaviors in one or the other field.

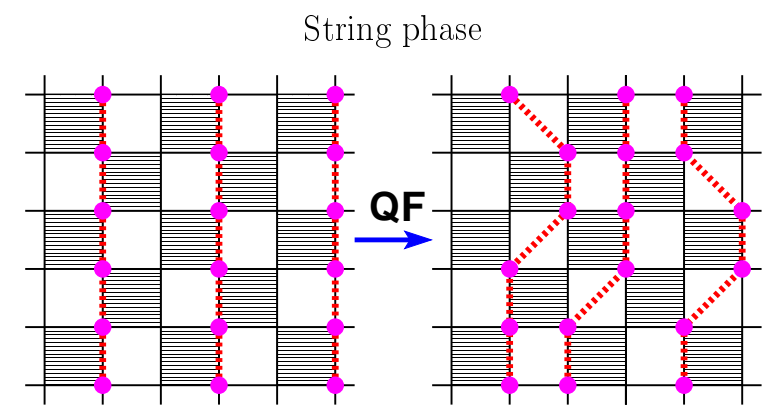

FIG. 13: Schematic picture of a ground state of the $S=1 / 2$ gauge magnet in the particle language. Quantum fluctuations $(\mathrm{QF})$ correspond to the horizontal motion of vertical pairs in the active (black) plaquettes.

\section{QUANTUM INFORMATION AND COMPUTATION}

A new challenge in information theory and computer science has recently emerged as the result of applying the fundamental laws of quantum mechanics and using the quantum 
effects to advantage. This new set of ideas comprise what is known as "Theory of Quantum Computation and Quantum Information" and has as a major objective to process information in a way that exceeds the capabilities of classical information [15]. The device that performs the manipulation of information is named quantum computer and the standard unit of information is the qubit (i.e., a two-level system). The close relationship between information processing and the physical phenomena leading to it is perhaps the most remarkable aspect of this new paradigm. Since information can be represented in many different physical forms, and easily converted from one form to another without changing its meaning, quantum information represents a new abstract archetype for information processing independent of the precise implementation of the quantum computer, only requiring at least one physical representation to be useful.

A key fundamental concept in information theory is the realization [16] that a model of computation is intimately connected to a physical system through a closed operator algebra. In other words, each physical system is associated to a certain language (e.g., spin $S=1 / 2$ ) and thus to an algebra realizing it (e.g., Pauli algebra), and that particular algebra may become a possible model of computation. An immediate consequence is that an arbitrary physical system can be simulated by another physical system (e.g., a quantum computer) whenever there exists an isomorphic mapping between the different operator algebras representing the systems [16]. A very simple example is provided in Refs. [16] and [86], where it is shown how to simulate a $1 d$ impurity Anderson model using a quantum computer based on an array of spins $S=1 / 2$. Another resource of quantum information that can help us understand complex phenomena in strongly correlated matter is the notion and measures of entanglement.

It is very clear the power that our fundamental theorem has by providing the formal connections (isomorphisms) between the different languages of nature. Therefore, the implications for quantum information and computation are rather obvious, namely that one can identify quantum resources and define convenient models of computation, or imitate an arbitrary quantum phenomena with a given quantum computer given the appropriate dictionaries to translate nature's language to the machine language. In this way, one can recognize three complementary areas where the concepts of language and dictionaries developed in the present manuscript are of particular relevance, areas we will expand on in the following.

\section{Models of computation and quantum resources}

A model of computation consists of an algebra of operators, a set of controllable Hamiltonians and measurable observables, and an initial state of the physical system. The set of controllable Hamiltonians must be universal in the sense that any unitary operation can be performed with such a set. The standard model of computation is a particular example which uses the Pauli operator algebra and the qubit as basic unit. Another possible model is the fermion model [16] which is isomorphically related to the standard one through the JW mapping and, thus, is equivalent. The main point is that the choice of model depends upon the nature of the available physical resources and their quantum control. For example, in a liquid NMR quantum computer the nuclear spins of the molecules $(S=1 / 2)$ are the units which can be controlled and the standard model is the appropriate one.

The control of quantum mechanical systems is hampered by quantum noise and decoherence and, therefore, identifying static quantum resources for information processing is a great challenge. Given a physical information processing device there will be a language which will the most natural one for the elementary static resources of the device. For instance, if we could control ${ }^{4} \mathrm{He}$ atoms at the quantum level it would be natural to consider a hard-core boson model of computation. The importance of our fundamental theorem is that once one identifies the best quantum resources it allows us to build the model of computation accordingly.

Simulation of physical phenomena

Physical phenomena can be simulated or imitated by a quantum network [87] with the help of a quantum computer. Imitation is realized through a quantum algorithm which consists of a quantum network with a means to repeat blocks of instructions. A quantum network is defined by a sequence of universal gates (unitary operations), applied to the system for the purpose of information processing, and measurements in a fixed temporal order. The measurement operation is mostly needed to classically access information about the state of the system. Every matrix which represents a reversible operation on quantum states can be expressed as a product of the one and two-qubit gates, and the minimum set needed to represent any such matrices is called a universal set of gates.

When trying to simulate a problem using quantum information processing, an important issue is to determine how many physical resources are needed for the solution. The main resources are quantum space, the number of qubits needed, and quantum time, the number of quantum gates required. The accounting of algorithmic resources forms the foundations of quantum complexity theory. One of the objectives in quantum information theory is to accomplish imitation efficiently, i.e, with polynomial complexity, and the hope is that quantum imitation is more efficient (i.e., needs less resources) than classical imitation. There are examples that support such hope (e.g., fermion simulations with polynomially bounded statistical errors [16, 87]), although there is no general proof that indicates the superiority of quantum over classical imitations, regarding efficiency. Indeed, there is, so far, no efficient quantum algorithm that can determine the ground state (or, in general, the spectrum) of a given Hermitian operator [87], despite occasional claims. It is known that the ability to resolve this question leads to efficient algorithms for NPcomplete problems like the traveling salesman conundrum. 
A very important observation, in connection with the notion of efficiency, is a corollary of our fundamental theorem: Given two languages, the generators of one of them can be written as a polynomial function, with polynomial complexity in the number of modes or resources, of the generators of the other and vice versa. This result implies that the important algorithmic step of translation from the language of the system to be imitated to the machine language does not change the complexity of the quantum space and time.

Certainly, a general purpose quantum computer is not the only device that allows simulation of physical phenomena in nature (with its many languages). Imitation can also be achieved in a conceptually different manner using a quantum simulator. The main distinction is the lack of universality of the latter. An example of a quantum simulator is an optical lattice [88] which is specifically designed to imitate a given physical Hamiltonian and where there is limited quantum control. The possibility of control and tunability of the interactions of the elementary constituents offers the potential to design new states of matter [60, 88]. This is of particular relevance in strongly correlated matter where these quantum simulators furnish the benchmark to test theories and approximations [60]. Again, the importance of the languages and dictionaries developed in this manuscript is clear and concrete.

Quantum information measures

Entanglement, a word that Schrödinger coined to distinguish quantum from classical mechanics, is that bizarre feature of composite quantum systems that led to so much controversy in the past (like quantum nonlocality in the Einstein-Podolsky-Rosen gedanken experiment). Essentially, entanglement is a quantum property whereby a pure state of a composite quantum system may cease to be determined by the states of its constituent subsystems. Entangled pure states are those that have mixed subsystem states. An independent concept is the notion of separability which refers to the property of a quantum state of a composite system of being able to be written as a direct product of states of its component subsystems. The theory of entanglement, and its generalizations, is currently under development [15, 89]. In Ref. [89] entanglement is viewed as an observer-dependent concept, whose properties are determined by the expectations of a distinguished subspace of observables of the system of interest (experimental access), without reference to a preferred subsystem decomposition. The standard notion of entanglement is recovered when these means are limited to local observables acting on subsystems. A tremendous effort is put in trying to understand the properties of entanglement that can be used as a resource in quantum information.

In a sense, that must be defined more precisely [89], the notion of entanglement is a relative of the notion of quantum correlations and a very relevant question is whether one can construct useful measures of entanglement to understand the emergence of complex phenomena in strongly correlated matter. A quantum phase transition involves a qualitative change in the correlations of the ground state of the system as a result of tuning parameters of its Hamiltonian. In some cases an order parameter is associated to the transition, in others a topological order. It is intuitively expected that this change is also associated to a change in the nature of entanglement. Therefore, quantifying and classifying entanglement is very important to characterize a quantum phase transition: Can measures of entanglement distinguish between broken and non-broken [2] symmetry phase transitions?

\section{SUMMARY}

We have introduced an algebraic framework for interacting quantum systems to study complex phenomena characterized by the coexistence and competition of various ordered states. We argued that symmetry, and topology, are key guiding principles behind such complex emergent behavior. Emphasis has been made in developing a systematic mathematical structure that allows one to attack these problems within a single unifying approach.

The core result of the paper, from which all other results follow, is the proof of a fundamental theorem that permits to connect the various operator languages used in the description of the properties of physical systems. This theorem together with the notion of transmutation of statistics provide the tools necessary to unifying the quantum description of matter. To formalize this unification we needed to rigorously define the concepts of language and dictionary: To model a particular physical phenomena we commonly identify the main degrees of freedom of the problem and associate to them certain operators. One can furnish to the resulting set of operators (that we call language) with an algebraic structure and ask whether two different languages have something in common. The fundamental theorem tells us that two languages can be connected whenever the dimension of their local Hilbert spaces are equal. We expanded the notion of local Hilbert space to embrace different Hilbert space decompositions (we saw, for instance, how to map the Hilbert space of a bond to a site). The resulting one-to-one language mappings we named dictionaries (a traditional example of which is the Jordan-Wigner mapping). In the course of the presentation we showed, through example, many different dictionaries relating diverse operator languages. In this way we defined universality of behavior as an equivalence relation between seemingly different physical phenomena which share exactly the same underlying mathematical structure as a result of one-to-one language mappings (for example, the spin nematic order and Bose-Einstein condensation of flavored hardcore bosons). Out of the many languages one can use to describe a given physical problem there is a class, we named hierarchical language, which has the advantage that any local operator can be expressed as a linear combination of its gen- 
erators. In this way, hierarchical languages provide the tools necessary to classify order parameters.

A new, formally developed, notion is the idea of emergent symmetry, i.e., the fact that new symmetries not realized in the Hamiltonian describing the system can emerge at low energies. From a broader perspective, an emergent symmetry is a non-local transformation which commutes with the system Hamiltonian and becomes local when restricted to a low-energy subspace of the Hilbert space. In some instances like the quasi-exactly solvable problems, to which the $1 d t$ $J_{z}$ model belongs [6], or the family of spin Hamiltonians for which the ground state is a product of spin singlets (e.g., the Majumdar-Ghosh model), the low-energy effective theory can be derived in an exact way and the emergent symmetry is exact. In other cases, the emergent symmetry is approximate, however, it still provides a guiding principle to identify the nature of the ground state and its low-energy excitations. In the same way that rigidity is related to a broken symmetry phenomenon, localization (in real or any other space) is connected to the emergence of a gauge symmetry. Indeed, the metal-insulator transition may be interpreted as a discontinous change in the group of emergent symmetries characterizing the two phases.

Figure 14 summarizes the spirit and fundamental concepts that emerge from our fundamental theorem.

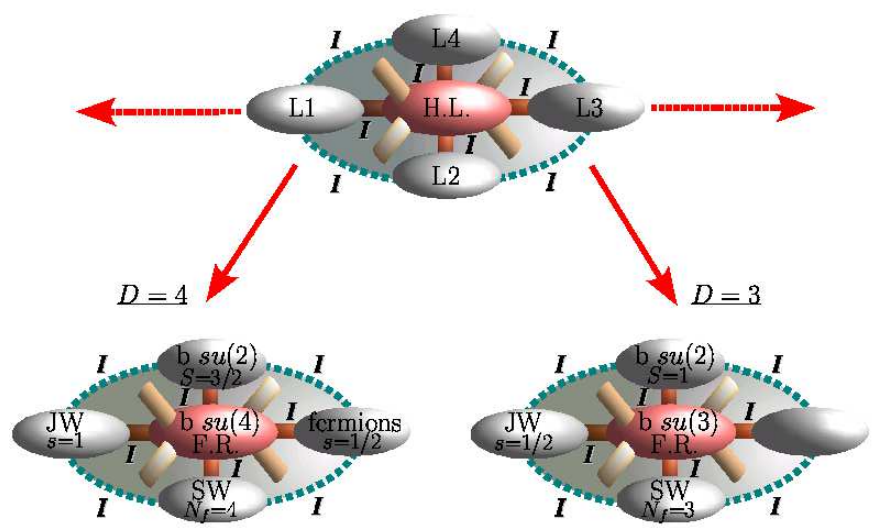

FIG. 14: Unified Framework. For each $D$ there is more than one diagram with a different hierarchical language (H.L.). I indicates that there is an isomorphic mapping between two languages, e.g., L1 and L2.
There are several reasons for our algebraic framework to constitute a powerful method to study complex phenomena in interacting quantum systems. Most importantly: To connect seemingly unrelated physical phenomena (e.g., high- $T_{c}$ or heavy fermions and quantum spin theories); identify the general symmetry principles behind complex phase diagrams; unveil hidden symmetries (and associated order parameters) to explore new states of matter; obtain exact solutions of relevant physical models that display complex ordering at certain points in Hamiltonian space; and find new approximations which do not favor any of the competing interactions. The power of the present approach is reflected in the unlimited number of potential applications where they could be used, and ranges from condensed matter and statistical mechanics to lattice gauge theories and quantum information and computation. We hope and envision this formalism will find future applications in contexts different than the ones we have developed here.
Acknowledgments

We thank J.E. Gubernatis, L. Gurvits, W. Lee, and B.S. Shastry for useful discussions. This work was sponsored by the US DOE under contract W-7405-ENG-36.
[1] P.W. Anderson, Science 177, 393 (1972).

[2] See, for example, X.-G. Wen, Phys. Rev. B 65, 165113 (2002).

[3] P. Jordan and E. Wigner, Z. Phys. 47, 631 (1928).

[4] T. Matsubara and H. Matsuda, Prog. Theor. Phys. 16, 569 (1956).

[5] A.G. Ushveridze, Quasi-Exactly Solvable Models in Quantum Mechanics (IOP, Bristol, 1994).

[6] C.D. Batista and G. Ortiz, Phys. Rev. Lett. 85, 4755 (2000).
[7] L.D. Landau and E.M. Lifshitz, Statistical Physics (Butterworth-Heinemann, Oxford, 2001).

[8] P.W. Anderson, Basic Notions of Condensed Matter Physics (Addison-Wesley, Redwood City, 1992).

[9] C.K. Majumdar and D.K. Gosh, J. Math. Phys. 10, 1388 (1969); 10, 1399 (1969); C.K. Majumdar, J. Phys. C 3, 911 (1970).

[10] B.S. Shastry and B. Sutherland, Physica B \& C 108, 1069 (1981). 
[11] B.S. Shastry and B. Sutherland, Phys. Rev Lett. 47, 964 (1981).

[12] B. Sutherland and B.S. Shastry, J. Stat. Phys. 33, 477 (1983).

[13] H.Q. Lin, J.L. Shen, and H.Y. Shik, cond-mat/0109027

[14] N. Papanicolau, Phys. Lett. A 116, 89 (1986); Nucl. Phys. 305, 367 (1988).

[15] See for example M.A. Nielsen and I.L. Chuang, Quantum Computation and Information (Cambridge, Cambridge, 2000).

[16] G. Ortiz, J.E. Gubernatis, E. Knill, and R. Laflamme, Phys. Rev. A 64, 22319 (2001).

[17] See for example A. Bohm, Quantum Mechanics: Foundations and Applications (Springer-Verlag, New York, 1993).

[18] A monoid can also be defined as a semigroup (M, $\square$ ) with an element that is the unit for $\square$, i.e., 1 .

[19] Commutators (Anticommutators) will be represented by square (curly) brackets, i.e., $[A, B]=A B-B A(\{A, B\}=A B+$ $B A$ ). Sometimes we will invoke the generalized deformed commutator defined as: $[A, B]_{\theta}=A B-\exp [i \theta] B A$. We may also use $[A, B]_{ \pm}=A B \pm B A$. Canonical commutation (anticommutation) relations for the corresponding creation and annihilation operators are generically defined as

$$
\begin{array}{r}
{\left[b_{\mathbf{j} \alpha}, b_{\mathbf{j} \beta}^{\dagger}\right]=\left\langle\varphi_{\alpha} \mid \varphi_{\beta}\right\rangle,\left[b_{\mathbf{j} \alpha}, b_{\mathbf{j} \beta}\right]=0} \\
\left(\left\{c_{\mathbf{j} \alpha}, c_{\mathbf{j} \beta}^{\dagger}\right\}=\left\langle\varphi_{\alpha} \mid \varphi_{\beta}\right\rangle,\left\{c_{\mathbf{j} \alpha}, c_{\mathbf{j} \beta}\right\}=0\right),
\end{array}
$$

where the inner product $\left\langle\varphi_{\alpha} \mid \varphi_{\beta}\right\rangle$ between elements of a singleparticle basis $(\alpha, \beta \in[1, D])$ is defined on $\mathcal{H}_{\mathbf{j}}$. For the sake of clarity and with no loss of generality, we will always assume that the single-particle basis $\left\{\varphi_{\alpha}\right\}$ is orthonormal, i.e., $\left\langle\varphi_{\alpha} \mid \varphi_{\beta}\right\rangle=\delta_{\alpha \beta}$, but, of course, this condition can be relaxed.

[20] N. Jacobson, Basic Algebra I (W.H. Freeman and Company, New York, 1985), and Basic Algebra II (W.H. Freeman and Company, New York, 1989).

[21] For a $d$-dimensional lattice with $N_{s}$ sites, the operator $S_{\mathbf{j}}^{\mu}$ is defined in terms of a Kronecker product $\otimes$ as

$$
S_{\mathbf{j}}^{\mu}=\mathbb{1} \otimes \mathbb{1} \otimes \cdots \otimes \underbrace{S^{\mu}}_{\mathbf{j}^{t h}} \otimes \cdots \otimes \mathbb{1},
$$

where $\mathbb{1}$ is the $D \times D$ unit matrix, $D=2 S+1$, and $S^{\mu}$ is a spin- $S$ operator. Thus $S_{\mathbf{j}}^{\mu}$ admits a matrix representation of dimension $D^{N_{s}} \times D^{N_{s}}$.

[22] C.D. Batista and G. Ortiz, Phys. Rev. Lett. 86, 1082 (2001).

[23] The $S O(5)$ theory of Zhang [24] constitutes a particular example of a unified (high-symmetry) order parameter used to describe the coexistence of more than one phase (in this case superconductivity and antiferromagnetism). However, contrary to the philosophy of this paper, this description relies on the assumption of an $S O(5)$ symmetry of the low-energy effective theory, assumption whose validity is unclear. According to Zhang [24], this high-symmetry effective theory can be obtained by applying the renormalization group.

[24] S-C. Zhang, Science 275, 1089 (1997).

[25] B. Abdullaev, C.D. Batista, and G. Ortiz (unpublished).

[26] J.F. Cornwell, Group Theory in Physics (Academic Press, London, 1989).

[27] If the system has long-range interactions the dimension of the lattice becomes irrelevant, since any $d$-dimensional system can be described in a lower dimensional lattice at the cost of including long-range interactions.

[28] G. Ortiz and C.D. Batista in Recent Progress in Many-Body Theories, Eds. Raymond F Bishop, Tobias Brandes, Klaus A Gernoth, Niels R Walet, and Yang Xian (World Scientific, Singapore, 2002), p. 425; Int. J. of Modern Phys. B 17, 5413 (2003).
[29] E. Fradkin, Phys. Rev. Lett. 63, 322 (1989); D. Eliezer and G.W. Semenoff, Phys. Lett. B 286, 118 (1992).

[30] Y.R. Wang, Phys. Rev. B 43, 3786 (1991).

[31] L. Huerta and J. Zanelli, Phys. Rev. Lett. 71, 3622 (1993).

[32] S. Wang, Phys. Rev. E 51, 1004 (1995).

[33] Suppose that we want to establish a particular ordering for twoflavored particles $(\alpha=\uparrow, \downarrow)$ in a finite $N_{s}=N_{x} \times N_{y} 2 d$ lattice. A possible ordering from $(\mathbf{j}, \alpha)$ to an ordered set of integers I could be

$$
\mathrm{I}=j_{1}+\left(j_{2}-1\right) N_{x}+\left(\frac{1}{2}-\sigma\right) N_{x} N_{y}
$$

where $\sigma=\frac{1}{2}\left(-\frac{1}{2}\right)$ for $\alpha=\uparrow(\downarrow)$.

[34] H.S. Green, Phys. Rev. 90, 270 (1953).

[35] C.D. Batista and G. Ortiz, in Condensed Matter Theories, vol. 16, edited by Susana Hernandez and W. John Clark (Nova Science Publishers, Inc., Huntington, New York, (2001)) pp. 1.

[36] K. Huang, Statistical Mechanics (Wiley, New York, 1963), Chap. 16.

[37] These isomorphisms represent useful dictionaries translating a spin language $S$ into itinerant quantum particle languages with effective spin $s=S-1 / 2$.

[38] G. Gentile, Nuovo Cim. 17, 493 (1940).

[39] See for example A. Khare, Fractional Statistics and Quantum Theory (World Scientific, Singapore, 1997).

[40] F.D.M. Haldane, Phys. Rev. Lett. 67, 937 (1991).

[41] Fermions correspond to the case $p=2$, while for bosons $p$ is an arbitrary integer number larger than 2 .

[42] O.W. Greenberg, Phys. Rev. Lett. 13, 598 (1964).

[43] H.S. Green, Prog. Theor. Phys. 47, 1400 (1972).

[44] S. Kamefuchi and Y. Takahashi, Nucl. Phys. 36, 177 (1962); C. Ryan and E.C.G. Sudarshan, ibid 47, 207 (1963); K. Kademova, Nucl. Phys. B 15, 350 (1970); A.J. Bracken and H.S. Green, Nuovo Cim. 9, 349 (1972).

[45] Care should be exercised regarding the boundary conditions of the model. Keeping this warning in mind we will omit any reference to boundary conditions in the following.

[46] C.D. Batista, G. Ortiz, and J.E. Gubernatis, Phys. Rev. B 65, 180402(R) (2002).

[47] R.J. Baxter, Exactly Solved Models in Statistical Mechanics (Academic Press, London, 1990).

[48] C.K. Lai, J. Math. Phys. 15, 1675 (1974); B. Sutherland, Phys. Rev. B 12, 3795 (1975).

[49] L.A. Takhtajan, Phys. Lett. A 87, 479 (1982); H.M. Babujian, Phys. Lett. A 90, 479 (1982); Nucl. Phys. B 215, 317 (1983).

[50] I. Affleck, T. Kennedy, E.H. Lieb, and H. Tasaki, Phys. Rev. Lett. 59799 (1987).

[51] A. Klümper, Europhys. Lett. 9, 815 (1989).

[52] L. Arrachea and A.A. Aligia, Phys. Rev. Lett. 732240 (1994).

[53] B. Sutherland, Phys. Rev. B 62, 11499 (2000).

[54] D. C. Mattis and S. B. Nam, J. Math. Phys. 13, 1185 (1972).

[55] C.D. Batista, G. Ortiz, and B.S. Shastry (unpublished).

[56] E.H. Lieb and F.Y. Wu, Phys. Rev. Lett. 20, 1443 (1968).

[57] A. Auerbach, Interacting Electrons and Quantum Magnetism (Springer-Verlag, New York, 1994).

[58] P.W. Anderson, Phys. Rev. 110, 827 (1958); 112, 1900 (1958).

[59] Other representations with different particle statistics are possible; for example, we could have used a hard-core fermion representation [22]. In such a case, the resulting Hamiltonian would be similar in form to Eq. 128 with a kinetic energy term modified to include a non-local gauge field.

[60] G. Ortiz and C.D. Batista, Phys. Rev. B 67, 134301 (2003).

[61] P.A. Martin, Nuovo Cimento 68B, 302 (1982).

[62] B. Kumar, Phys. Rev. B 66, 024406 (2002). 
[63] C.D. Batista and B.S. Shastry, Phys. Rev. Lett. 91, 116401 (2003).

[64] Ph. Nozières, Theory of Interacting Fermi Systems (AddisonWesley, Reading, 1997).

[65] Z.N.C. Ha, Quantum Many-Body Systems in One Dimension (World Scientific, Singapore, 1996).

[66] After Friedrich August Kekulé, a German chemist, who solely on intuition proposed the known resonant structure of benzene in 1872, before quantum mechanics was born. It was Linus Pauling who formally developed the resonance theory in 1931.

[67] J. Sólyom, Phys. Rev. B 36, 8642 (1987).

[68] J. B. Parkinson, J. Phys. C 21, 3793 (1988).

[69] M. N. Barber and M. T. Batchelor, Phys. Rev. B 40, 4621 (1989).

[70] A. Klumper, Europhys Lett. 9, 815 (1989); J. Phys. A 23, 809 (1990).

[71] A.V. Chubukov, J. Phys. Condens. Matter 2, 1593 (1990).

[72] A.V. Chubukov, Phys. Rev. B 43, 3337 (1991).

[73] G. Fáth and J. Sólyom, Phys. Rev. B 44, 11836 (1991).

[74] G. Fáth and J. Sólyom, Phys. Rev. B 47, 872 (1993).

[75] T. Xiang and G. A. Gehring, Phys. Rev. B 48, 303 (1993).

[76] Y. Xian, J. Phys. Condens. Matter 5, 7489 (1993).
[77] Y. Xian, J. Phys. Lett. A 183, 437 (1993).

[78] G. Fáth and J. Sólyom, Phys. Rev. B 51, 3620 (1995).

[79] See, for example, I. Montvay and G. Münster, Quantum Fields on a Lattice (Cambridge, Cambridge, 1994).

[80] U.-J. Wiese, Nucl. Phys. B 73, 146 (1999) and references therein.

[81] D. Horn, Phys. Lett. B 100, 149 (1981).

[82] S. Chandrasekharan and U.-J. Wiese, hep-lat/9609042

[83] P. Orland, Nucl. Phys. B 372, 635 (1992).

[84] P. Orland, Phys. Rev. B 49, 3423 (1994).

[85] D. S. Rokhsar and S. A. Kivelson, Phys. Rev Lett. 61, 2376 (1988).

[86] G. Ortiz, E. Knill, and J.E. Gubernatis, Nuc. Phys. B 106, 151 (2002).

[87] R. Somma, G. Ortiz, J.E. Gubernatis, R. Laflamme, and E. Knill, Phys. Rev. A 65, 42323 (2002); R. Somma, G. Ortiz, E. Knill, and J.E. Gubernatis, Int. J. of Quant. Inf. 1, 189 (2003).

[88] M. Greiner et al., Nature 415, 39 (2002).

[89] H. Barnum, E. Knill, G. Ortiz, and L. Viola, Phys. Rev. A 68, 032308 (2003). See also quantum-ph/0305023 